\title{
Measurement of the Top Quark Mass by Dynamical Likelihood Method using the Lepton plus Jets Events in 1.96 TeV Proton-Antiproton Collisions
}

\author{
A thesis presented \\ by \\ Kohei Yorita
}

To

The Department of Physics

in partial fulfillment of the requirements

for the degree of Doctor of Philosophy

in the subject of Physics

Waseda University

Tokyo, Japan

March 2005 



\section{Preface}

We have measured the top quark mass with the dynamical likelihood method (DLM) using the CDF II detector at the Fermilab Tevatron. The Tevatron produces top and anti-top pairs in $p \bar{p}$ collisions at a center-of-mass energy of $1.96 \mathrm{TeV}$. The data sample used in this paper was accumulated from March 2002 through August 2003 which corresponds to an integrated luminosity of $162 \mathrm{pb}^{-1}$. We use the $t \bar{t}$ candidates in the "lepton+jets" decay channel with at least one $b$ tagged jet requirement. In DLM, the likelihood is defined as the differential cross section as a function of $\mathrm{M}_{\text {top }}$ per unit phase space volume of the final partons, multiplied by the transfer function from jets to parton quantities. The method takes into account all possible jet combinations in an event and the likelihood is multiplied event by event to derive the top quark mass by the maximum likelihood method. Using $22 t \bar{t}$ candidates observed in the data with 4.2 events expected from background, we measured the top quark mass to be $177.8_{-5.0}^{+4.5}$ (stat.) \pm 6.2 (syst.) $\mathrm{GeV} / \mathrm{c}^{2}$. 
PREFACE 


\section{Acknowledgments}

I would like to thank my supervisor Kunitaka Kondo for his guidance, teaching and support during my time as a graduate student. His boundless enthusiasm for physics has always inspired me and his words have been sources of wisdom in matters of both physics and life as a physicist. I learned lots of things from him. I would also like to thank Shinhong Kim and other stuff members of CDF Japan group for providing me with the opportunity to work at Fermilab. Also I would like to thank all members at Waseda University, in particular Koji Ebina for his friendliness.

Needless to say, this dissertation could be made possible by the efforts of the CDF collaboration and the Fermilab staff. So I would like to thank all of the people who have proposed, designed, constructed, developed and maintained the accelerators, the CDF detector and analysis environment for their strong and excellent supports, operations and efforts.

In my early years as a graduate student, I had a great opportunity to work for the scintillation counters for muon detection with especially Giovanni Pauletta and Warren Bowman. I want to thank them for their kindness and teaching me very important aspects of the experiments through rebuilding and testing hundreds of counters and installing them into the CDF detector.

My deep appreciation is due to Evelyn Thomson, Ken Bloom, Jason Nielsen, Young-Kee Kim, Igor Volobouev, Jaco Konigsberg, Patrizia Azzi and all other members in CDF Lepton+Jets, TopMass, Top, and Jet Energy Correction Group for cooperation, encouragement and careful review of my analysis. I would also like to thank Kaori Maeshima for helping me work for Consumer Online Monitoring system.

I would like to express my great appreciation to Carol Piccolo, Kyoko Kunori and Kazuko Kumashiro for taking care of administrative issues through their secretary works.

I would like to greatly thank Rene Padilla and Chivas Makaroplos whom I could feel like my parents in the United States. I have appreciated their kindness. My special thanks also go to Soon Yung Jun, Sungwon Lee and Yeon Sei Chung for their continuous encouragements 
and teaching me an importance of "sense of human". I would also like to thank Takasumi Maruyama, Masashi Tanaka and Tomonobu Tomura for valuable discussion, proper guidance and most importantly lots of good Sake.

This work was supported by the U.S. Department of Energy and National Science Foundation; the Italian Istituto Nazionale di Fisica Nucleare; the Ministry of Education, Culture, Sports, Science and Technology of Japan; the Natural Sciences and Engineering Research Council of Canada; the National Science Council of the Republic of China, the Swiss National Science Foundation; the A.P. Sloan Foundation; the Bundesministerium für Bildung und Forschung, Germany; the Korean Science and Engineering Foundation and the Korean Research Foundation; the Particle Physics and Astronomy Research Council and the Royal Society, UK; the Russian Foundation for Basic Research; the Comision Interministerial de Ciencia y Tecnologia, Spain; and in part by the European Community's Human Potential Programme under contract HPRN-CT-20002, Probe for New Physics.

Finally, I would like to thank my family for their support. Your understanding has been amazing. Thank you very much. 


\section{Contents}

$\begin{array}{ll}\text { Preface } & \text { i }\end{array}$

$\begin{array}{ll}\text { Acknowledgments } & \text { iii }\end{array}$

$\begin{array}{ll}\text { List of Figures } & \text { ix }\end{array}$

List of Tables $\quad$ xv

1 Introduction $\quad 1$

1.1 The Standard Model . . . . . . . . . . . . . . . . . . 2

1.2 Top Quark Physics . . . . . . . . . . . . . . . . . 5

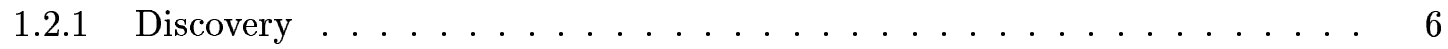

1.2 .2 Production and Decay . . . . . . . . . . . . . . 6

1.2.3 $t \bar{t}$ Cross Section Measurements . . . . . . . . . . . . . . 9

1.3 The Mass of Top Quark . . . . . . . . . . . . . . . . 11

1.3.1 Results from the Tevatron in Run I and Run II . . . . . . . . . . . . 13

1.3.2 Relation and Constraint on the Higgs Boson Mass . . . . . . . . . . . 14

2 Experimental Design $\quad 19$

2.1 The Accelerator Complex . . . . . . . . . . . . . . . . . 19

2.1 .1 Proton production and boosting . . . . . . . . . . 22

2.1 .2 Main Injector . . . . . . . . . . . . . . . . . . 22

2.1 .3 Antiproton production . . . . . . . . . . . . . 23

2.1 .4 Recycler Ring . . . . . . . . . . . . . . . . 23

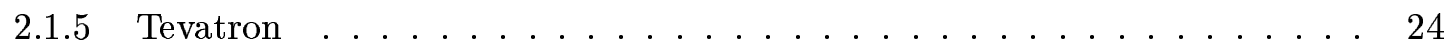

2.1.6 Beam monitors . . . . . . . . . . . . . . . . 24 
2.2 The CDF Detector . . . . . . . . . . . . . . . 25

2.3 Tracking system . . . . . . . . . . . . . . . 26

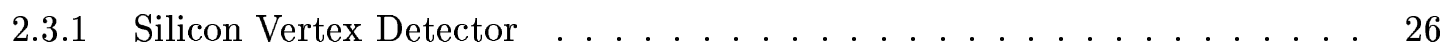

2.3.2 Central Outer Tracker . . . . . . . . . . . . . . . 27

2.3 .3 Time of flight . . . . . . . . . . . . . . 28

2.3 .4 Magnet . . . . . . . . . . . . . . . . . 28

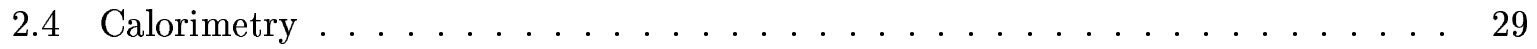

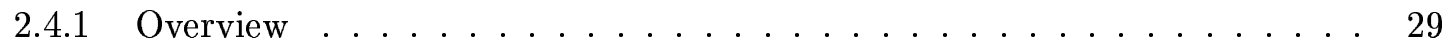

2.4 .2 Central calorimeter . . . . . . . . . . . . . . . . 29

2.4 .3 Plug calorimeter upgrade . . . . . . . . . . . . . 31

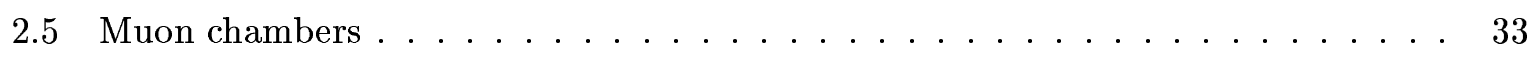

2.5.1 Central muon detectors . . . . . . . . . . . . 34

2.5.2 Intermediate muon detectors $\ldots \ldots \ldots \ldots \ldots$

2.6 Data Acquisition and Trigger . . . . . . . . . . . . . 37

2.6 .1 Level 1 trigger $\ldots \ldots \ldots \ldots \ldots \ldots \ldots \ldots \ldots$

2.6 .2 Level 2 trigger $\ldots \ldots \ldots \ldots \ldots \ldots \ldots \ldots$

2.6 .3 Level 3 trigger . . . . . . . . . . . . . . . . 39

2.6 .4 Online Monitoring . . . . . . . . . . . . . . . 40

3 Particle Identifications $\quad 43$

3.1 Lepton Identification . . . . . . . . . . . . . . . . . . . . 43

3.2 Jet Identification and Corrections . . . . . . . . . . . . . . . . . . 44

$3.3 \quad b$ Jet Tagging using Secondary Vertex $\ldots \ldots \ldots$. . . . . . . . . . . 46

3.4 Missing Transverse Energy $\boldsymbol{t}_{T} \ldots \ldots \ldots \ldots \ldots$. . . . . . . . . 48

4 Data Samples and Event Selection $\quad 51$

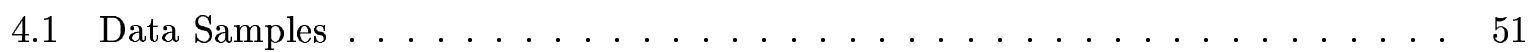

4.2 Monte Carlo Programs . . . . . . . . . . . . . . . . . . 52

4.3 Event Selection . . . . . . . . . . . . . . . . . 52

$5 \quad$ Measurements of $t \bar{t}$ Production Cross Section $\quad 57$

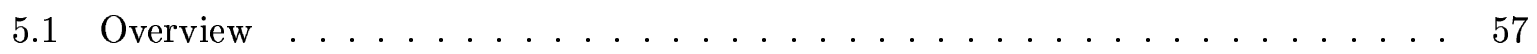

$5.2 t \bar{t}$ Production Cross Section . . . . . . . . . . . . . . 58

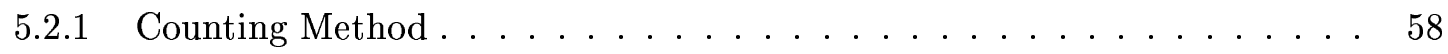


5.3 Background Estimation used for this analysis $\ldots \ldots \ldots$. . . . . . . 67

6 Measurement of Top Quark Mass $\quad 69$

6.1 Analysis Overview . . . . . . . . . . . . . . . . . . 69

6.2 Dynamical Likelihood Method . . . . . . . . . . . . . . 70

6.2 .1 Differential cross section . . . . . . . . . . . . . 70

6.2 .2 Transfer functions for observables . . . . . . . . . . . 71

6.2 .3 Resonances . . . . . . . . . . . . . . . . . . 72

6.2.4 Reconstruction of parton kinematics . . . . . . . . . . . 73

6.2 .5 Likelihood for an event . . . . . . . . . . . . . 73

6.2.6 Matrix Element Calculation in the lepton+jets channel . . . . . . . . 74

6.3 Transfer Function . . . . . . . . . . . . . . . . . . . 76

6.3.1 Definition and Performance . . . . . . . . . . . . 76

6.3.2 Missing Transverse Energy Treatments . . . . . . . . . . . . . 81

6.4 Performances of the Method . . . . . . . . . . . . . . . 81

6.4.1 Tests using Signal Monte Carlo Simulation . . . . . . . . . . . . 81

6.4 .2 Backgrounds Effect on the Likelihood . . . . . . . . . . 83

6.4.3 The mapping function to extract the top quark mass . . . . . . . . 87

6.4.4 Sanity check: mass pull distribution after using the mapping functions . . 88

6.4 .5 Blind Test Results . . . . . . . . . . . . . . . . . . 91

$\begin{array}{llr}7 & \text { Results } & 93\end{array}$

7.1 Extracting Top Quark Mass . . . . . . . . . . . . . . . . 93

7.2 The Statistical Uncertainty $\ldots \ldots \ldots \ldots$. . . . . . . . . . . . 97

8 Systematic Uncertainty $\quad 99$

8.1 Jet energy scale . . . . . . . . . . . . . . . . . . . . . . . . 99

8.2 Initial and final state hard radiation (ISR, FSR) $\ldots \ldots \ldots$

8.3 Parton Distribution Function $(\mathrm{PDF}) \ldots \ldots \ldots \ldots$

8.4 Next-to-Leading Order uncertainty . . . . . . . . . . . . . . . 103

8.5 Monte Carlo modeling . . . . . . . . . . . . . . . . . 104

8.5.1 Jet energy resolution differences . . . . . . . . . . . . . . 104

8.5.2 Unclustered energy for $\boldsymbol{E}_{T} \ldots \ldots \ldots \ldots$. . . . . . . . . . 104

8.6 Background modeling and fraction . . . . . . . . . . . . . . 104 
8.7 Monte Carlo generators . . . . . . . . . . . . . . . . . 104

8.8 Summary of systematic uncertainties . . . . . . . . . . . . 105

9 Cross Checks $\quad 107$

9.1 Event by event comparisons . . . . . . . . . . . . . 107

$\begin{array}{ll}10 \text { Conclusions } & 111\end{array}$

$\begin{array}{ll}\text { Bibliography } & 113\end{array}$

$\begin{array}{ll}\text { Publication List } & 117\end{array}$ 


\section{List of Figures}

1.1 Comparison of masses of Standard Model quarks [6] . . . . . . . . . . . . 4

1.2 The lowest order diagrams for $t \bar{t}$ production at the Tevatron . . . . . . . . . 7

1.3 Comparison of $t \bar{t}$ production cross section between Run $\mathrm{I}(\sqrt{s}=1.8 \mathrm{TeV})$ and Run II $(\sqrt{s}=1.96 \mathrm{TeV})$ for the experimental results (points) and the Standard Model calculation by theory (blue band). . . . . . . . . . . 8

1.4 The tree-level Feynman diagram for $t \bar{t}$ production by $q \bar{q}$ annihilation and its standard model decay chain. . . . . . . . . . . . . . . . . 10

1.5 The Feynman diagram for $W+$ multijet production which is one of the backgrounds to the top signal. . . . . . . . . . . . . . . . . . 11

1.6 Comparison of measured values of $t \bar{t}$ production cross section $\left(\sigma_{t \bar{t}}\right)$ at CDF Run II. 12

1.7 Summary of the top quark mass measured by using Run I Tevatron data. di-l, $1+\mathrm{j}$ and all-j stand for dilepton, lepton+jets and all-hadronic channels respectively. 13

1.8 Summary of the top quark mass measured by using Run II Tevatron data obtained so far. Three results with dilepton channel, two results with lepton+jets channel and the world average are shown. Note that the result of this analysis is not shown in the plot. . . . . . . . . . . . . . . 15

1.9 Contribution of self-coupling loops to higher order quantum corrections. . . . . 16

1.10 The comparison of the indirect measurements of $M_{W}$ and $M_{t o p}$ (solid contour) and the direct measurements ( $p \bar{p}$ colliders and LEP-II data) (dashed contour). In both cases the $68 \%$ C.L. contours are plotted. Also shown in the Standard Model relationship for the masses as a function of the Higgs mass. The arrow labeled $\Delta \alpha$ shows the variation of this relation if $\alpha\left(M_{Z}^{2}\right)$ is changed by one standard deviation. This variation gives an additional uncertainty to the Standard Model 
$1.11 \Delta \chi^{2}=\chi^{2}-\chi_{\min }^{2}$ vs Higgs boson mass $M_{H}$. The line is the result of the fit. The band represents an estimate of the theoretical error due to missing higher order corrections. The vertical band shows the $95 \%$ C.L. exclusion limit on $M_{H}$ from the direct search. The dashed curve is the result obtained using the evaluation of

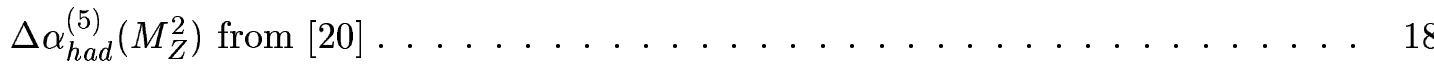

2.1 Elevation view of one half of the CDF II detector $\ldots \ldots \ldots$

2.2 Cross section of the CDF II plug calorimeter $\ldots \ldots \ldots . \ldots \ldots$

$2.3 \eta$ and $\phi$ coverage of the Run II muon systems $\ldots \ldots \ldots \ldots$

2.4 Block diagram of the CDF II Trigger $\ldots \ldots \ldots \ldots \ldots \ldots \ldots$

2.5 Design of the CDF online consumer framework. . . . . . . . . . . . . . 41

3.1 Generic correction scale factors for level 4 to 7 as a function of $p_{T}$ (left) and $\eta$ (right) of jets. . . . . . . . . . . . . . . 46

3.2 Dijet balance. The triangle is for data and open circle is for Monte Carlo simulation. There are three different regions of calorimeter called "Central" $(|\eta|<0.7)$, "Crack" or "Wall" $(0.7<|\eta|<1.3)$, and "Plug" $(|\eta|>1.3) \ldots \ldots \ldots \ldots$

3.3 Vertex display of double tags in run 167551, event 7969376. I.P. stands for the interaction point $($ primary vertex $) \ldots \ldots \ldots \ldots \ldots \ldots$

4.1 Vertex display of double tags in run 155145 , event $132579 \ldots \ldots$. . . . . . . 54

4.2 Lego plot of run 155145 , event 132579 , with an electron plus 3 jets $\ldots \ldots$. . . . 55

5.1 Met vs lepton isolation plot. Region D corresponds to our signal samples. . . . . 62

5.2 Background and $t \bar{t}$ signal expectation (based on measured $t \bar{t}$ cross section) as a function of jet multiplicity. Events with three or more jets are required to have $H_{T}>200 \mathrm{GeV} \ldots \ldots \ldots \ldots \ldots \ldots \ldots \ldots \ldots$

$5.3 H_{T}$ distribution of the 57 tagged events with three or more jets, compared to the expected background and $t \bar{t}$ signal (normalized to the theoretical cross-section of $6.7 \mathrm{pb})$

6.1 The response variables(transfer function) for $b$ jets(left) and $w$ jets(right) with

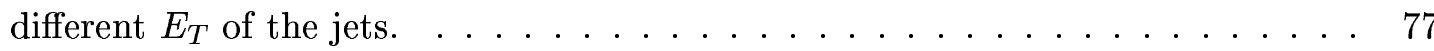


6.2 The response function in each detector $\eta$ of the jets bins for $b$ jets(left) and $w$ jets(right). Central $(<0.7)$ in black, Wall $(0.7<\eta<1.32)$ in red and $\operatorname{Plug}(>1.32)$ in blue. . . . . . . . . . . . . . . . . . . . . 78

6.3 Comparisons of reconstructed 2 jets $\mathrm{W}$ (left) and 3 jets top(right) invariant masses before and after transfer function applied. Masses right after generic corrections are shown in black and red line corresponds to the ones after generic corrections plus transfer function. . . . . . . . . . . . . . . 7

6.4 Comparisons of generic jet correction (black) and transfer function (red) in terms of reconstructed 2 jets $\mathrm{W}$ and 3 jets Top invariant masses: Detector $\eta$ of $\mathrm{W} /$ Top dependence(left) and $p_{T}$ of $\mathrm{W} /$ Top dependence(right). . . . . . . . . . 80

6.5 Pull distribution from 2000 sets of 22 events pseudo experiment of $t \bar{t}$ sample. Gaussian fit returned the mean of -0.05 and the sigma of $1.01 \ldots \ldots$. . . . . 82

6.6 The reconstructed top quark mass with different mass samples. The linear fit in red is from applying the transfer function of $M_{t o p}=175 \mathrm{GeV} / c^{2}$ to all mass samples. Blue line shows the results using the transfer function obtained from each mass samples. The slopes are 0.84 and 0.98 for red and blue fit respectively.

6.7 Sanity check. Reconstructed top quark mass as a function of its input top quark mass after taking into account mass shift by mapping functions. . . . . . . . . .

6.8 Width of Pull distribution as a function of input top quark mass after taking into account mass shift, which is consistent with a unit. . . . . . . . . . 85

6.9 The reconstructed mass (input $M_{t o p}=175 \mathrm{GeV} / c^{2}$ ) with varying the background fraction between $0 \%$ to $40 \%$. Each entry contains 22 events for pseudo experiments. 86

6.10 Mass shift $\left(M_{r e c}-M_{\text {input }}\right)$ due to individual background source using signal sample of $M_{t o p}=175 \mathrm{GeV} / c^{2}$ as a function of background fraction. Solid red line represents all combined background with expected number of events in Table 5.10. 87

6.11 The reconstructed mass as a function of input mass with the background fraction from $0 \%$ to $40 \%$. . . . . . . . . . . . . . . . 88

6.12 The slope of linear fit in figure 6.11 as a function of the background fraction. This can be used as a mapping function to extract the top quark mass from background pulling. . . . . . . . . . . . . . . . . . .

6.13 Sanity check after applying the mapping functions with a background of $19 \%$. The slope of the linear fit between the reconstructed top quark mass and its input mass is consistent with $1.0 \ldots \ldots \ldots \ldots \ldots$ 
6.14 The width of the mass pull distribution, which is consistent with 1.0 . . . . . 90

6.15 The results of blind tests. Plots are $\Delta M=M_{r e c}-M_{\text {true }}$ from low to high top quark mass. . . . . . . . . . . . . . . . . . . . . 91

7.1 The joint negative $\log$ likelihood distribution of the 22 events gives $M_{t o p}=$ $176.5_{-3.8}^{+3.4} \mathrm{GeV} / \mathrm{c}^{2} \ldots \ldots \ldots \ldots \ldots \ldots \ldots \ldots \ldots$

7.2 Extracted top quark mass using the mapping function as a function of the background fraction. . . . . . . . . . . . . . . . 95

7.3 Estimated statistical uncertainties on top quark mass as a function of the background fraction. . . . . . . . . . . . . . . . 96

7.4 The expected positive and negative statistical uncertainties from 22-event pseudoexperiments from Monte Carlo events. An input top quark mass of $175 \mathrm{GeV} / \mathrm{c}^{2}$ is used for the comparison. The black arrows indicate those in the data. . . . . .

8.1 The systematic shift of the reconstructed mass from 1000 pseudo experiments due to jet energy correction (level 1: Relative correction) uncertainty. $+\sigma$ in blue, $-\sigma$ in red for the correction, where input top quark mass is $175 \mathrm{GeV} / c^{2} \ldots \ldots . . .102$

9.1 Event likelihood distribution. The number of signal and background events is normalized to the number of observed events (22 events). Blue is signal events and green is background, The red line corresponds to the combined Monte Carlo expectation. The triangles are the 22 data events. . . . . . . . . . . . 108

9.2 Maximum mass distribution in data and Monte Carlo. The signal Monte Carlo sample is for $\mathrm{M}_{t o p}=175$. The first(last) bin includes under(over)flow.Maximum mass distribution in data and Monte Carlo. The signal Monte Carlo sample is for $\mathrm{M}_{\text {top }}=175$. The first(last) bin includes under(over)flow . . . . . . . . . . 109

10.1 Summary of the best top quark mass measurements obtained from RunII Tevatron experiments so far. The result from DLM is referred as CDF Lepton+jets which is the best of all, and consistent with the world average extracted by an combination of all Run I results (blue bottom). . . . . . . . . . . . . . . . . . 112 


\section{List of Tables}

1.1 Fundamental Particles of the Standard Model. . . . . . . . . . . . . . . 4

1.2 Branching Ratios for $t \bar{t}$ decay modes in standard model coupling. $q$ stands for a $u, d, c$ or $s$ quark. Events are categorized into four channels: All-jets, Lepton+jets, Dilepton and $\tau$ channels. . . . . . . . . . . . . . . . 10

2.1 Evolution of Tevatron parameters . . . . . . . . . . . . . . 20

2.2 Evolution of Tevatron parameters . . . . . . . . . . . . . 21

2.3 Calorimeter segmentation . . . . . . . . . . . . . . 29

2.4 Characteristics of the CDF II calorimeter . . . . . . . . . . . 30

2.5 Parameters of muon detection at CDF . . . . . . . . . . . 35

4.1 The numbers of observed events with at least one $b$ tagged jet. . . . . . . 53

5.1 Summary table of the $t \bar{t}$ acceptance, for a top quark mass of $175 \mathrm{GeV} / c^{2}$. . . 60

5.2 Relative systematic uncertainties on the signal acceptance which are common to all lepton types. . . . . . . . . . . . . . . . . . 61

5.3 non- $W$ QCD background estimate. Results from the tag rate method and the tag sample method are the number of events expected in the b-tagged lepton + jets sample. . . . . . . . . . . . . . . . .

5.4 Background summary for the single-tag selection. The total backgrounds are given before and after the correction for $t \bar{t}$ events in the pretag $W+$ jets sample. . 65

5.5 Measured cross section for different top quark mass assumptions. . . . . . . 66

5.6 The numbers of expected events for individual background sources in $\mathrm{W}+4 \mathrm{jets}$ sample. ................................ 67

6.1 The comparison of the 2jets/3jets mass of $\mathrm{W} /$ top before and after the transfer function applied. $<$ Mean $>$ and RMS are shown. . . . . . . . . . 79 
6.2 The maximum likelihood masses for various background sources. . . . . . . . 85

8.1 Systematic uncertainties on the relative jet corrections (Level 1) for data and Monte Carlo simulation. . . . . . . . . . . . . . . . . . . 101

8.2 The systematic uncertainties for jet energy corrections in individual levels. Two entries in Level 1 and 3 are due to the correction factor differences between Monte Carlo simulation $(\mathrm{MC})$ and Data. . . . . . . . . . . . . . 101

8.3 The summary of systematic uncertainties . . . . . . . . . . . 105 


\section{Chapter 1}

\section{Introduction}

The aim of high-energy physics is to expand the limits of human knowledge of the nature/universe by identifying the constituents of matter at the most fundamental level and describing the interactions between them. To do this, high-energy physicists have desired to probe the structure of particle at smaller scales of distance, that is higher scales of energy. For more than a decade, the studies at the frontier of particle energy have been established at the Fermi National Accelerator Laboratory (Fermilab) in Batavia, IL in the U.S.A., colliding beams of protons $(p)$ and antiprotons $(\bar{p})$ counter-rotating in a 6.4-kilometer ring with super-conducting magnets called the Tevatron. The accelerator is designed such that these collisions occur at two points, where the out-coming particles from collisions are observed using immense detectors. The analysis presented in this dissertation was completed by using data from the Collider Detector at Fermilab (CDF) experiment, one of these collision points.

One of the important roles of experiments at the energy frontier is to search for particles which are too massive to have been produced at any previous experiments. In fact, in 1995 at the Fermilab Tevatron accelerator, known as Run I (1992-1996), which provided $p \bar{p}$ collisions at a center-of-mass energy of $1.8 \mathrm{TeV}$, the discovery of the top quark marked the end of a long and difficult search. This was also another success for the Standard Model of particle physics (SM), which had strongly predicted its existence as an isospin doublet partner of the bottom quark discovered in 1977. The top quark is by far the heaviest fundamental particle known, and this large mass close to the electroweak symmetry breaking (EWSB) scale has caused much excitement in the particle physics.

For Run II at the Fermilab carried out since 2001 with upgraded detectors (CDF II) and an 
increase in the center-of-mass energy of the Tevatron from 1.8 to $1.96 \mathrm{TeV}$, the strategy of top quark analysis is to move from the discovery mode of Run I and begin to develop the paradigm for higher statistics measurements of the top sector.

The goal of this dissertation is to measure one of the top quark properties, the mass, with the highest possible accuracy by using the best knowledge of its production and decay mechanism described by the SM. The integrated luminosity of the data sample used for this analysis, collected from March 2002 through August 2003 during Run II, is $162 \mathrm{pb}^{-1}$ which is 1.5 times more than the data obtained during Run I. In addition to measurement of the top quark mass, $t \bar{t}$ production cross section was also measured by using same datasets and same channels.

In this chapter, we summarize a short review of the theoretical understanding of the Standard Model and focus on describing $t \bar{t}$ production and decay in addition to the role of top quark in high-energy physics. Chapter 2 describes the accelerators at Fermilab called Tevatron, which are currently the world's only source of top quarks, and the CDF II detector, which allows us to study them. Chapter 3 discusses the identification of leptons, missing transverse energy, jets and their corrections. Chapter 4 presents the details of data samples used for this analysis and event selection to obtain $t \bar{t}$ candidates. Chapter 5 details $t \bar{t}$ production cross section measurements which highly related to our estimation of backgrounds. Chapter 6 describes the method of this analysis used in this dissertation, DLM. Chapter 7 summarizes the results obtained using Run II data. Chapter 8 contains studies of systematic uncertainty, and Chapter 9 presents additional checks of the method by comparing Monte Carlo samples and data. Lastly, Chapter 10 concludes the dissertation with a short discussion.

\subsection{The Standard Model}

The Standard Model is a quantum filed theory based on the gauge symmetry groups $\mathrm{SU}(3)_{C}$ $\times \mathrm{SU}(2)_{L} \times \mathrm{U}(1)_{Y}$. The first gauge group, $\mathrm{SU}(3)_{C}$, corresponds to the strong force of Quantum Chromodynamics (QCD) [1], and the second and third gauge groups, $\mathrm{SU}(2)_{L} \times \mathrm{U}(1)_{Y}$, correspond to the symmetry of the electroweak interactions in which $\mathrm{SU}(2)_{L}$ corresponds to the left handed $\mathrm{SU}(2)$ symmetry and $\mathrm{U}(1)_{Y}$ the hypercharge symmetry, respectively, and eventually these gauge interactions are broken into the V-A theory of weak interaction [2] and the Quantum Electrodynamics (QED) [3]. There is another force in nature, gravitation. It is however no likely to influence subatomic processes since level of the force is so much weaker than the other three interactions. Moreover, unlike the other interactions, gravitation is not yet incorporated 
into the standard model.

In the Standard Model there are three types of fundamental particles: the leptons, the quarks and the gauge bosons. The leptons and the quarks are spin- $1 / 2$ particles called fermions which obey the Pauli exclusion principle. They are the constituents of matter and arranged in three families of weak isospin doublets. In addition, every particle has its own anti-particle which is identical in terms of mass and spin, but has opposite electric charge. Anti-particles are denoted by placing bars above the particle symbols.

The known leptons are the electron $\left(e^{-}\right)$, the muon $\left(\mu^{-}\right)$and the tau $\left(\tau^{-}\right)$with electric charge $\mathrm{Q}=-1$, and the corresponding neutrinos $\nu_{e}, \nu_{\mu}$ and $\nu_{\tau}$ with electric charge $\mathrm{Q}=0$. The known quarks are categorized into 6 types, or flavor, called up $(u)$, down $(d)$, charm $(c)$, strange $(s)$, top $(t)$ and bottom $(b)$ with fractional electric charge of an electron $(1 / 3$ or $2 / 3)$. Unlike the leptons, quarks experience strong interactions in addition to electromagnetic and weak interactions. Besides electric charge, each quark also carries a "color" charge of either red, green or blue. This color charge of the strong force is well described in QCD and is analogous to the electric charge of the electromagnetic force. The gauge bosons are the mediators of the fundamental forces and obey Bose-Einstein statistics due to their integral spin. The interaction between any two particles can be visualized as a process in which two particles exchange a virtual gauge boson. The known gauge bosons are photon $(\gamma), W^{ \pm}, Z^{0}$ and gluon $(g)$. The electromagnetic interaction is mediated by the photon and involves all particles that have electric charge. The photon is chargeless, massless and it does not interact with itself. Its coupling strength increases logarithmically with the energy of the interaction [4]. The strong force is mediated by eight different gluons. The gluons are massless, electrically neutral and carry color charge which means that they not only interact with quarks but also with themselves. The strength of the strong coupling gets smaller as the energy of the interaction increases [5]. The weak interaction is mediated by the $W$ with electric charge $\mathrm{Q}= \pm 1$ and electrically neutral boson, $Z$. These particles are very massive, and the fact that their masses are close to 100 $\mathrm{GeV} / \mathrm{c}^{2}$, indicates that their interactions are characterized by small distances. Table 1.1 shows a summary of the properties of the fundamental particles. To enhance that the mass of top quark is very heavy compared to other quarks, Figure 1.1 describes a comparison of masses of standard model quark. Clearly, the top quark has large mass which is more than 35 times larger than next heaviest quark, bottom.

The quarks behave as almost free particles at high energies and their interactions can be calculated using perturbative methods. When energies are low, these methods cannot be used 


\begin{tabular}{|c|cccccc|}
\hline \hline & First & Second & Third & Interaction & Charge (Q) & Spin \\
\hline Leptons & electron $\left(e^{-}\right)$ & muon $\left(\mu^{-}\right)$ & tau $\left(\tau^{-}\right)$ & EM, Weak & -1 & $1 / 2$ \\
& $e$-neutrino $\left(\nu_{e}\right)$ & $\mu$-neutrino $\left(\nu_{\mu}\right)$ & $\tau$-neutrino $\left(\nu_{\tau}\right)$ & Weak & 0 & $1 / 2$ \\
\hline \multirow{2}{*}{ Quarks } & up $(u)$ & charm $(c)$ & top $(t)$ & EM, Weak, Strong & $+2 / 3$ & $1 / 2$ \\
& down $(d)$ & strange $(s)$ & bottom $(b)$ & EM, Weak, Strong & $-1 / 3$ & $1 / 2$ \\
\hline \hline \multirow{2}{*}{ Gauge } & Name & Force & Coupling & Mass $\left(\mathrm{GeV} / \mathrm{c}^{2}\right)$ & Charge $(\mathrm{Q})$ & Spin \\
\cline { 2 - 5 } Bosons & Photon $(\gamma)$ & EM & $10^{-2}$ & 0 & 0 & 1 \\
& boson $(W)$ & Weak & $10^{-13}$ & 80.4 & \pm 1 & 1 \\
& Z boson $(Z)$ & Weak & $10^{-13}$ & 91.2 & 0 & 1 \\
& Gluon $(g)$ & Strong & 1 & 0 & 0 & 1 \\
\hline \hline
\end{tabular}

Table 1.1: Fundamental Particles of the Standard Model.

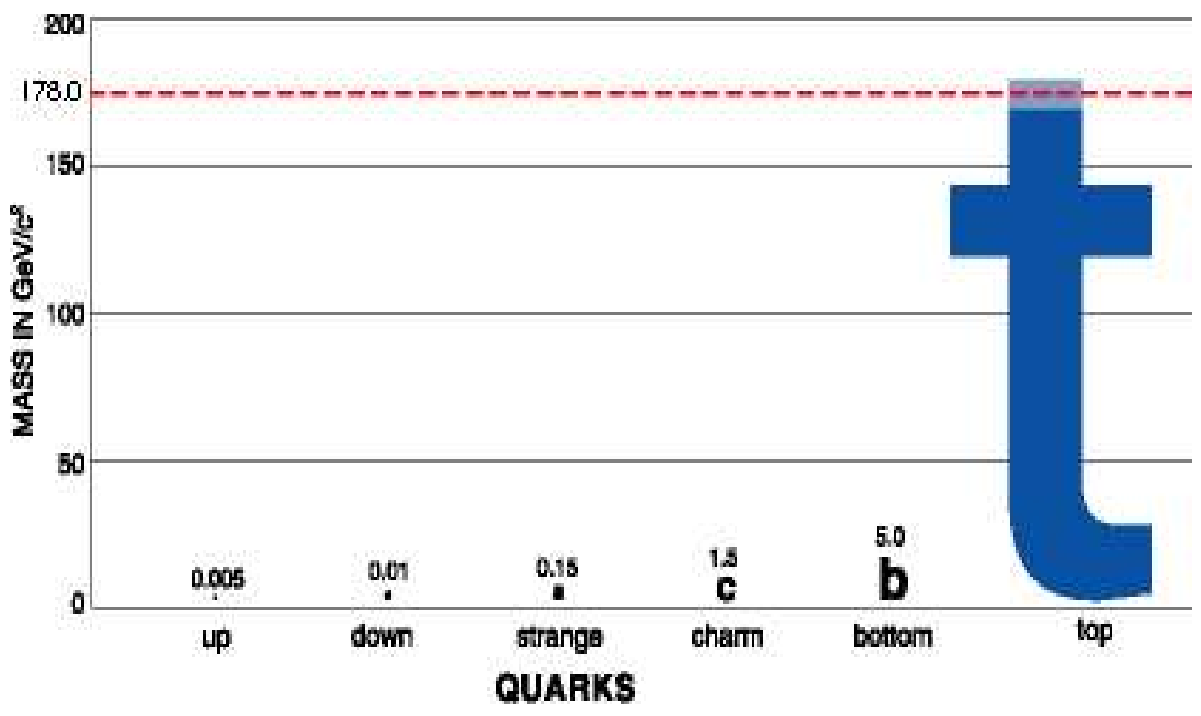

Figure 1.1: Comparison of masses of Standard Model quarks [6]. 
because the coupling becomes strong. A technique called resummation has been developed to minimize the effects of singularities, to avoid divergent terms by re-ordering of the perturbative series [5]. As a consequence, the quarks are bound together through the strong force to form two types of hadrons: mesons and baryons. Mesons are bosons (color-anticolor). For example, the $\pi^{+}$particle is composed of a $u$ and a $\bar{d}$ quarks. Baryons are fermions which consist of three quarks or anti-quarks. For example, the proton is comprised of $2 u$ quarks and $1 d$ quark. Namely, the quarks form combinations in which the sum of their electric charges is an integer and the sum of their color charges is neutral(color-anticolor or (anti)red $+($ anti)blue + (anti)green). Therefore, when quarks or gluons are produced in collisions, they are not observed as such, but transformed to hadrons in a complex evolution from objects with color to colorless hadrons. This kind of process is called fragmentation. We detect hadrons that move in the same direction as the original quark or gluon. This fragmented parton (quark or gluon) is referred to as a jet from experimental point of view.

In the standard model, particles acquire their mass via the "Higgs mechanism". In order to accommodate electroweak and flavor symmetry breaking, we must artificially introduce a weak-isospin doublet of fundamental scalar field $\Phi=\left(\phi^{-}, \phi^{0}\right)$ with a potential of the form,

$$
V\left(\Phi^{\dagger} \Phi\right)=\mu^{2}\left(\Phi^{\dagger} \Phi\right)+|\lambda|\left(\Phi^{\dagger} \Phi\right)^{2}
$$

where $\lambda$ is the self coupling of the scalar field. If $\mu^{2}$ is chosen to be negative, the electroweak symmetry is spontaneously broken when the field is expanded about its non-zero vacuum expectation value $v=\sqrt{-\mu^{2} / \lambda}=\left(G_{F} \sqrt{2}\right)^{-1 / 2}=246 \mathrm{GeV}$. This value is referred to as the electroweak scale. The spontaneous symmetry breaking gives rise to the $\mathrm{W}$ and $\mathrm{Z}$ bosons, and it also gives rise to a spin-0 (scalar) particle called the Higgs boson. Each quark and lepton has its own Yukawa-coupling to the Higgs boson $G_{f}$ and thus acquire a mass $m_{f}=G_{f} v / \sqrt{2}$, where small $\mathrm{f}$ stands for fermion. Since the strength of this coupling to each fermion is proportional to their own mass, the top quark, due to its surprisingly large mass, plays a special role for the Higgs boson.

The Higgs boson has not been yet discovered, as of the time of this dissertation.

\subsection{Top Quark Physics}

The top quark is the heaviest elementary particle yet discovered. Its mass, of the same order as the electroweak scale $(v / \sqrt{2})$, is about twice that of the $\mathrm{W}$ and $\mathrm{Z}$ bosons and about 35 times 
larger than its isospin partner, the b quark. Because of its large mass, it can only be produced in the collisions of the particles where a sufficiently high center-of-mass energy $(\sqrt{s})$ can be achieved. The $p \bar{p}$ accelerator, Tevatron, at Fermilab $(\sqrt{s}=1.96 \mathrm{TeV})$ has been the only facility in the world where the top quarks have been produced.

\subsubsection{Discovery}

Fifth quark, the bottom quark, was discovered in 1977 at the Fermilab by a fixed target experiment where energetic protons were directed onto a stationary atom [7]. There had not been evidence of the existence of the top quark in accelerators up to that point. To discover the top quark, situations where there would be more energy available to create heavier particles was needed. This condition was finally achieved by hadron collider, where protons and anti-protons traveling in opposite directions are made to collider head on. The top quark was first observed in 1995 at the Fermilab Tevatron collider by the CDF and D $\varnothing$ collaborations [8]. There were only about 100 total top candidate events found during this entire period of data taking which spanned 5 years known as Run I (1992-1996).

\subsubsection{Production and Decay}

\section{$t \bar{t}$ pair production}

In hadron collider at center-of-mass energy of $1.96 \mathrm{TeV}$, top quarks are expected to be produced mainly as a pair by two distinct processes in which both are via gluon described by QCD strong interaction: $q \bar{q}$ annihilation and gluon-gluon $(g g)$ fusion. Figure 1.2 shows these Feynman diagrams at the leading order. At the Tevatron, the relative contributions from these two processes are about $85 \%$ and $15 \%$ respectively, including Next-to-Leading Order (NLO) contribution.

The total cross section for the production of heavy quarks is given by,

$$
\sigma(p \bar{p} \rightarrow t \bar{t})=\sum_{i, j} \int d z_{i} d z_{j} f_{i / p}\left(z_{i}, \mu^{2}\right) f_{j / \bar{p}}\left(z_{j}, \mu^{2}\right) \hat{\sigma}\left(i j \rightarrow t \bar{t} ; \hat{s}, \alpha_{s}\left(\mu^{2}\right), M_{t o p}\right),
$$

where the sum is over all partons: gluons, light quarks and antiquarks. This formula expresses the total cross section in terms of the parton-parton processes $i j \rightarrow t \bar{t}$. The parton distribution function $f_{i / p}$ corresponds to the probability density of finding a parton with a give fraction of the proton momentum between $z_{i}$ and $z_{i}+d z_{i}$. The function $f_{j / \bar{p}}$ has the same definition but for the antiproton. The short-distance cross section for the parton-parton subprocess is $\hat{\sigma}$. The 

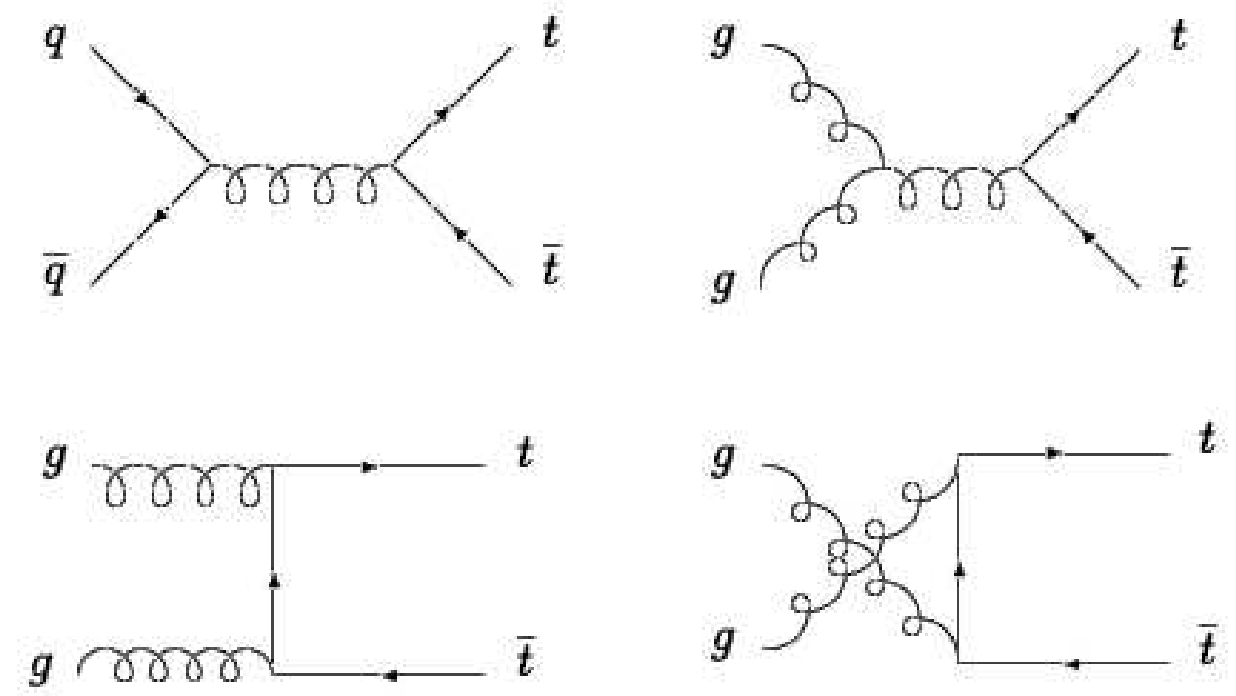

Figure 1.2: The lowest order diagrams for $t \bar{t}$ production at the Tevatron

center-of-mass energy of the $i-j$ parton system is given by $\hat{s}$ and it is related to the $p \bar{p}$ center-ofmass energy by $\hat{s}=z_{i} z_{j} s$. The parameter $\mu$ is the renormalization scale which is introduced to include the resultant contribution from higher order Feynman graphs. If the calculation could be carried out to all orders then the dependence on $\mu$ would vanish. The strong coupling constant is give by $\alpha_{s}$ and $M_{t o p}$ is the mass of top quark. The current theoretical calculation of the $t \bar{t}$ production cross section at the center-of-mass energy of $1.96 \mathrm{TeV}$, with a top quark mass of 175 $\mathrm{GeV} / c^{2}$, is approximately $6.7 \pm 0.9 \mathrm{pb}[9]$. Figure 1.3 shows a comparison between Run I and Run II for the experimental results and the standard model calculation by theory.

\section{Top quark standard model decay}

In the standard model, a top quark decays almost $100 \%$ of the time into a $W$ boson and a $b$ quark. There are other decays possible but these are strongly suppressed since they involve off-diagonal elements of the Cabbibo-Kobayashi-Masukawa (CKM) matrix which are close to zero. Although there are additional theories that can change the standard model predictions, this analysis considers only the top decay channel $t \rightarrow W b$. The $W$ boson from the top quark decays into either a lepton and its neutrino or a $q \bar{q}^{\prime}$ pair. Figure 1.4 shows the Feynman diagram for top production by $q \bar{q}$ annihilation and the subsequent top quark decay.

The decay modes of the $W$ boson determine an event topology and are used as a way to 


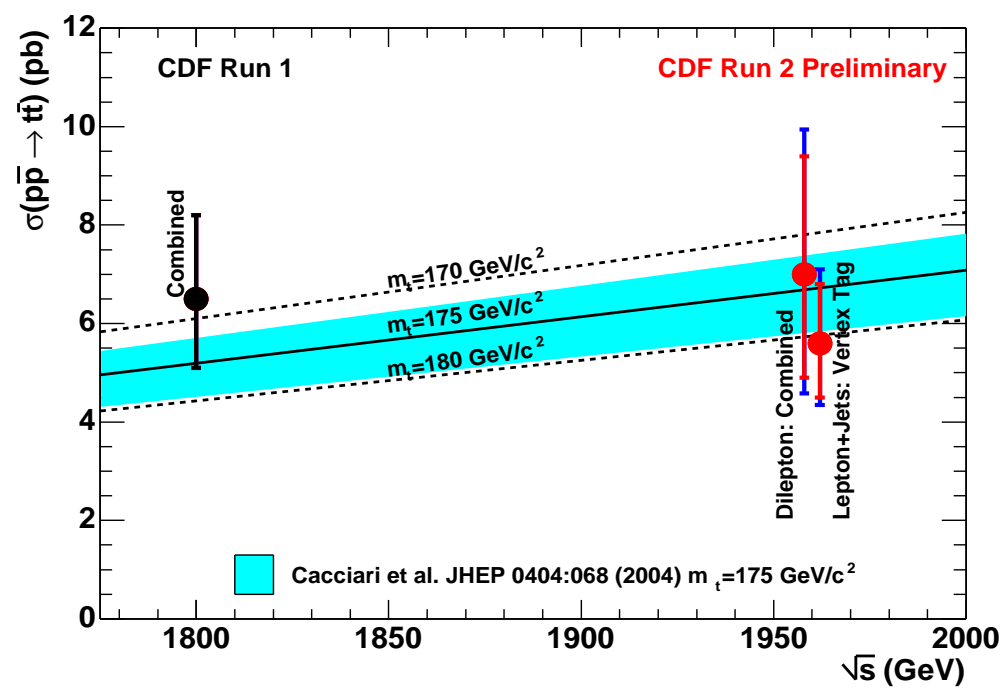

Figure 1.3: Comparison of $t \bar{t}$ production cross section between Run I $(\sqrt{s}=1.8 \mathrm{TeV})$ and Run II $(\sqrt{s}=1.96 \mathrm{TeV})$ for the experimental results (points) and the Standard Model calculation by theory (blue band).

classify the events. Table 1.2 lists the final states of $t \bar{t}$ production, according to the $W$ decay modes. The events are classified as either an "all-hadronic", "lepton+jets", "dilepton" or " $\tau$ channel". An event is considered as an all-hadronic event when both $W$ boson decay to a quarkanitquark pair, $t \bar{t} \rightarrow\left(q \bar{q}^{\prime} b\right)\left(q \bar{q}^{\prime} \bar{b}\right)$. The signature in this mode is six or more jets. Though this channel has the largest branching fraction, 44\%, it has a huge amount of background from other QCD multijets production process. The analysis using all-hadronic channel has been described extensively elsewhere [8]. An event is classified as a dilepton event when both $W$ bosons decay leptonically to an $e$ or $\mu$. This channel is identified by two high- $p_{T}$ leptons and large $\mathbb{F}_{T}$ from the leptonic decay of both $W \mathrm{~s}$, and two jets from the hadronization of the $b$ quarks. The dilepton channel has the least background contamination but a very small branching fraction, $5 \%$. The backgrounds in this mode come from direct $b \bar{b}, W W, Z \rightarrow \tau \tau$, Drell-Yan production and lepton misidentification. The analysis using dilepton channel has been described elsewhere [8]. An event which contains $\tau$ lepton in the final state is classified as $\tau$ channel. $\tau$ lepton is considered as more closer to jets rather than other leptons ( $e$ or $\mu$ ), since $\tau$ decays predominantly into charged and neutral hadrons. Hadronic decays happen with a total branching fraction of $64 \%$ (leptonic 
decay with 36\%: $\left.\tau \rightarrow e(\mu) \nu \nu_{\tau}\right)$. There is always at least one $\nu$ in the final state. Therefore, the identification of $\tau$ lepton is more complicated than $e$ and $\mu$ leptons. The CDF has however, successfully identified and obtained physics results using $\tau$ leptons described elsewhere [10].

This analysis focuses on events in which one $W$ decays to a lepton-neutrino pair and the other $W$ decays to hadronically. These events are classified as lepton+jets events. The signature for this channel is a charged high- $p_{T}$ isolated lepton $(e$ or $\mu)$, an imbalance in energy from the undetected neutrino called missing transverse energy $\left(\mathscr{F}_{T}\right)$, and four (or more) jets from the hadronized quarks. Requiring one of the $W$ bosons to decay leptonically to an $e$ or $\mu$ substantially reduces the amount of background without significantly reducing the branching fraction, $30 \%$. Backgrounds to this channel come predominantly from higher order production of $W$ bosons, where the $W$ recoils against significant jet activity. This process is referred to as " $W+$ multijet" background. Figure 1.5 shows one of the Feynman diagrams for QCD $W+$ multijet production. Since every top quark has two jets from two $b$ quarks, the $W+$ multijet background can be greatly reduced by identifying (or tagging) at least one of the $b$ quarks in the event. The method we use for $b$-tagging utilizes the $b$ 's lifetime. This long lifetime which is $\sim 1.5$ ps means that the $b$ quarks will form a $B$ hadron and travel on average a few millimeter before decaying. $B$ hadrons can be detected experimentally by finding jets with vertices displaced from the primary vertex of the event. Section 3.3 explains this algorithm in more details.

After requiring at least one $b$ tagged jet in the event, main backgrounds are $W$ theavy flavor $(b \bar{b}, c \bar{c})$, QCD with fake leptons so called non-W events, and mistagging of $b$ jets due to finite tracking resolutions. Complete estimations of these background in our sample are described in section 5.3.

\subsection{3 $t \bar{t}$ Cross Section Measurements}

The first task in an experimental top physics is to verify that they are made as expected by measuring the $t \bar{t}$ production cross section $\left(\sigma_{t \bar{t}}\right)$. This measurement is sensitive to new physics. Because the standard model (QCD) predicts the production rate expected from $t \bar{t}$ pairs, given a mass of the top quark and the center-of-mass energy of the colliding beams. Any discrepancy between experimental data and the prediction could indicate non-standard production mechanisms or couplings of the top quark.

In general, the cross section is measured by,

$$
\sigma_{t \bar{t}}=\frac{N_{o b s}-N_{b k g}}{\epsilon_{t \bar{t}} \cdot \int L d t} \quad \text { or } \quad \frac{N_{o b s} \times f_{s i g}}{\epsilon_{t \bar{t}} \cdot \int L d t}
$$




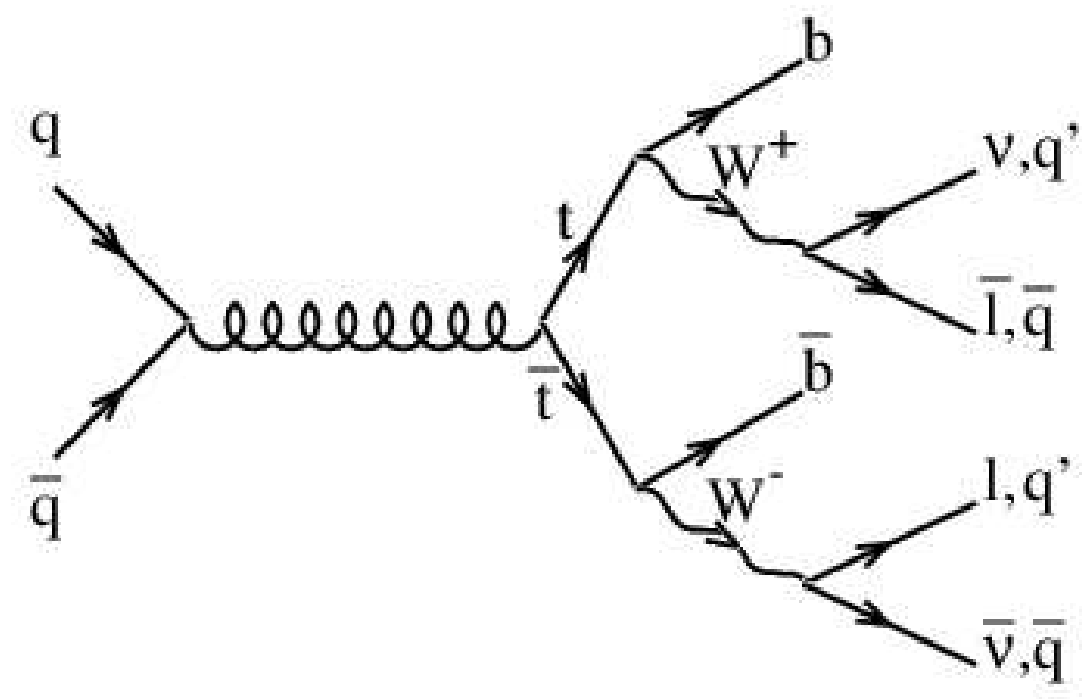

Figure 1.4: The tree-level Feynman diagram for $t \bar{t}$ production by $q \bar{q}$ annihilation and its standard model decay chain.

\begin{tabular}{lcc}
\hline \hline Decay mode & Branching Ratio & Channel Category \\
\hline$t \bar{t} \rightarrow\left(q \bar{q}^{\prime} b\right)\left(q \bar{q}^{\prime} \bar{b}\right)$ & $36 / 81$ & All-hadronic \\
$t \bar{t} \rightarrow\left(q \bar{q}^{\prime} b\right)(e \nu \bar{b})$ & $12 / 81$ & Lepton+jets \\
$t \bar{t} \rightarrow\left(q \bar{q}^{\prime} b\right)(\mu \nu \bar{b})$ & $12 / 81$ & Lepton+jets \\
$t \bar{t} \rightarrow\left(q \bar{q}^{\prime} b\right)(\tau \nu \bar{b})$ & $12 / 81$ & $\tau$ channel \\
$t \bar{t} \rightarrow(e \nu b)(\mu \nu \bar{b})$ & $2 / 81$ & Dilepton \\
$t \bar{t} \rightarrow(e \nu b)(\tau \nu \bar{b})$ & $2 / 81$ & $\tau$ channel \\
$t \bar{t} \rightarrow(\mu \nu b)(\tau \nu \bar{b})$ & $2 / 81$ & $\tau$ channel \\
$t \bar{t} \rightarrow(e \nu b)(e \nu \bar{b})$ & $1 / 81$ & Dilepton \\
$t \bar{t} \rightarrow(\mu \nu b)(\mu \nu \bar{b})$ & $1 / 81$ & Dilepton \\
$t \bar{t} \rightarrow(\tau \nu b)(\tau \nu \bar{b})$ & $1 / 81$ & $\tau$ channel \\
\hline \hline
\end{tabular}

Table 1.2: Branching Ratios for $t \bar{t}$ decay modes in standard model coupling. $q$ stands for a $u, d, c$ or $s$ quark. Events are categorized into four channels: All-jets, Lepton+jets, Dilepton and $\tau$ channels. 


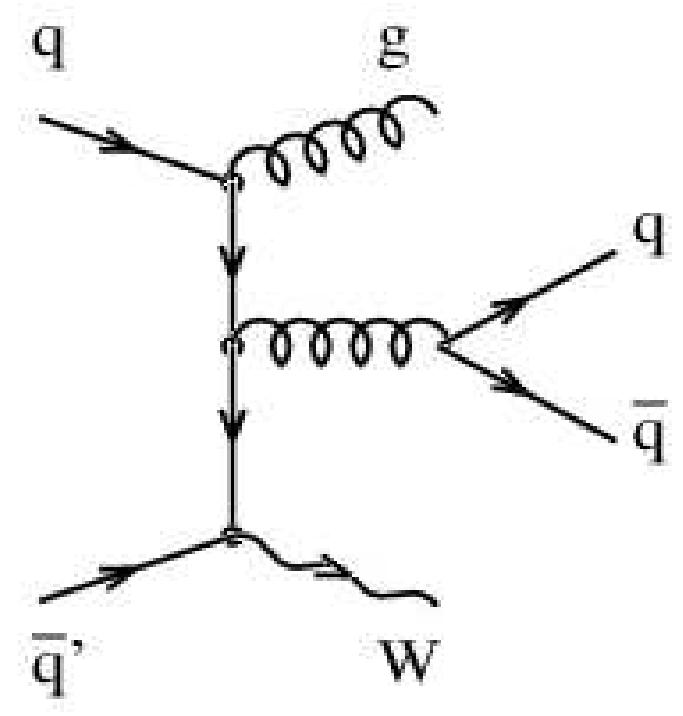

Figure 1.5: The Feynman diagram for $W+$ multijet production which is one of the backgrounds to the top signal.

Namely, it is obtained by the number of observed events candidates minus the prediction for observed background events, divided by the product of the event selection efficiency and the integrated luminosity of the data sample. When the signal fraction in the candidates is obtained instead of the predicted number of backgrounds events, the numerator can be replaced with the number of observed events candidates multiplied by the fraction of signal $t \bar{t}$ events $\left(f_{\text {sig }}\right)$. CDF has measured it by using different techniques with different final states. All results obtained by CDF in Run II so far are summarized in Figure 1.6. All of them are consistent with each other and the prediction from theory within their uncertainties. In order to measure the top quark mass, background estimation is also necessary to take into account their contribution to the mass measurement. Chapter 5 describes more details of the measurement of the cross section using (1) Counting Method, (2) Kinematic Fitting Method with lepton+jets btagged events which is the same topology used for this analysis.

\subsection{The Mass of Top Quark}

The top quark mass, $M_{t o p}$ is an important ingredient in particle physics. Its precise measurement not only serves for setting basic parameters in calculations of electroweak processes but also 


\section{Top Pair Production Cross Section}

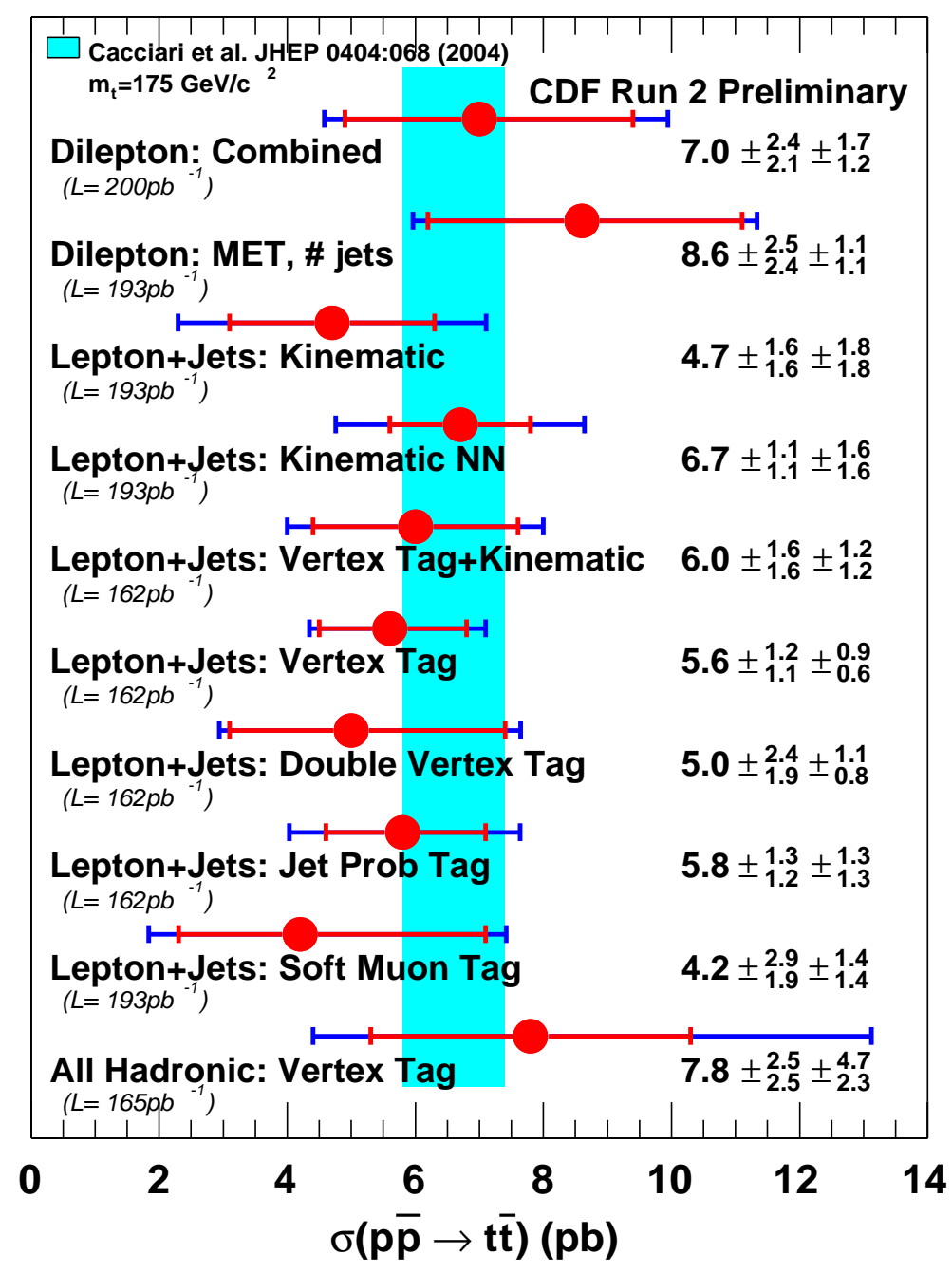

Figure 1.6: Comparison of measured values of $t \bar{t}$ production cross section $\left(\sigma_{t \bar{t}}\right)$ at CDF Run II. 
provides a constraint on the mass of Higgs boson. Therefore it is desirable to have a measurement with a precision comparable to that of other electroweak parameters, typically of the order of $1 \%$, corresponding about $2 \mathrm{GeV} / c^{2}$ in $M_{t o p}$. This section describes how well $M_{t o p}$ has been measured so far, and also its relations to the Higgs boson.

\subsubsection{Results from the Tevatron in Run I and Run II}

Based on Run I Tevatron data at the center-of-mass energy of $1.8 \mathrm{TeV}$, the CDF and DØ collaborations have published several direct experimental measurements of $M_{t o p}$ with all decay topologies arising $t \bar{t}$ production: the dilepton channel $[11,12]$, the lepton+jets channel $[13,14]$, and the all-jets channel analyzed by CDF only [15]. Figure 1.7 shows a summary of measured $M_{\text {top }}$ values. By adding a recent reanalysis of $\mathrm{D} \emptyset \mathrm{RunI}$ data [16], the current world average of the top quark mass is $178.0 \pm 2.7 \pm 3.3 \mathrm{GeV} / c^{2}$ (Total uncertainty of $\pm 4.3 \mathrm{GeV} / c^{2}$ )[17]. The results in Run I are all summarized in Figure 1.7.

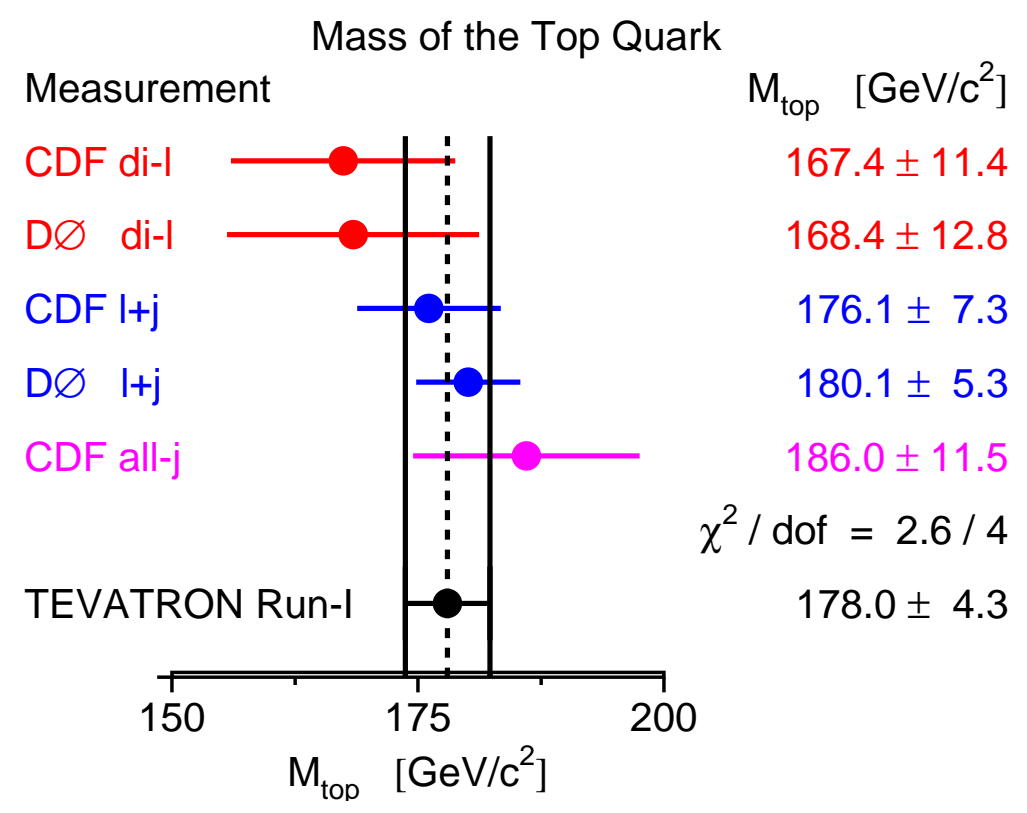

Figure 1.7: Summary of the top quark mass measured by using Run I Tevatron data. di-l, $1+\mathrm{j}$ and all-j stand for dilepton, lepton+jets and all-hadronic channels respectively.

On the other hand, based on Run II Tevatron data at the center-of-mass energy of $1.96 \mathrm{TeV}$, the top quark mass has been measured. Figure 1.8 shows that the results obtained by CDF so 
far. Since Run II experiment is ongoing as of the time of this paper, those are very preliminary and not final values of CDF. Using an integrated luminosity of $162 \mathrm{pb}^{-1}$ (with $b$ tagging) and $200 \mathrm{pb}^{-1}$ (without $b$ tagging requirement), CDF has obtained consistent results within different channels and techniques.

\subsubsection{Relation and Constraint on the Higgs Boson Mass}

As previously mentioned, the mass of the top quark enters into calculations of higher-order (radiative) corrections which connect electroweak processes depend on the masses of the top quark and the Higgs boson via loop diagrams such as those shown in Figure 1.9.

At one loop, for instance, the $\rho$ parameter,

$$
\rho=\frac{M_{W}^{2}}{M_{Z}^{2}}\left(1-\sin ^{2} \theta_{W}\right) \equiv 1+\Delta r
$$

which relates the $W$ and $Z$ boson masses and the weak angle, gets a radiative correction

$$
\Delta r=\frac{3 G_{F}}{8 \pi^{2} \sqrt{2}} M_{t o p}^{2}+\frac{\sqrt{2} G_{F}}{16 \pi^{2}} M_{W}^{2}\left[\frac{11}{3} \ln \left(\frac{M_{H}^{2}}{M_{W}^{2}}\right)+\cdots\right]+\cdots
$$

which is quadratic in the top mass. Note, however, that the dependence on the mass of the Higgs boson is only logarithmic. Therefore, the mass of the top quark is the dominant term in the corrections for electroweak process. By measuring $M_{t o p}$ very accurately, and using additional constraints from the large body of precision electroweak data we can test for consistency and predict unknowns in the standard model. One of such predictions is the mass of the Higgs boson, which can be constrained by the direct measurement of the W boson mass and $M_{t o p}$ along with other precision data. The indirect measurements of $M_{W}$ and $M_{t o p}$ based on data from the Large Electron Positron Collider (LEP) are shown in Figure 1.10, compared with the direct measurement (LEP-II with Tevatron Run I results). Also shown are the standard model predictions for Higgs boson masses between 114 and $1000 \mathrm{GeV}$. As can be seen in the figure, the indirect and direct measurements of $M_{W}$ and $M_{\text {top }}$ are now in good agreement, and both sets prefer a low value of the Higgs boson mass.

\section{Standard Model Global Fit for the Higgs Boson}

Particular interest associated with the top quark mass is the constraint on the mass of the Higgs boson, because this fundamental ingredient of the standard model has not been observed yet. Figure 1.11 shows the Delta-chi2 $\left(\Delta \chi^{2}\right)$ curve derived from high- $Q^{2}$ precision electroweak 


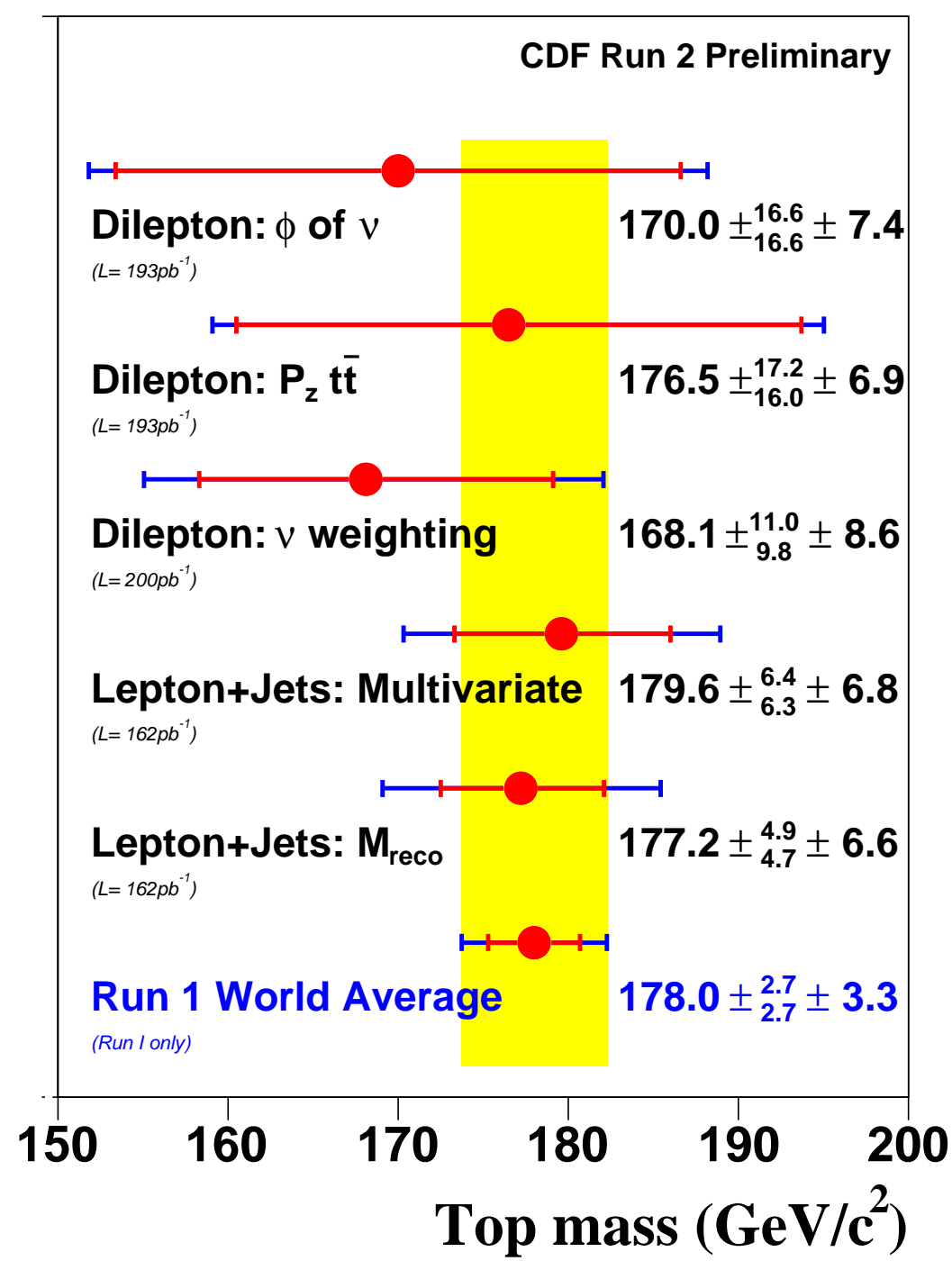

Figure 1.8: Summary of the top quark mass measured by using Run II Tevatron data obtained so far. Three results with dilepton channel, two results with lepton+jets channel and the world average are shown. Note that the result of this analysis is not shown in the plot. 

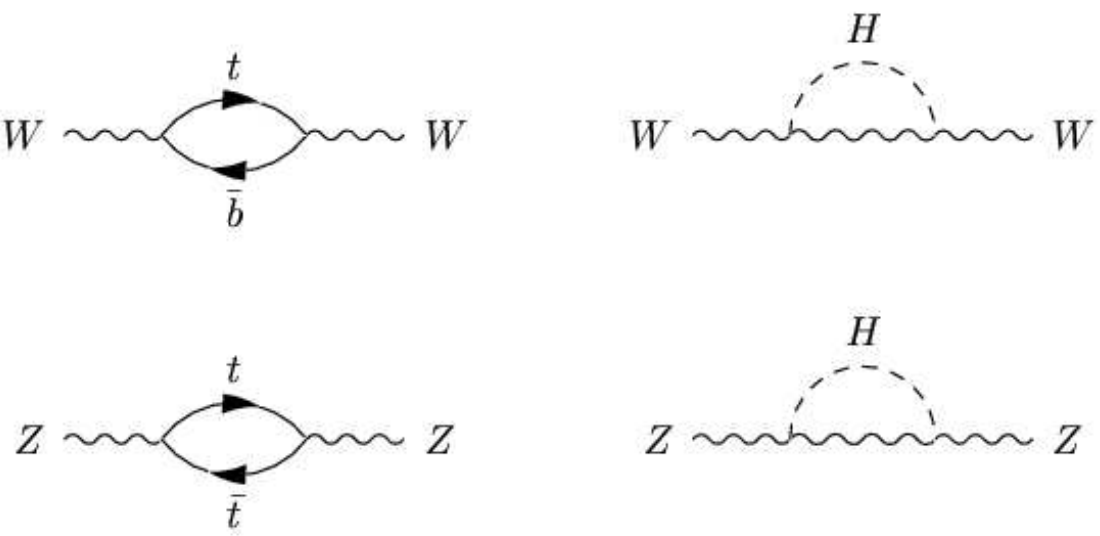

Figure 1.9: Contribution of self-coupling loops to higher order quantum corrections.

measurements, performed at LEP and by SLD, CDF, and DØ, as a function of the Higgs-boson mass, assuming the standard model to be the correct theory of nature. The preferred value for its mass, corresponding to the minimum of the curve, is at $114 \mathrm{GeV}$, with an experimental uncertainty of +69 and $-45 \mathrm{GeV}$ [18] (at 68 percent confidence level derived from $\Delta \chi^{2}=1$ for the black line, thus not taking the theoretical uncertainty shown as the blue band into account). This result is only very little affected by the low- $Q^{2}$ results.

While this is not a proof that the standard model Higgs boson actually exists, it does serve as a guideline in what mass range to look for it. The precision electroweak measurements tell us that the mass of the standard model Higgs boson is lower than about $260 \mathrm{GeV}$ [18] (one-sided 95 percent confidence level upper limit derived from $\Delta \chi^{2}=2.7$ for the blue band, thus including both the experimental and the theoretical uncertainty).

Of course, the Higgs boson is also searched for directly. Only the direct observation of the Higgs boson constitutes a proof of its existence. The LEP Higgs Working Group (LEP HWG) [19] combines the results on the direct searches for the Higgs boson at LEP. These direct searches lead to the conclusion that the standard model Higgs boson must be heavier than $114.4 \mathrm{GeV}$ (95 percent confidence level limit), as indicated by the excluded area drawn in yellow in Figure 1.11 , which is well compatible with the above prediction. Thus, the mass of the standard model Higgs boson is now restricted to a small range of values by the data. In fact, toward the end of the LEP running in the year 2000, tantalizing hints for the direct observation of the Higgs signal, corresponding to a Higgs boson mass around $116 \mathrm{GeV}$, have been detected; a mass value well compatible with the constraints derived from the precision electroweak measurements. 


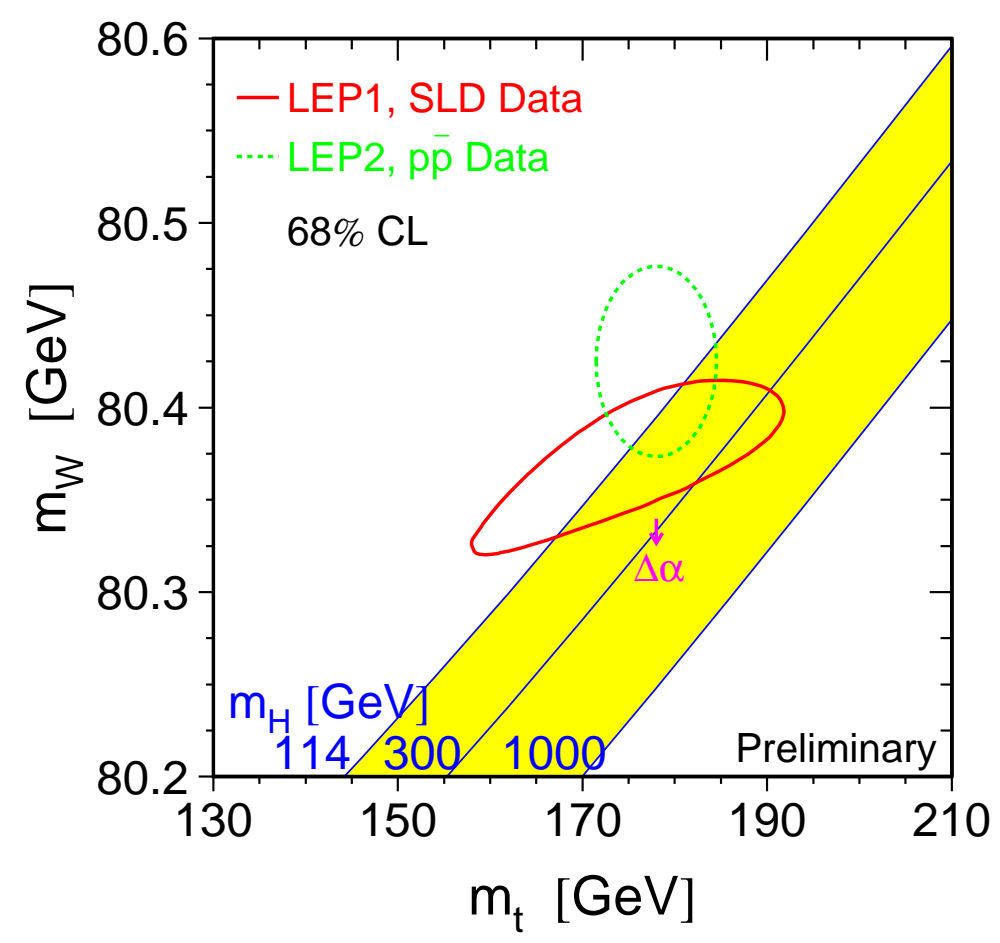

Figure 1.10: The comparison of the indirect measurements of $M_{W}$ and $M_{t o p}$ (solid contour) and the direct measurements ( $p \bar{p}$ colliders and LEP-II data) (dashed contour). In both cases the $68 \%$ C.L. contours are plotted. Also shown in the Standard Model relationship for the masses as a function of the Higgs mass. The arrow labeled $\Delta \alpha$ shows the variation of this relation if $\alpha\left(M_{Z}^{2}\right)$ is changed by one standard deviation. This variation gives an additional uncertainty to the Standard Model 


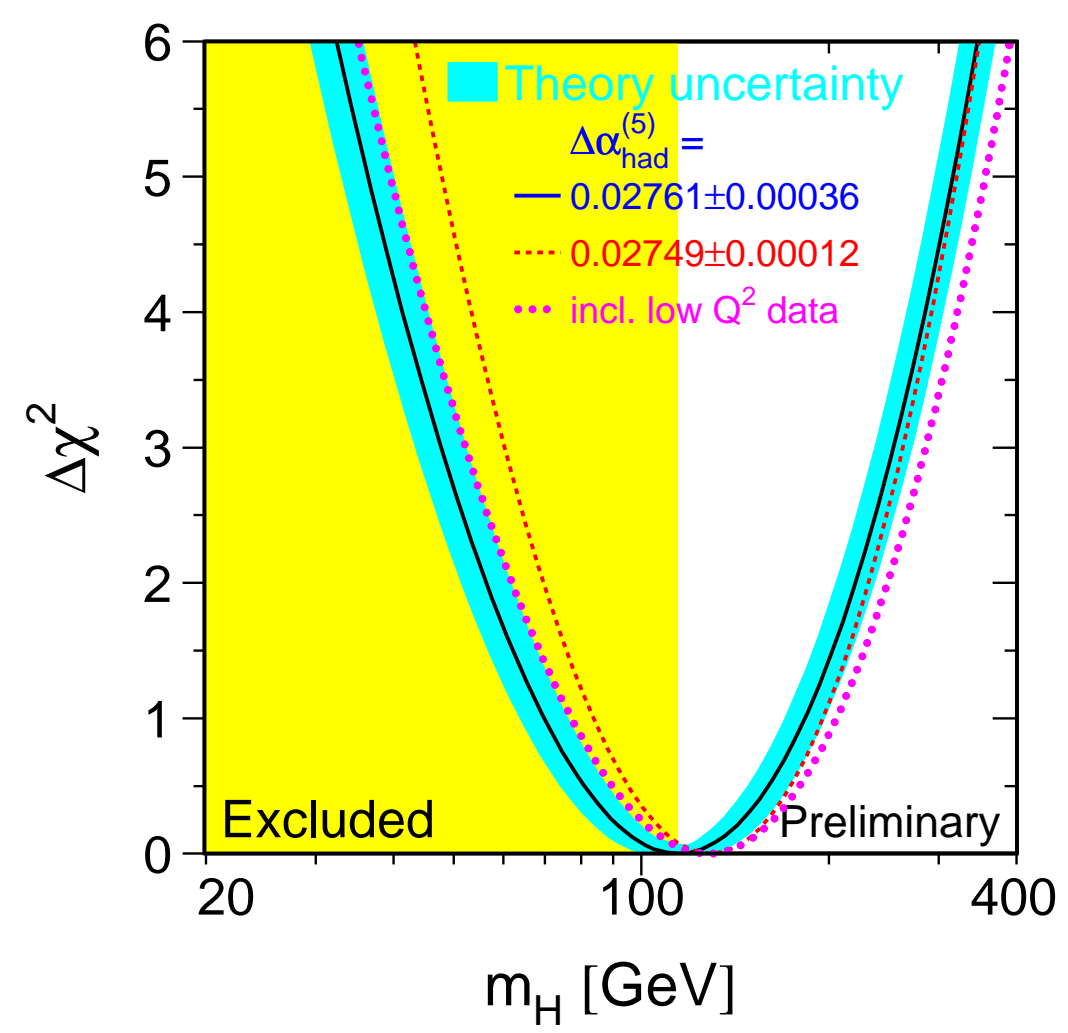

Figure 1.11: $\Delta \chi^{2}=\chi^{2}-\chi_{\min }^{2}$ vs Higgs boson mass $M_{H}$. The line is the result of the fit. The band represents an estimate of the theoretical error due to missing higher order corrections. The vertical band shows the $95 \%$ C.L. exclusion limit on $M_{H}$ from the direct search. The dashed curve is the result obtained using the evaluation of $\Delta \alpha_{h a d}^{(5)}\left(M_{Z}^{2}\right)$ from [20] 


\section{Chapter 2}

\section{Experimental Design}

\subsection{The Accelerator Complex}

In the next decade, CDF plans to carry out precise analyses of several rare physical processes whose cross section is several orders of magnitude smaller than the inelastic $p \bar{p}$ cross section. In order to obtain sufficiently large samples, several steps have been taken:

- Increase the center-of-mass energy

- Increase the luminosity

- Increase the detector's acceptance

The first two steps, and the partial reconstruction of the Tevatron which they implied, are the topic of this section.

As was stated above, the Run II proton-antiproton center of mass energy has increased to $1.96 \mathrm{TeV}$ from the Run I value of $1.8 \mathrm{TeV}$. This change provides a major increase in the reconstructed sample size; for example, the cross section for associated $t \bar{t}$ production grows by $40 \%$ with respect to Run I.

Another way to obtain a larger sample is to increase the accelerator's luminosity. In the ideal case, where the proton and antiproton beams collide head-on without a crossing angle and with optimal alignment, the Tevatron's luminosity is given by the formula

$$
\mathcal{L}=\frac{f B N_{p} N_{\bar{p}}}{2 \pi\left(\sigma_{p}^{2}+\sigma_{\bar{p}}^{2}\right)} F\left(\frac{\sigma_{l}}{\beta^{*}}\right)
$$


where $f$ is the revolution frequency, B the number of bunches in each beam, $N_{p}$ and $N_{\bar{p}}$ the number of protons and anti-protons per bunch, $\sigma_{p}$ and $\sigma_{\bar{p}}$ the transverse beam sizes (RMS) at the interaction point, and $F$ a form factor that depends on the ratio between the bunch longitudinal RMS size, $\sigma_{l}$, and the beta function ${ }^{1}$ at the interaction point, $\beta^{*}$.

\begin{tabular}{|c|c|c|c|}
\hline \hline Run & 1989 & IA (1992-93) & IB (1993-95) \\
\hline$p /$ bunch & $7.00 \mathrm{E}+10$ & $1.20 \mathrm{E}+11$ & $2.32 \mathrm{E}+11$ \\
$\bar{p} /$ bunch & $2.90 \mathrm{E}+10$ & $3.10 \mathrm{E}+10$ & $5.50 \mathrm{E}+10$ \\
$p$ emittance (mm mrad) & 25 & 20 & 23 \\
$\bar{p}$ emittance (mm mrad) & 18 & 12 & 13 \\
Beta @@ IP (m) & 0.55 & 0.35 & 0.35 \\
Energy (GeV/particle) & 900 & 900 & 900 \\
Bunches & 6 & 6 & 6 \\
Bunch length (rms, m) & 0.65 & 0.55 & 0.6 \\
Form Factor & 0.71 & 0.62 & 0.59 \\
Typical $\mathcal{L}$ (cm $\left.{ }^{-2} \mathrm{~s}^{-1}\right)$ & $1.60 \mathrm{E}+30$ & $5.42 \mathrm{E}+30$ & $1.58 \mathrm{E}+31$ \\
Best $\mathcal{L}\left(\mathrm{cm}^{-2} \mathrm{~s}^{-1}\right)$ & $2.05 \mathrm{E}+30$ & $9.22 \mathrm{E}+30$ & $2.50 \mathrm{E}+31$ \\
$\int \mathcal{L} d t$ (pb $^{-1} /$ week) & 0.32 & 1.09 & 3.18 \\
Bunch Spacing (nsec) & 3500 & 3500 & 3500 \\
Interactions/crossing & 0.25 & 0.85 & 2.48 \\
What's New? & & Separators & Linac Upgrade \\
\hline \hline
\end{tabular}

Table 2.1: Evolution of Tevatron parameters. "Typical" luminosity is quoted at the beginning of a store.

As shown in Table 2.1 and Table 2.2, the most significant improvements in luminosity are obtained by increasing the number of bunches per beam from 6 to 36 , and then to 108, while keeping the number of particles per bunch similar to or higher than the Run I figure.

A limiting factor in the choice of accelerator parameters is the superposition of multiple elementary proton-antiproton interactions within the same bunch crossing. At high luminosities, this superposition increases the complexity of the event, making its reconstruction more difficult.

\footnotetext{
${ }^{1}$ Supposing the profile of the beam in the phase space $\left(x, x^{\prime}\right)$ is an ellipse of semi-axes $\sigma$ and $\sigma^{\prime}$, the amplitude function $\beta$ is defined as the ratio $\sigma / \sigma^{\prime}$, while the beam emittance is the phase volume $\epsilon=\pi \sigma \sigma^{\prime}$.
} 


\begin{tabular}{|c|c|}
\hline \hline Run & II (2001- ) \\
\hline$p /$ bunch & $3.30 \mathrm{E}+11$ \\
$\bar{p} /$ bunch & $3.60 \mathrm{E}+10$ \\
$p$ emittance (mm mrad) & 30 \\
$\bar{p}$ emittance (mm mrad) & 20 \\
Beta @@ IP (m) & 0.35 \\
Energy (GeV/particle) & 980 \\
Bunches & 36 \\
Bunch length (rms, m) & 0.43 \\
Form factor & 0.70 \\
Typical $\mathcal{L}$ (cm $\left.{ }^{-2} \mathrm{~s}^{-1}\right)$ & $4-10 \mathrm{E}+31$ \\
$\int \mathcal{L} d t$ (pb ${ }^{-1} /$ week $)$ & 8 \\
Bunch Spacing (nsec) & 396 \\
Interactions/crossing & 2.17 \\
What's New? $^{\prime}$ & $\bar{p}$ improvements \\
\hline \hline
\end{tabular}

Table 2.2: Evolution of Tevatron parameters. "Typical" luminosity is quoted at the beginning of a store. 
Production and acceleration of protons and anti-protons at Fermilab requires a chain of accelerators, each boosting particles to higher energies. Each step will be described in the following pages.

\subsubsection{Proton production and boosting}

The process begins with a Cockcroft-Walton accelerator, which feeds negative hydrogen ions to a $150 \mathrm{~m}$ linear accelerator. The Linac itself was upgraded in 1993, increasing its energy from $200 \mathrm{MeV}$ to $400 \mathrm{MeV}$; this made it possible, during Run Ib, to double the number of protons per bunch, and to increase by about $50 \%$ the production rate of antiprotons.

After being stripped of electrons, the protons enter the Booster, a synchrotron whose diameter is about $150 \mathrm{~m}$, where they reach a kinetic energy of $8 \mathrm{GeV}$. Together, Linac and Booster are able to provide pulses of $5 \cdot 10^{12}$ protons for antiproton production every $1.5 \mathrm{~s}$, or $6 \cdot 10^{10}$ protons per bunch in series of 5 to 7 bunches, repeated 36 times every four seconds.

After leaving the Booster, protons are transferred to the Main Injector, a newly built circular accelerator that replaced the older Main Ring.

\subsubsection{Main Injector}

The Main Ring was originally built to provide $400 \mathrm{GeV}$ protons to Fermilab's fixed target experiments; later on, it was converted to act as an injector to the Tevatron. The new operational requirements for the Main Ring did not match its original design; therefore, during Run I, the Main Ring was a performance bottleneck. To quote an example, the Main Ring was never able to make full use of the Booster's capabilities: the Main Ring's aperture $(12 \pi \mathrm{mm} \mathrm{mrad})^{2}$ is only $60 \%$ of the Booster's aperture $(20 \pi \mathrm{mm}$ mrad). The situation would be even worse in Run II, with the Booster's aperture at injection increasing to $30 \pi \mathrm{mm}-\mathrm{mrad}$.

The Main Injector was designed to solve this problem, while providing further benefits. It is a $3-\mathrm{km}$ circular accelerator, which brings protons and anti-protons from a kinetic energy of 8 $\mathrm{GeV}$ to a total energy of up to $150 \mathrm{GeV}$. Its transverse admittance is larger than $40 \pi \mathrm{mm} \mathrm{mrad}$, more than enough to accommodate particle bunches from the Booster; its emittance is about $12 \pi \mathrm{mm}$ mrad. The maximum beam size is $3 \cdot 10^{13}$ particles, divided into up to 504 bunches of $6 \cdot 10^{10}$ (anti)protons.

\footnotetext{
${ }^{2}$ All emittances are normalized at $95 \%$ of the beam.
} 
Being more flexible than the Main Ring, the Main Injector can be used in several operation modes:

- Antiproton production;

- Proton and antiproton boosting, before injection into the Tevatron in collider mode;

- Antiproton deceleration, in order to recover unused anti-protons after a Tevatron collision run;

- Proton and antiproton acceleration for fixed target experiments, either directly or as a booster for the Tevatron.

\subsubsection{Antiproton production}

In order to produce anti-protons, a pulse of $5 \cdot 10^{12}$ protons at $120 \mathrm{GeV}$ is extracted from the Main Injector and focused on a nickel target. A lithium lens collects the anti-protons produced by the collision, with a wide acceptance around the forward direction, at energies close to $8 \mathrm{GeV}$. The antiproton bunches are then moved to a Debuncher Ring, where they are transformed into a continuous beam and stochastically cooled, and then to the Accumulator, where they are further cooled. The antiproton stacking rate during Run I was about $7 \cdot 10^{10} \bar{p}$ /hour; Run II upgrades, ranging from antiproton cooling to improving the lithium lens, increases this rate by a factor of three to four.

When a sufficient number of anti-protons (up to $10^{12}$ ) is available, stacking is suspended; the anti-protons are further cooled, and then transferred, with an aperture of $10 \pi \mathrm{mm}$ mrad and a $\Delta p / p<10^{-3}$, to the antiproton Recycler Ring.

\subsubsection{Recycler Ring}

The Recycler Ring lies in the same enclosure as the Main Injector; contrarily to the other rings at Fermilab, it is built with permanent magnets. During Run I, the antiproton accumulation ring was found to suffer some kind of failure approximately once a week; this led to the loss of the entire store. Permanent magnets, not being prone to the most common causes of failure (such as power loss and lightning) provide a very stable repository for up to $3 \cdot 10^{12}$ anti-protons at a time. 
During Run II, bunches of $2 \cdot 10^{11}$ recently produced anti-protons are transferred from the Accumulator to the Recycler Ring every about half an hour, thus keeping the total beam current in the Accumulator small (below $10 \mathrm{~mA}$, compared to the $200 \mathrm{~mA}$ antiproton current in Run I).

Antiproton production is one of the limiting factors in the efficiency of Fermilab's colliders. At the end of a store, $75 \%$ of the antiprotons are expected to be still circulating in the Tevatron; by recycling $2 / 3$ of these anti-protons, the average luminosity can be increased by a factor of two.

\subsubsection{Tevatron}

The Tevatron is about $6-\mathrm{km}$ circular accelerator, where protons and anti-protons, rotating in opposite directions inside the same beam pipe, are accelerated from $150 \mathrm{GeV}$ to $1 \mathrm{TeV}$. Making use of the upgrades in the rest of the accelerator chain, the Tevatron can provide an initial luminosity of $5 \cdot 10^{31} \mathrm{~cm}^{2} \mathrm{~s}^{-1}$.

During a collider store, instant luminosity slowly decreases. In the early stages of the store, the most important cause for this decrease is intrabeam scattering; some hours later, the depletion of anti-protons during collisions becomes more relevant. Luminosity is expected to decrease to $50 \%$ in about seven hours, and to $1 / e$ in twelve hours. After a typical store duration of eight hours, $75 \%$ of the antiprotons are still available; they are decelerated in the Tevatron and in the Main Injector, and then stored in the Recycler Ring and re-cooled Recycler is not used for the current $\bar{p} p$ collisions.

The Tevatron can also be used in fixed-target mode: it can accelerate up to $3 \cdot 10^{13}$ protons at a time to an energy of $800 \mathrm{GeV}$, and deliver single bunches to be used in proton, meson and neutrino experiments.

Other operational parameters of the Tevatron are listed in Table 2.1 and Table 2.2.

\subsubsection{Beam monitors}

Operation of colliders at the Tevatron requires a constant monitoring of the beam position and luminosity. From a conceptual point of view, this is done in Run II as it was done in Run I.

The luminosity monitor consists in two arrays of scintillators, placed on both sides of the interaction region. A coincidence of particles moving away from the interaction point, both in the $p$ and $\bar{p}$ direction, is interpreted as a contribution to luminosity; bunches of particles moving in a single direction, without a coincident bunch in the opposite direction, are flagged as beam 
losses.

The beam position, on the other hand, is measured by the collider detectors themselves. During Run I, the detector was able to locate the beam within $5 \mu \mathrm{m}$ in about five minutes; other beam parameters, such as slope and transverse profile, were calculated over longer time intervals (about two hours). In Run II, the same operations are performed more quickly.

\subsection{The CDF Detector}

As stated above, one of the aims of Run II is to reconstruct and store a large sample of rare events. To achieve this result, the number of bunches in each beam increased first by a factor of six with respect to Run I. An immediate consequence is that the time between two successive interactions decreased by the same factor. Several parts of the detectors have been rebuilt from scratch in order to accommodate the higher collision rate.

While the detector was redesigned, efforts were also made to extend its acceptance. The geometrical coverage was increased, by adding new detector elements or enlarging the previously existing ones; the trigger system became able to detect some interesting event features at an earlier stage than in Run I, thus improving the signal to background ratio.

As shown in figure 2.1, the tracking system of CDF II is placed inside a superconducting solenoid, while calorimeter and muon systems are outside the magnet. The rest of this chapter will provide a short description of the detector subsystems, with an emphasis on the upgrades since Run I. A complete description of CDF can be found in [21].

In the standard CDF geometry, the $\hat{z}$ axis is oriented along the axis of the solenoid, the $\hat{x}$ axis points away from the center of the Tevatron, and the $\hat{y}$ axis points up. The origin is at the interaction point. The polar angle $\theta$ is measured starting from the positive $\hat{z}$ axis; the rapidity $y$ is defined by

$$
y=\frac{1}{2} \ln \left(\frac{E+p_{z}}{E-p_{z}}\right)
$$

For the high energy particles, $E \sim p$ and $p_{z}=p \cos \theta$, the rapidity is nearly equal to the pseudorapidity defined as

$$
\eta=-\ln \left(\tan \frac{\theta}{2}\right)
$$

In hadron-hadron collisions, a rapidity $y$ (or pseudorapidity $\eta$ ), a transverse momentum $p_{T}$ 
and an azimuth angle $\phi$ are usually used. The invariant cross section is written as

$$
E \frac{d^{3} \sigma}{d^{3} p}=\frac{d^{3} \sigma}{d \phi d y p_{T} d p_{T}} \rightarrow \frac{d^{3} \sigma}{\pi d y d p_{T}^{2}}
$$

The second form is obtained using the identity $d y / d p_{z}=1 / E$, and the third form represents the average over $\phi$. The total multiplicity of particles in collisions is given by $d \sigma / d y$ and this means that the multiplicity is flat in $\eta$.

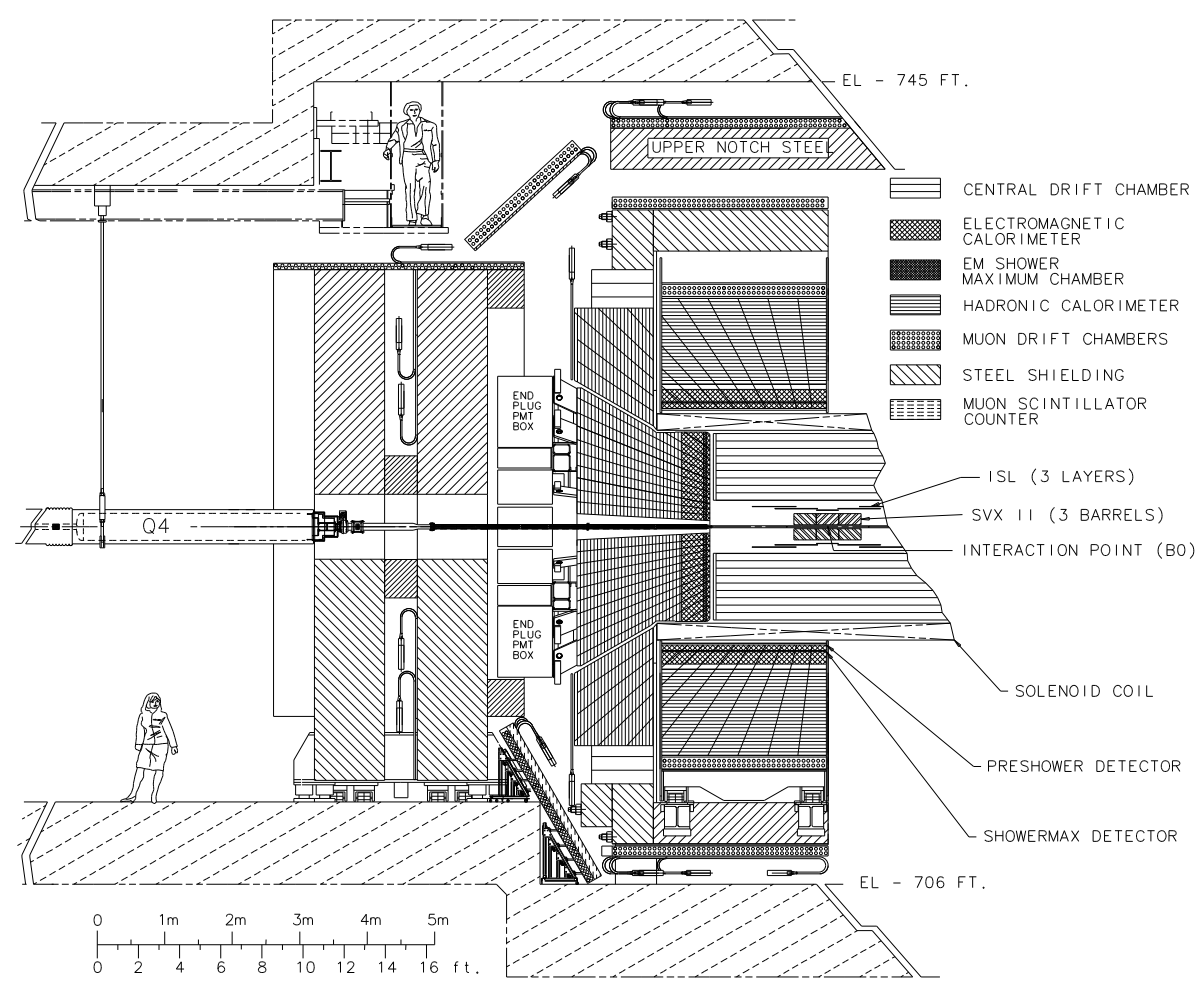

Figure 2.1: Elevation view of one half of the CDF II detector

\subsection{Tracking system}

The innermost parts of the CDF II detector are devoted to tracking charged particles.

\subsubsection{Silicon Vertex Detector}

CDF II makes use of three concentric silicon detectors: "Layer 00" (L00), the Silicon Vertex Detector (SVX II, or SVX in short), and the Intermediate Silicon Layers (ISL) [23]. 
SVX II is the Run II baseline detector. It consists of five layers of double-sided silicon wafers. One side of each wafer provides measurements in the transverse plane (axial strips); the other side's strips deliver 3D information. SVX II extends radially from 2.5 to $10 \mathrm{~cm}$, and along $z$ up to $45 \mathrm{~cm}$ on either side of the interaction point.

The ISL consists of a double-sided silicon layer, similar to those in SVX II, placed at $r=$ $22 \mathrm{~cm}$ in the central $\eta$ region, and of two forward layers $(1<|\eta|<2)$ respectively at 20 and $29 \mathrm{~cm}$ from the beam line. Together with SVX II, the ISL makes it possible to reconstruct tracks in the forward region, which lies beyond the acceptance region of the outer tracker.

Layer 00 is the most recent addition to the CDF II tracker. It is a single sided, radiationhard silicon layer, placed immediately outside the beam pipe, at $r \simeq 1.5 \mathrm{~cm}$. Being so close to the interaction point, Layer 00 improves noticeably the impact parameter resolution. In case the innermost SVX II layer suffers from radiation damage during Run II, Layer 00 also acts as a backup.

Compared to the shorter, 4-layer, single-sided vertex detector of Run I, the new silicon tracker provides a much wider acceptance, better resolution, three-dimensional reconstruction, and can be used in stand-alone mode, without input from the Central Outer Tracker (described hereafter).

\subsubsection{Central Outer Tracker}

Outside the silicon detector, at a distance between 40 and $138 \mathrm{~cm}$ from the beam, lies the Central Outer Tracker [24]. It is a new open-cell drift chamber, able to reconstruct tracks in the $|\eta|<1$ region. The COT replaces an older drift chamber, the CTC [25], that would have been unable to cope with the expected occupancy and event rate of Run II.

Each of the eight superlayers of cells consists of twelve layers of sense wires, alternating with field-shaping wires. Axial superlayers alternate with stereo superlayers, thus providing 48 axial and 48 stereo measurements for each track.

In the COT, the cell size is roughly four times smaller than in the CTC. Usage of a faster gas ( $\mathrm{Ar}-$ Ethane $-\mathrm{CF}_{4}$ instead of $\mathrm{Ar}-$ Ethane) reduces the maximum drift time by a further factor of two, down to $100 \mathrm{~ns}$. This makes the COT immune from event pile-up, even at the highest collision rate of $1 /(132 \mathrm{~ns})$. 


\subsubsection{Time of flight}

A recent addition to CDF II, the time-of-flight detector is an array of scintillator bars, placed at the outer edge of the COT, at a radial coordinate of $140 \mathrm{~cm}$. An accurate measurement of a particle's time of flight in the CDF tracking volume can be used quite effectively in particle identification.

Scintillator bars are about three meters long, matching the COT active volume; their thickness $(4 \mathrm{~cm})$ is limited by the space which remained available between the previously designed COT and magnet. Their width was determined by occupancy ${ }^{3}$ and resolution considerations; the best choice turned out to be also of the order of $4 \mathrm{~cm}$. The bars have a trapezoidal cross section, in order to minimize cracks in the geometry; the scintillating material is Bicron 408, which has a short rise time and a long $(380 \mathrm{~cm})$ attenuation length.

Photomultiplier tubes, attached to both ends of each bar, provide time and pulse height measurements. By comparing the two pairs of results, the detector determines the instant in which a particle crossed the scintillator with an accuracy of about $100 \mathrm{ps}$, and the $z$ coordinate of the intersection. The latter measurement is compared to the results of $3 \mathrm{D}$ track reconstruction in the inner tracking volume, to associate a time of flight to each track.

\subsubsection{Magnet}

The CDF tracking systems are enclosed in a superconducting solenoid, which provides a uniform magnetic field of up to $1.5 \mathrm{~T}$ along the detector axis, over a cylindrical fiducial volume $3.5 \mathrm{~m}$ long and $2.8 \mathrm{~m}$ in diameter.

The solenoid is built of an Al-stabilized NbTi superconductor, able to withstand currents up to $5000 \mathrm{~A}$, and operating at liquid helium temperature. During most of Run I, the magnet operated at $4650 \mathrm{~A}$, corresponding to a current density of $1115 \mathrm{~A} / \mathrm{m}$ and a central field of $1.41 \mathrm{~T}$.

Although the design lifetime of the solenoid was only ten years, it is possible to reuse the magnet during Run II. The cool-down procedures that were used during Run I limited mechanical stress to the coil, avoiding fatigue damage.

\footnotetext{
${ }^{3}$ Detector occupancy depends on the average number of superimposed interactions, which increases with luminosity. TOF occupancy is estimated to be 0.1 with 2 superimposed events, and 0.4 with 10 events.
} 


\subsection{Calorimetry}

\subsubsection{Overview}

CDF uses scintillator sampling calorimeters, divided into separate electromagnetic and hadronic sections, and providing coverage for $|\eta| \leq 3.64$. The calorimeter was an essential tool in selection and reconstruction of events in Run I; in Run II it continues to measure the energy of photons, electrons, jets, and the missing transverse energy ${ }^{4}$ associated to neutrinos and possibly to neutral exotic particles.

Calorimeter calibration can be performed by matching the tracks found in the tracking system to the corresponding calorimetry towers; during Run I, this provided a $2.5 \%$ accuracy on jet energy measurements.

\begin{tabular}{c|c|c}
\hline \hline$|\eta|$ range & $\Delta \phi$ & $\Delta \eta$ \\
\hline $0-1.1(1.2 \mathrm{had})$ & $15^{\circ}$ & 0.1 \\
$1.1(1.2 \mathrm{had})-1.8$ & $7.5^{\circ}$ & 0.1 \\
$1.8-2.1$ & $7.5^{\circ}$ & 0.16 \\
$2.1-3.64$ & $15^{\circ}$ & $0.2-0.6$ \\
\hline \hline
\end{tabular}

Table 2.3: Calorimeter segmentation

The entire calorimeter is segmented into projective towers, whose geometry is summarized in table 2.3. Each tower consists of alternating layers of passive material (lead for the e.m. section, iron for the hadronic compartment) and scintillator tiles. The signal is read via wavelength shifters (WLS) embedded in the scintillator; light from the WLS is then carried to photomultiplier tubes. Table 2.4 shows the most important characteristics of each calorimeter sector. The central and end-wall calorimeters $(|\eta|<1.1)[26]$ [27] were recycled from Run I; the plug ones $(1.1<|\eta|<3.64)$ were built anew, to replace an older gas calorimeter that would not be able to function at the increased event rate of Run II.

\subsubsection{Central calorimeter}

Apart from the electronics, the central calorimeter in CDF Run II is the same used during Run I. The energy measurement response time is already fast enough to accommodate a $132 \mathrm{~ns}$ bunch spacing.

\footnotetext{
${ }^{4}$ Contrarily to $e^{+} e^{-}$colliders, in $p \bar{p}$ colliders the longitudinal momentum of the initial state is unknown.
} 


\begin{tabular}{|c|c|c|}
\hline \hline & Central and End-wall & Plug \\
\hline Electromagnetic: & & \\
Thickness & $19 X_{0}, 1 \lambda$ & $21 X_{0}, 1 \lambda$ \\
- per sample $(\mathrm{Pb})$ & $0.6 X_{0}$ & $0.8 X_{0}$ \\
- per sample (scint.) & $5 \mathrm{~mm}$ & $4.5 \mathrm{~mm}$ \\
Light yield & 160 p.e. $/ \mathrm{GeV}$ & 300 p.e. $/ \mathrm{GeV}$ \\
Sampling resolution & $11.6 \% / \sqrt{E}$ & $14 \% / \sqrt{E}$ \\
Stochastic resolution & $14 \% / \sqrt{E}$ & $16 \% / \sqrt{E}$ \\
\hline Hadronic: & & $7 \lambda$ \\
Thickness & 1 in (central) & $2 \mathrm{in}$ \\
- per sample (Fe) & 2 in (end-wall) & $6 \mathrm{~mm}$ \\
per sample (scint.) & $6 \mathrm{~mm}$ & 39 p.e./GeV \\
Light yield & 40 p.e./GeV & $80 \% / \sqrt{E} \oplus 5 \%$ \\
Resolution & $75 \% / \sqrt{E} \oplus 3 \%$ &
\end{tabular}

Table 2.4: Characteristics of the CDF II calorimeter 


\section{Central electromagnetic calorimeter}

The central electromagnetic calorimeter consists of projective towers of alternating lead and scintillator. The signal is read via a $\mathrm{PMMA}^{5}$ wavelength shifter, and carried via clear fiber to photomultiplier tubes. None of these is expected to suffer much from radiation damage. The light yield loss is expected to be around $1 \%$ per year; $60 \%$ of this loss is explained by the gradual shortening of the attenuation length in the scintillator.

A two-dimensional wire chamber is embedded in the calorimeter, as a shower maximum detector (CES). Its usage in the Run I trigger decreased the fake electron trigger rate by a factor of two [28].

Another wire chamber is placed immediately in front of the calorimeter, to act as a preshower detector (CPR) which uses the tracker and the solenoid coil as radiators. The CPR has proven to be extremely useful in rejection of electron background; it also reduced systematic uncertainties for direct photon measurements by a factor of three [29].

\section{Central hadronic calorimeter}

The central and end-wall hadronic calorimeters use 23 iron layers as radiator. The scintillator should not suffer radiation damage from measured events.

The hadronic compartment geometry matches the projective towers of the electromagnetic calorimeter.

\subsubsection{Plug calorimeter upgrade}

The CDF II plug calorimeter, shown in figure 2.2, covers the $\eta$ region between 1.1 and 3.64, corresponding to polar angles between $37^{\circ}$ and $3^{\circ}$. It replaces an older gas calorimeter, whose response speed was too slow for usage at the CDF II $132 \mathrm{~ns}$ interbunch. Being based on the same principles as the central calorimeter, the new plug calorimeter also makes experimental data more homogeneous.

The calorimeter is divided in 12 concentric $\eta$ regions, which are further segmented in 24 (for $|\eta|<2.11$ ) or 12 (for $|\eta|>2.11$ ) projective towers.

\footnotetext{
${ }^{5} \mathrm{PMMA}=$ polymethylmethacrylate
} 


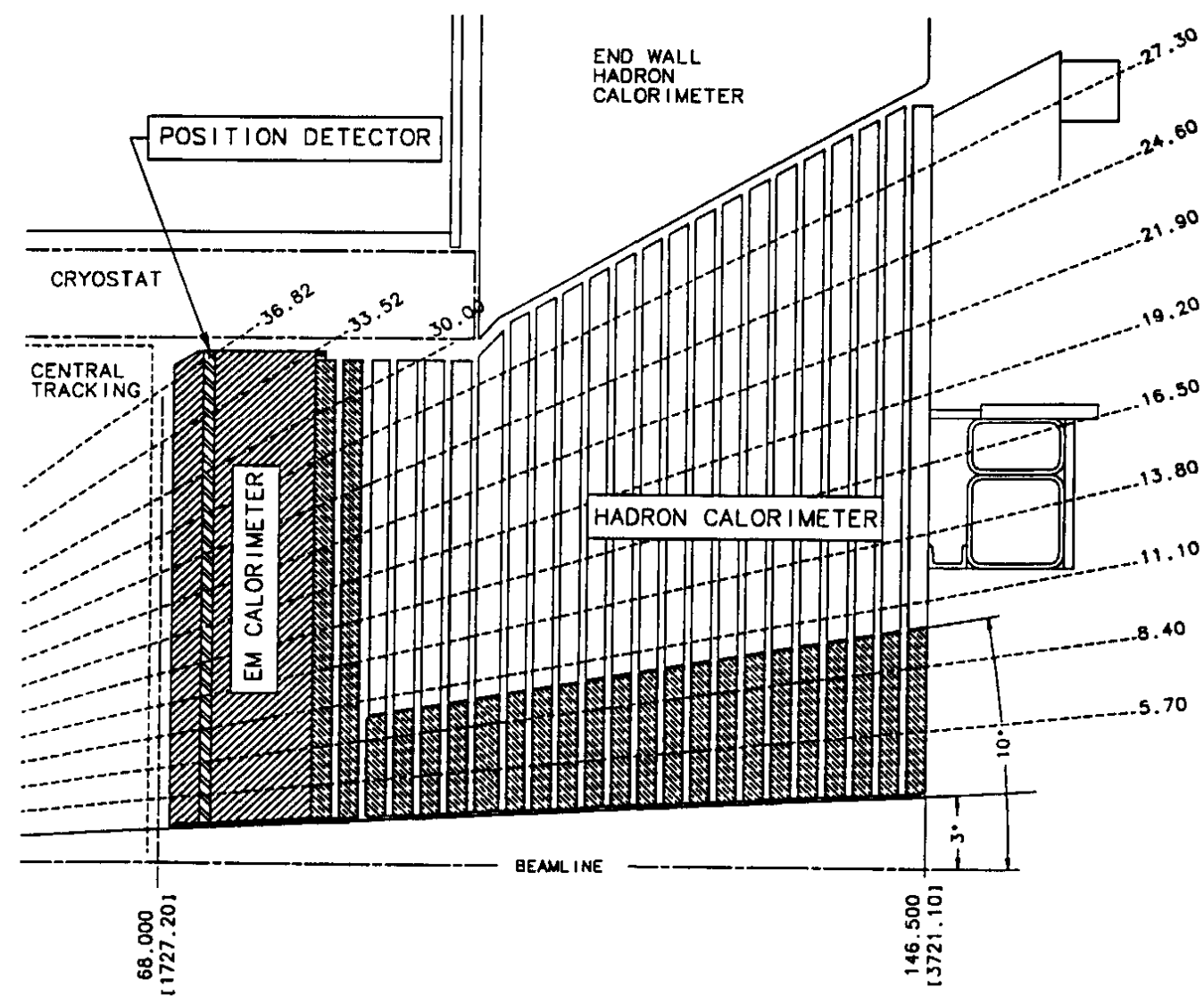

Figure 2.2: Cross section of half CDF II plug calorimeter 


\section{Plug electromagnetic calorimeter}

The EM section of the plug calorimeter consists of 23 absorber-scintillator layers. A calciumtin-lead alloy, enclosed between steel plates, is used as absorber.

The first layer of the EM section is used as a preshower detector. In order to distinguish $\gamma$ from $\pi^{0}$ reliably, the light yield needs to be higher than on other layers. Therefore, the first scintillator layer is thicker (10 $\mathrm{mm}$ instead of $6 \mathrm{~mm}$ ) and made of a brighter material; it is read out separately from the rest of the calorimeter, via multi-anode photomultiplier tubes (MAPMT).

As in the central calorimeter, a shower maximum detector (PES) is also embedded in the plug EM calorimeter, at a depth of about six radiation lengths. The PES consists of eight $45^{\circ}$ sectors, each covering six (or three) calorimetric towers in $\phi$; each sector is further segmented in two $\eta$ regions, in order to reduce occupancy. Within each region, scintillating strips are arranged on two layers, in directions parallel to either edge of the sector; this provides a two-dimensional measurement of the shower. The strips are $5 \mathrm{~mm}$ wide and $6 \mathrm{~mm}$ thick; they are read out via WLS fibers and MAPMT.

The PES is used to measure the position of electromagnetic showers with an accuracy reaching $1 \mathrm{~mm}$ for high-energy electrons, and to discriminate pions from photons and electrons.

\section{Plug hadronic calorimeter}

The hadron plug calorimeter was designed to optimize detector performance on $b$, electroweak and jet physics, and to help in muon detection by analyzing their rate of energy loss. It achieves an energy resolution of about $80 \% / \sqrt{E} \oplus 5 \%$, which is dominated by the sampling fluctuations from the steel absorber plates. The most strict requirement is that the light yield within each tile should be uniform to $4 \%$ or better; disuniformity between different tiles is not as important, as the hadron shower usually affects ten or more layers.

\subsection{Muon chambers}

The outermost component of CDF II is a set of scintillators, drift tubes and steel absorbers, used for the detection of muons.

During Run I, detection of muons has proven to be an important requirement, both for the analysis of several physics channels and for calibration. For example, a clean sample of $W$ bosons is obtained by reconstructing their muon decay mode; $J / \psi \rightarrow \mu^{+} \mu^{-}$decays are an important 
part of the heavy quark physics program, as well as a tool to measure systematic effects in the detector.

The tracking improvements from Run I to Run II have a deep impact on muon detection. Before the upgrades, muons in the central region were identified by their penetrating power, and their momentum was measured in the central tracking chamber. On the contrary, the momentum of forward muons had to be measured in the muon chambers themselves, by resorting to a toroidal magnet, as the central tracker only covered the $|\eta|<1$ region.

With the SVX II upgrade, this distinction falls: measurement of muon momentum can be performed in the central tracker, where the multiple scattering effects are smaller, and the toroidal magnets are not required any longer. Central tracks are measured in the drift chamber; forward tracks $(|\eta|>1)$ are tracked in the silicon only.

Run I central muon chambers (CMU) are reused without major changes; some upgrades which started under Run I (CMP and CSP, the Central Muon/Scintillator Upgrades; CMX and CSX, the Central Muon/Scintillator Extension) are completed; and a new set of chambers, the Intermediate Muon Detector IMU, replaces the previous Forward Muon Detectors (FMU)[30].

Due to their size, muon systems are unable to take data within the Run II interbunch interval of 400 or $132 \mathrm{~ns}$; this is not a problem, since the low occupancy of the muon chambers allows integration over multiple events. Scintillators are used to associate muon stubs to the appropriate event.

Table 2.5 summarizes the information on the muon subsystems; the following sections will describe their characteristics in deeper detail.

\subsubsection{Central muon detectors}

The first muon system built at CDF, the Central Muon Detector (CMU) [31], is a set of 144 modules, each containing four layers of four rectangular cells. It is placed just outside the central hadronic calorimeter, whose 5.5 interaction lengths absorb more than $99 \%$ of the outgoing charged hadrons.

A second set of muon chambers, the Central Muon Upgrade (CMP) [32], forms a square box around the CMU, and is shielded by an additional layer of $60 \mathrm{~cm}$ of steel. Due to the detector geometry, the $\eta$ coverage varies with azimuth as shown in figure 2.3. The CMP consists of four layers of single-wire drift tubes, staggered by half cell per layer, and operated in proportional mode. On the outer surface of the CMP lies the Central Scintillator Upgrade (CSP), a layer 


\begin{tabular}{lcccc}
\hline \hline & CMU & CMP/CSP & CMX/CSX & IMU \\
\hline$\eta$ coverage & $0-0.6$ & $0-0.6$ & $0.6-1.0$ & $1.0-1.5$ \\
\hline Drift tubes: & & & & \\
thickness & $2.68 \mathrm{~cm}$ & $2.5 \mathrm{~cm}$ & $2.5 \mathrm{~cm}$ & $2.5 \mathrm{~cm}$ \\
width & $6.35 \mathrm{~cm}$ & $15 \mathrm{~cm}$ & $15 \mathrm{~cm}$ & $8.4 \mathrm{~cm}$ \\
length & $226 \mathrm{~cm}$ & $640 \mathrm{~cm}$ & $180 \mathrm{~cm}$ & $363 \mathrm{~cm}$ \\
max drift time & $0.8 \mu \mathrm{s}$ & $1.4 \mu \mathrm{s}$ & $1.4 \mu \mathrm{s}$ & $0.8 \mu \mathrm{s}$ \\
$\#$ tubes (Run Ib) & 2304 & 864 & 1536 & - \\
$\#$ tubes (Run II) & 2304 & 1076 & 2208 & 1728 \\
\hline Scintillators: & & & & \\
thickness & N/A & $2.5 \mathrm{~cm}$ & $1.5 \mathrm{~cm}$ & $2.5 \mathrm{~cm}$ \\
width & N/A & $30 \mathrm{~cm}$ & $30-40 \mathrm{~cm}$ & $17 \mathrm{~cm}$ \\
length & N/A & $320 \mathrm{~cm}$ & $180 \mathrm{~cm}$ & $180 \mathrm{~cm}$ \\
$\#$ counters (Run Ib) & N/A & 128 & 256 & - \\
$\#$ counters (Run II) & N/A & 269 & 324 & 864 \\
\hline$\pi^{0}$ int. lengths & 5.5 & 7.8 & 6.2 & $6.2-20$ \\
Min $P_{t}$ (GeV/c) & 1.4 & 2.2 & 1.4 & $1.4-2.0$ \\
MS resol. (cm GeV) & 12 & 15 & 13 & $13-25$ \\
\hline \hline
\end{tabular}

Table 2.5: Parameters of muon detection at CDF. Pion interaction length and the limit on resolution due to multiple scattering are computed at $\theta=90^{\circ}$ in the central detectors $\mathrm{CMU}$, CMP and CSP; at $\theta=55^{\circ}$ in CMX and CSX; and on the entire $\theta$ coverage for the IMU. 


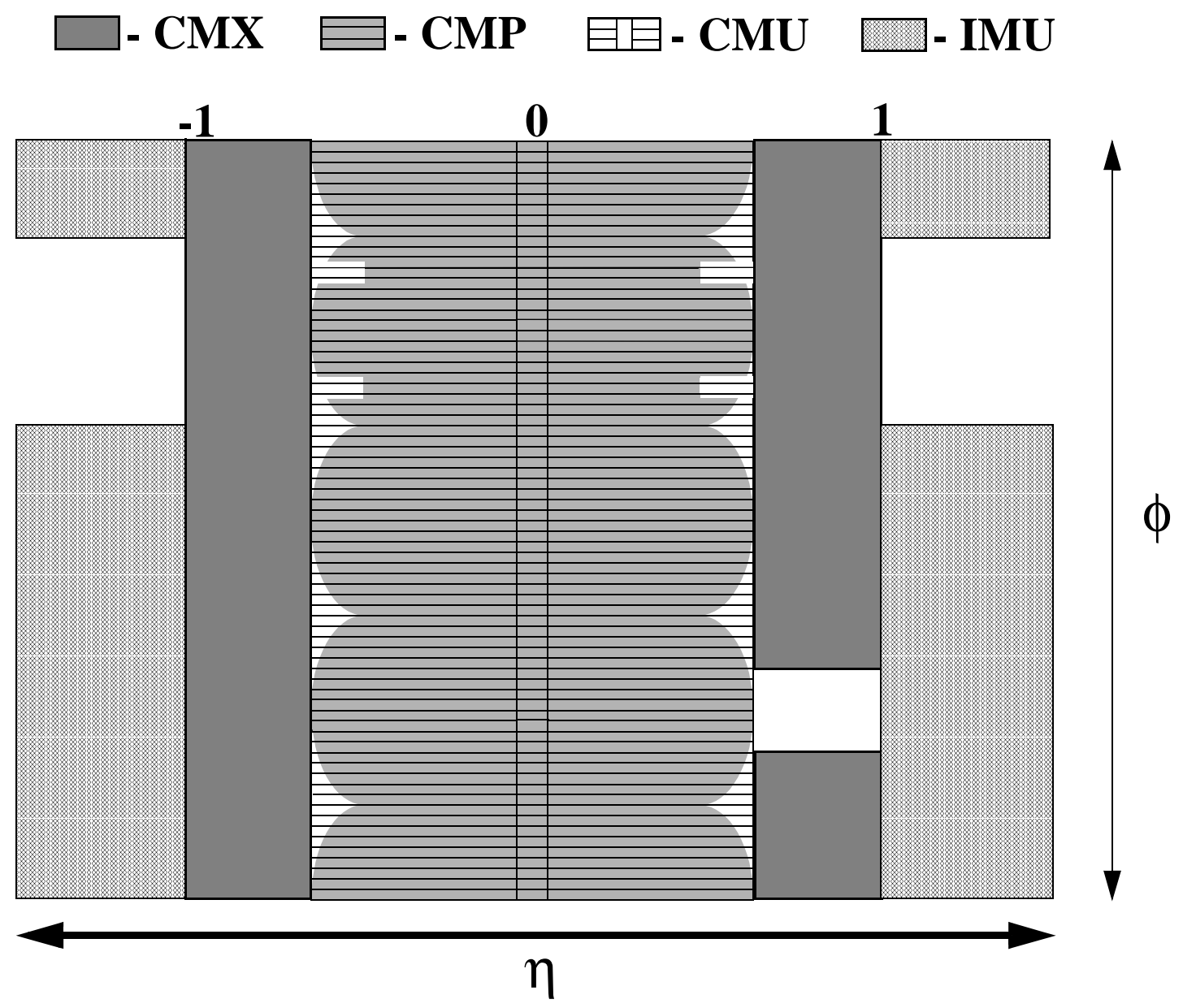

Figure 2.3: $\eta$ and $\phi$ coverage of the Run II muon systems 
of rectangular scintillator tiles.

Another upgrade which was begun in Run I is the Central Muon Extension (CMX) with the associated Central Scintillator Extension (CSX). It is a conical array of drift tubes, with scintillators on both sides; it extends the CMU/CMP $\theta$ coverage from $55^{\circ}$ to $42^{\circ}$, except in a $30^{\circ} \phi$ gap which is used by the solenoid cryogenic system.

\subsubsection{Intermediate muon detectors}

Detection of muons in the forward region is accomplished by the Intermediate Muon Detectors (IMU). This detector recycles the older Forward Muon toroidal magnets, which is moved closer to the interaction point (just outside the plug calorimeter PMT arrays). The steel toroids, together with a new pair of steel rings, act as shielding for a new array of drift tubes and scintillator counters, placed on the outer radius of the toroids.

Like the CMX/CSX, the IMU has four staggered layers of drift tubes, and two layers of scintillator. Contrarily to the CSX, one of the scintillator layers is separated from the drift tubes by a thick layer of steel; this geometry strongly suppresses fake triggers due to hadrons.

\subsection{Data Acquisition and Trigger}

Due to the increase in collision frequency, the DAQ and trigger systems of CDF had to be almost completely replaced. The new three-level architecture, schematized in figure 2.4 , is fully capable of withstanding a $132 \mathrm{~ns}$ bunch separation, while keeping dead time as short as possible.

\subsubsection{Level 1 trigger}

The front-end electronics of all detectors is fitted with a synchronous pipeline, 42 events deep, where the entire data regarding each event is stored for 5544 ns. Meanwhile, part of the data is examined in a first layer of dedicated, synchronous, highly parallel hardware processors:

- XFT, the eXtremely Fast Tracker, which reconstructs tracks on the transverse plane of the COT to propagate these tracks to the calorimeters and muon chambers;

- the Calorimeter Trigger, which detects electron and photon candidates, jets, total transverse energy, and missing transverse energy;

- the Muon Trigger, which matches XTRP tracks to stubs in the muon chambers. 


\section{Dataflow of CDF "Deadtimeless" Trigger and DAQ}

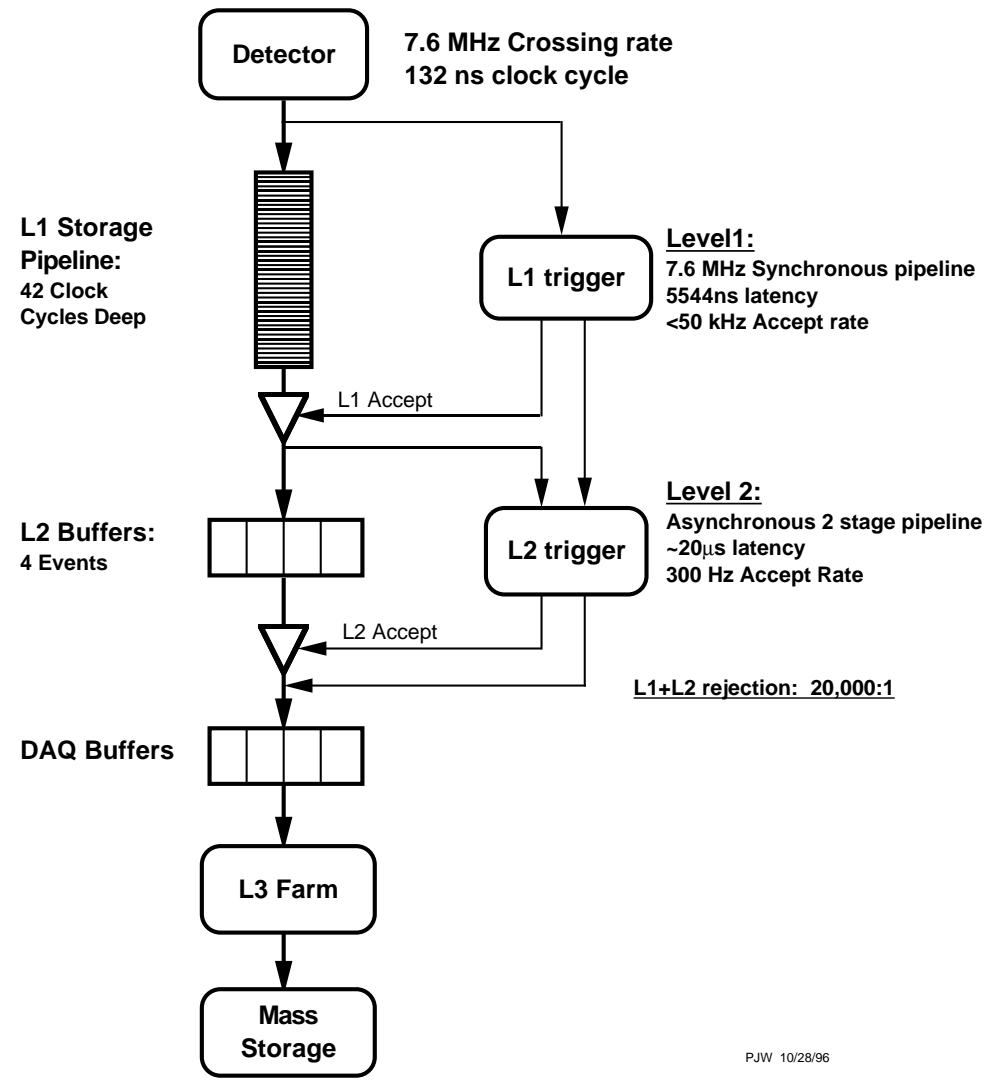

Figure 2.4: Block diagram of the CDF II Trigger 
"Objects" from the level one trigger subsystems are combined in a flexible decision module, which takes a decision by requiring the presence of a certain number of features in the event: for example, two muon candidates with $P_{t}$ above $3 \mathrm{GeV}$. Up to 64 different sets of requirements can be checked at the same time; each of these triggers can be prescaled independently of the others.

The level 1 trigger takes a decision within $4 \mu \mathrm{s}$, while the event's data is still in the pipeline. This makes the first trigger level truly deadtimeless. The rejection factor is about 150 and the event rate is about $50 \mathrm{kHz}$.

\subsubsection{Level 2 trigger}

Events matching the requirements of level 1 are downloaded into one of four asynchronous event buffers, and further analyzed by a second set of hardware processors. Trigger level 2 is asynchronous: events remain in the buffer until they are accepted or rejected. This can cause dead time, when all four buffers are full. In order to keep dead time at 10\%, with a level 1 rate of $50 \mathrm{kHz}$, level 2 has been split in two pipelined steps of $10 \mu \mathrm{s}$ each.

- Jets usually affect more than a single calorimetric tower. Calorimeter clustering (L2CAL) sums the energies collected by single towers and provides a measurement of the total jet energy.

- The calorimeter shower maximum (XCES) is used to reduce the rate of fake electrons and photons. It also makes it easier to match XFT tracks to their calorimetric clusters.

- The Silicon Vertex Tracker (SVT) reconstructs tracks in the vertex detector, measuring their impact parameter $d$. Triggering on $d$ proves extremely helpful in $b$-quark physics.

- Data is also collected from the level 1 track and muon triggers.

During the second pipelined step, the results of the first phase are fed to a set of Alpha processors; each processor examines the event for a different set of characteristics.

The level 2 accept rate is around $300 \mathrm{~Hz}$, with a rejection of about 150 .

\subsubsection{Level 3 trigger}

After being accepted by the level 2 trigger, the entire event data is read out and loaded into a Linux PC farm, where the event is fully reconstructed in software. The level 3 reconstruction program is almost fully written in $\mathrm{C}++$, using object-oriented techniques. 
After an event is reconstructed, it is sent to an event counter, where its characteristics are histogrammed; if the event passes the level 3 cuts, it is also permanently stored to tape.

Assuming a level 3 input rate of $300 \mathrm{~Hz}$, a level 3 rejection of 10, and an average event size of $250 \mathrm{kB}$.

\subsubsection{Online Monitoring}

The CDF detector consists of many detector subsystems and runs in a high rate large bandwidth data transfer environment. To take data with high efficiency and high quality, it is necessary to quickly spot problems with one of these subdetectors in real time. Multiple event monitor programs are attached to the DAQ system [33][34][35]. The online monitoring programs are called Consumers, where a consumer is defined as a process which receives events from Consumer Server Logger (CSL) in real time. CSL sends the data to the computer center where they are written to tape and forwards copies of a subset of the data to the online monitoring programs. Figure 2.5 shows a schematic view of the CDF online monitoring system (Consumer Framework). The task of Consumers is to analyze and monitor the event data and to make histograms and tables. These results could be viewed by the display browser via a server in real time. Results of the monitor are also stored as data files periodically during a run, and also archived systematically. The display browser provides a GUI to view the online monitored results, while also providing some basic utilities to do comparisons with previously stored results. By separating the two tasks of monitoring and displaying, we remove CPU bound associated with displaying graphics from the machine which runs the consumers. During the data taking, multiple consumer processes run in parallel, receiving event data with the desired trigger types from the CSL. Communication between a consumer and run control which control overall CDF DAQ system is handled by the Error Receiver. Severe errors detected by a consumer monitor program are forwarded to run control to take necessary actions. The state manager watches the state of consumers. 


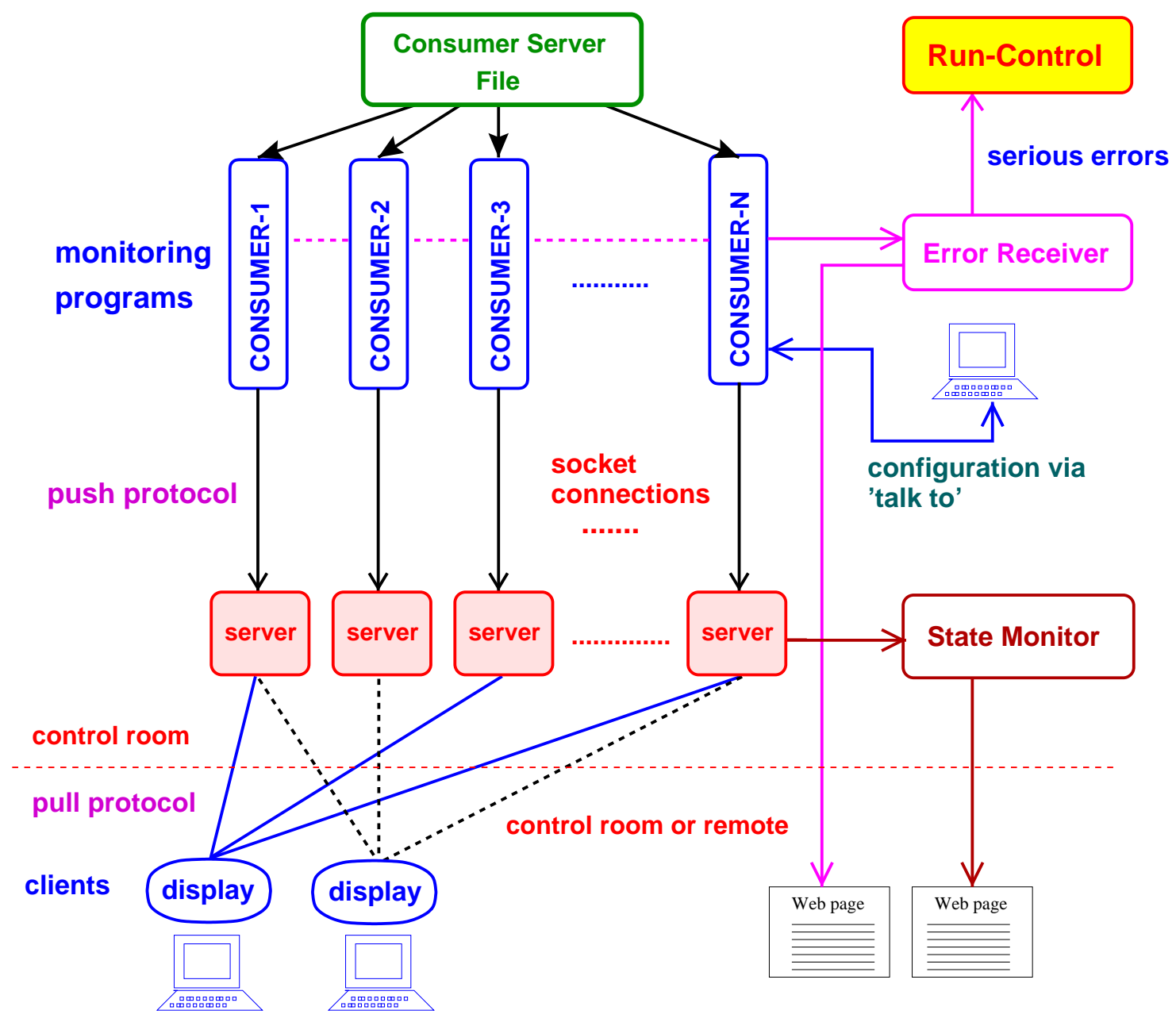

Figure 2.5: Design of the CDF online consumer framework. 


\section{Chapter 3}

\section{Particle Identifications}

\subsection{Lepton Identification}

The Identification of charged leptons produced from a $\mathrm{W}$ decay is the most important part in the selection of the $t \bar{t}$, lepton+jets sample.

After passing trigger requirement, electron candidates are identified by requiring the electrons in the central pseudo-rapidity region of the detector $(|\eta| \leq 1)$ and have EM cluster with $E_{T} \geq$ $20 \mathrm{GeV}$ and the COT track with $p_{T} \geq 10 \mathrm{GeV} / c$. Several variables are used to discriminate against charged hadrons and photon conversions. Some of them are briefly described below: We require that the extrapolated track matches the shower location as measured in the CES [21], and that the ratio of hadronic to electromagnetic energy in the calorimeter cluster, $E_{h a d} / E_{\text {em }}$ is less than 0.055 , and that the ratio of cluster energy to track momentum, $E / P$ is less than 2.0. The isolation ratio $I$, defined as the ratio of the additional energy deposits in a radius $\Delta R \equiv \sqrt{\Delta \eta^{2}+\Delta \phi^{2}}=0.4$ around the electron cluster to the electron energy, is required to be less than 0.1 . Conversions are removed by vetoing events that have a pair of tracks (one of them the electrons) which satisfies (1) Opposite sign charges, (2) the distance between the tracks in the $r-\phi$ plane where the tracks are parallel, $\Delta(x y)$ is less than $0.2 \mathrm{~cm}$, and (3) the difference between the polar angle cotangent of the two tracks, $|\cot \theta|$ is less than 0.04 . Fiducial cuts on the electromagnetic shower position in CES are required to insure that the electron is away from the calorimeter boundaries to measure the energy well enough. For isolated high momentum electrons from W decay, the tracking efficiency is measured to be $99.93{ }_{-0.35}^{+0.07 \%}$. The 
transverse energy can be measured from the electromagnetic cluster with a precision $\sigma / E_{T}=$ $13.5 \% / \sqrt{E_{T}(G e V)} \oplus 2 \%[26]$ and the transverse momentum resolution is found to be $\delta p_{T} / p_{T}$ $\approx 0.15 \% \times p_{T}(\mathrm{GeV} / c)$.

Muon candidates are identified by extrapolating the COT tracks to the muon detector. We have two types of high- $p_{T}$ muon sample used in this analysis. One is a track linked to a line(track) segment in both CMU and CMUP called a CMUP muon $(|\eta| \leq 0.6)$, and another type is a track linked to CMX called a CMX muon $(0.6 \leq|\eta| \leq 1.0)$. For both CMUP and CMX muons, we require the COT track with $p_{T} \geq 20 \mathrm{GeV} / c$, and that the energy in the calorimeter tower containing the muon is consistent with the deposit expected from a minimum ionizing particle to reject secondary particles in charged hadron showers in calorimeter which produce tracks in the muon chambers. Backgrounds from cosmic rays are removed by requiring that the distance of closest approach of the reconstructed track to the beam line, $d$ is less than $0.2 \mathrm{~cm}$. For high momentum COT tracks, the resolution at the origin is $\delta z \approx 0.5 \mathrm{~cm}$ along the beamline and $\delta d \approx 350 \mu \mathrm{m}$ for the impact parameter in the transverse plane. And also the distance between the extrapolated track and the track segments in muon chamber, $\Delta x$ is required to be less than 3,5 and $6 \mathrm{~cm}$ for CMU, CMP and CMX respectively. Finally we make the isolation ratio cut with $I<0.1$. COT tracks are required to have at least 3 axial and 3 stereo layers with 7 hists each for both electron and muon identifications.

\subsection{Jet Identification and Corrections}

The jet reconstruction in this paper employs a cone cluster algorithm with cone radius $\Delta R \equiv$ $\sqrt{\Delta \eta^{2}+\Delta \phi^{2}}=0.4$ [36]. We measure the transverse energy $E_{T}=E \sin \theta$, where $\theta$ is the polar angle of the centroid of the clusters towers and calculated using the measured $z$ position of the event vertex. Total energy $E$ is the sum of the energy deposited in calorimeter towers within a cone. Jets are identified as isolated cluster and which contain significant hadronic energy where its measurements have a resolution of $\sigma / E_{T}=50 \% / \sqrt{E_{T}(G e V)} \oplus 3 \%$ [27].

The raw measurements are corrected for non-instrumented regions, non-linear response of the calorimeter, multiple interactions at high luminosity and other effects before they are used to reconstruct the top quark mass. In order to obtain estimates of the momenta of the daughter partons in the $t \bar{t}$ decay, the corrections occur in two stages.

- A set of "flavor-independent" or "generic" corrections

- A second set of corrections; the transfer functions. (specific to $t \bar{t}$ events) 
We call a first set of corrections "Flavor-independent" or "generic" because they are intended to be independent of the particular process under consideration. The details of $t \bar{t}$ specific corrections i.e. the transfer functions are discussed in Section 6.3 later. In this section, we focus on a set of generic corrections. Generic corrections are found by a program of study which examines the transverse energy balance in the di-jet and the $\gamma+$ jet samples. In CDF, it consists of seven steps of corrections to account for various effects. We briefly list the jet energy correction below:

- Level 1 : The $\eta$ Dependence in Calorimeter Response: Correction to make the calorimeter response uniform in $\eta$ by using the di-jet balancing procedure and is tested by the $\gamma+$ jet samples in data and Monte Carlo simulation.

- Level 2 : Calorimeter Stability (Time dependence): Correction for the variation in phototube response due to aging effects, therefore the correction is applied only to the data sample.

- Level 3 : Raw Scale for Central Calorimeter Responses: Scaling from Run II to Run I energy scale by studying $\gamma$-jet balancing (jets in the central region). Because we still use Run I absolute correction (Level 5) for now.

- Level 4 : Multiple Interaction: It is possible to have more than one interaction per collision due to high-luminosity collisions at Tevatron. This correction takes into account them. It is derived from minimum bias data and is parameterized as a function of the number of vertices in the event.

- Level 5: Absolute Energy Scale: This correction accounts for the calorimeter non-linearity, energy loss in the non-instrumented regions of each calorimeter and fragmentation. (Using Run I correction factor)

- Level 6 : Underlying Event correction: Measured from minimum bias data by requiring events with one vertex.

- Level 7 Out Of Cone Energy: This correction allows us to correct the particle level jet energy to the parton level by taking into account energies from initial parton which are not included in jet clustering.

Each correction factor has each uncertainty described in Section 8 in more details. Up to correction level 4, the scale factor is different between the data and Monte Carlo simulation, but 
from level 5 to 7 , the scale factors are the same. Therefore, our event selection is done using jets corrected up to level 4. Figure 3.1 illustrates the correction (scale) factors for level 4 to 7 as a function of $p_{T}$ and $\eta$ of the jets. As one can see in figure 3.1, they have strong dependence of $p_{T}$ and $\eta$ of the jets. Up to level 4 correction, scale factor is about $5 \%$ on average which is relatively small compared to the ones at level 5 and 7. Although we have correction level up to 7 which is supposed to make a jet back to a parton level, those corrections are extracted based on mainly QCD process which is not necessarily $t \bar{t}$ events. For top mass measurement, therefore we use corrections up to level 5 and after that, we use the transfer functions described in section 6.3 to account for the different fragmentation of light quark and gluon jets from W boson daughter jets and $b$ jets for specifically $t \bar{t}$ events. For reference, figure 3.2 shows dijet balance as a function of $\eta$.
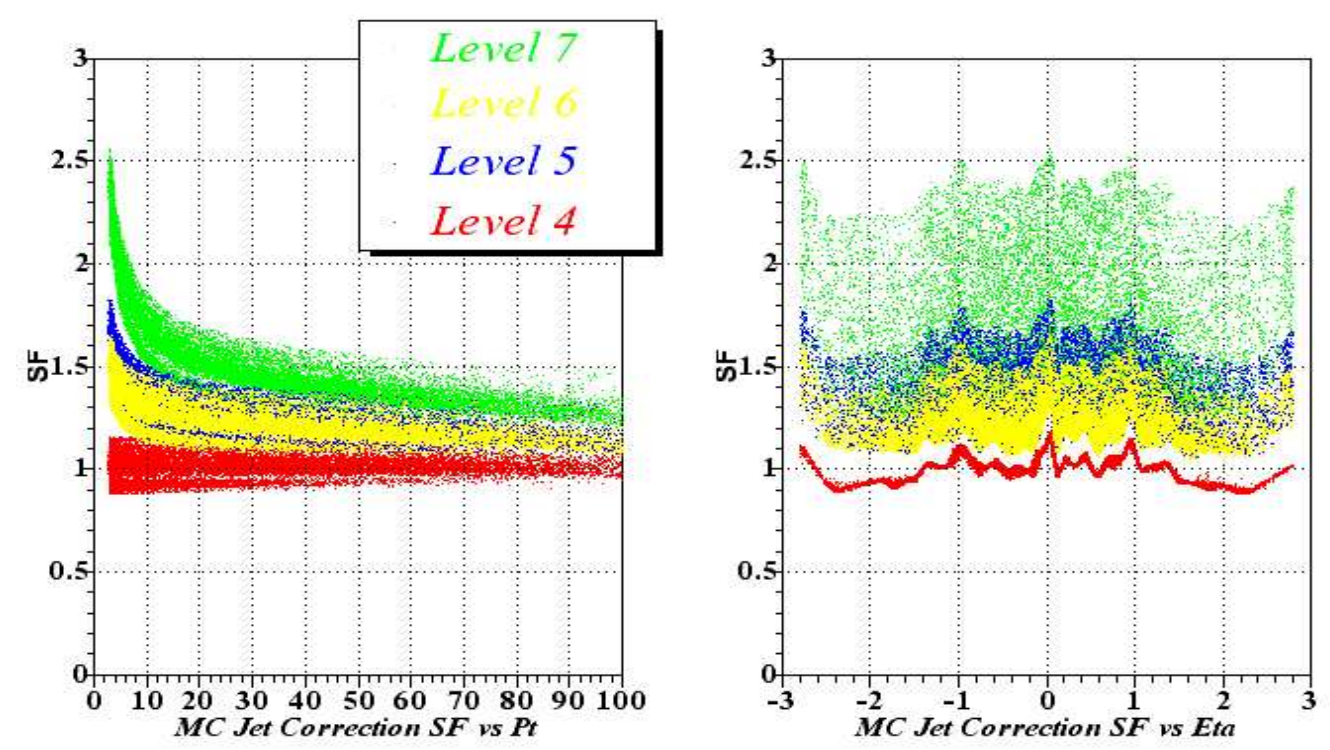

Figure 3.1: Generic correction scale factors for level 4 to 7 as a function of $p_{T}$ (left) and $\eta$ (right) of jets.

\section{3 $\quad b$ Jet Tagging using Secondary Vertex}

The identification of $b$ jets from top quark decay plays an important role in this analysis. Since most of the events coming from non- $t \bar{t}$ process passing all $\mathrm{W}+$ jets selection do not contain bottom or charm quarks in the final state, the requirement of presence of $b$ jets provides a 


\section{JetAna: dllot balance}

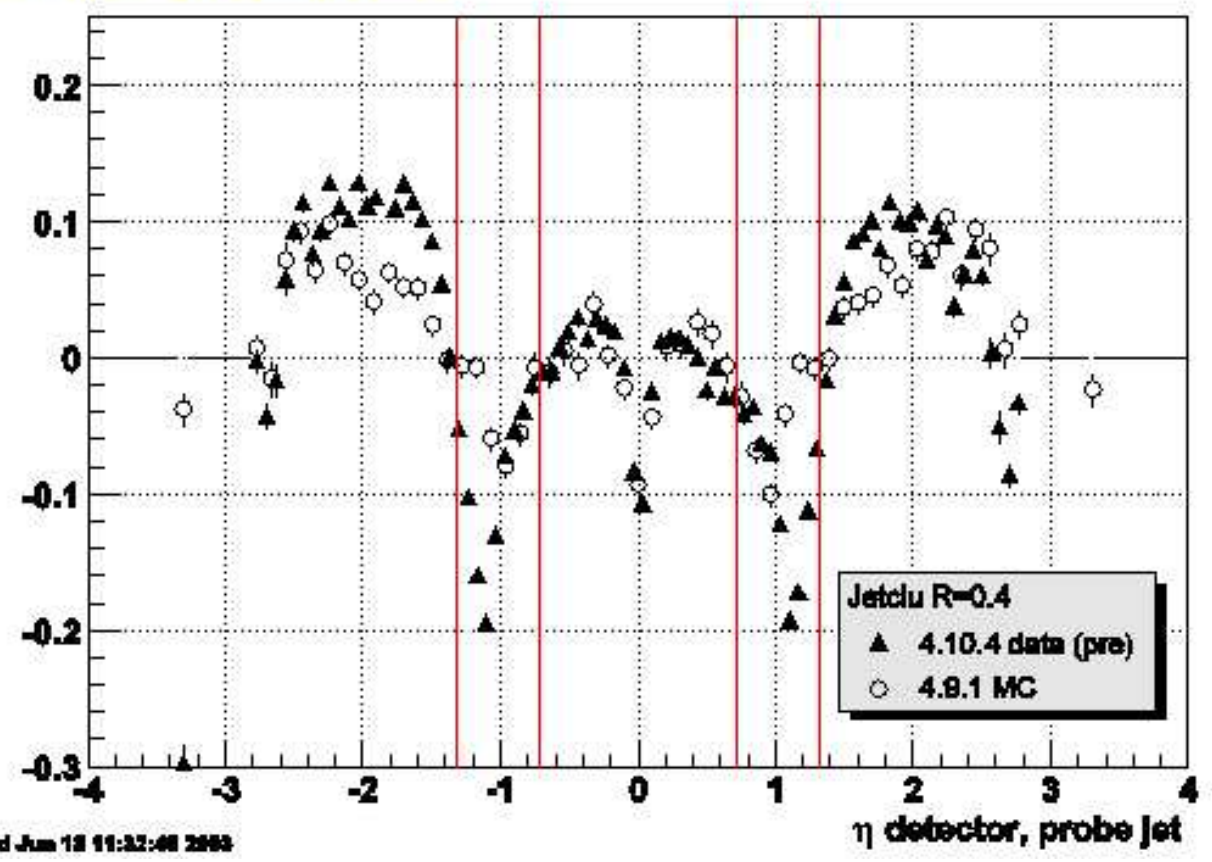

Figure 3.2: Dijet balance. The triangle is for data and open circle is for Monte Carlo simulation. There are three different regions of calorimeter called "Central" $(|\eta|<0.7)$, "Crack" or "Wall" $(0.7<|\eta|<1.3)$, and "Plug" $(|\eta|>1.3)$ 
significant reduction of the backgrounds.

The silicon vertex $b$ jet tag algorithm(SVX tagging) searches for the displacement of the secondary vertex due to $\mathrm{B}$ hadron decays to the primary vertex of the event within a jet $[37,38]$. It uses tracks which are within $\Delta R<0.4$ of the jet axis and have hits in the silicon detector. The algorithm performs as follows: (1) Select good tracks; which allows for two passes. In the first pass, at least three tracks with $p_{T}>0.5 \mathrm{GeV} / c$ and an impact parameter significance $\left|d_{0} / \sigma_{d_{0}}\right|>$ 2.5 are required where $\sigma_{d_{0}}$ includes the uncertainty on the track and beamline positions. At least one of the tracks must have $p_{T}>1 \mathrm{GeV} / c$. In the second pass, which requires tighter track requirements to have at least two tracks with $p_{T}>1 \mathrm{GeV} / c$ and $\left|d_{0} / \sigma_{d_{0}}\right|>3$, including at least one track with $p_{T}>1.5 \mathrm{GeV} / c$. (2) Reconstruct a secondary vertex using selected tracks. (3) Calculate the two-dimensional decay length of the secondary vertex $\left(L_{x y}\right)$ from the primary vertex. (4) Require to have $L_{x y} / \sigma_{L_{x y}}>3$, where $\sigma_{L_{x y}}$ is the estimated uncertainty on $L_{x y}$ which is typically $190 \mu \mathrm{m}$ to reduce the background from the false secondary vertices (mistags). Figure 3.3 shows a vertex display for one of the $t \bar{t}$ candidates, in which two jets are tagged as $b$ jets by finding secondary vertices. ( $L_{x y}=2.3 \mathrm{~mm}$ and $3 \mathrm{~mm}$ )

Based on simulation of the $b$ tagging algorithm, the $b$ tag requirement keeps $53 \%$ of the top quark events while removing more than $95 \%$ of the background events. The difference between the efficiency in the simulation and that in the data has been measured using a $b$-enriched dijet sample in which a non-isolated electron is found in a jet. We found data to Monte Carlo tagging efficiency scale factor of $0.82 \pm 0.06$ [38], which is used to extract the expected number of background from Monte Carlo. The error includes all systematic and statistical uncertainties.

\subsection{Missing Transverse Energy; $\boldsymbol{E}_{T}$}

The presence of neutrinos is inferred from transverse energy imbalance in the detector. The missing transverse energy is calculated as,

$$
E_{T}=-\left|\sum_{i} E_{T}^{i} \overrightarrow{n_{i}}\right|
$$

where, $E_{T}^{i}$ is the magnitude of the transverse energy contained in each calorimeter tower $i$ and $\overrightarrow{n_{i}}$ is the direction of the tower in the plane transverse to the beam direction. If isolated high$p_{T}$ muons are found in the event, the $\boldsymbol{E}_{T}$ is corrected by subtracting the muon energy in the calorimeter and adding the muon $p_{T}$ back to the vector sum. Further corrections for $\boldsymbol{E}_{T}$ to reconstruct the top mass related to jet energy corrections and the transfer functions can be 
shown in Section 6.3. An illustration of how to determine the missing energy can be shown in figure 3.3 which is a vertex display of an event. 


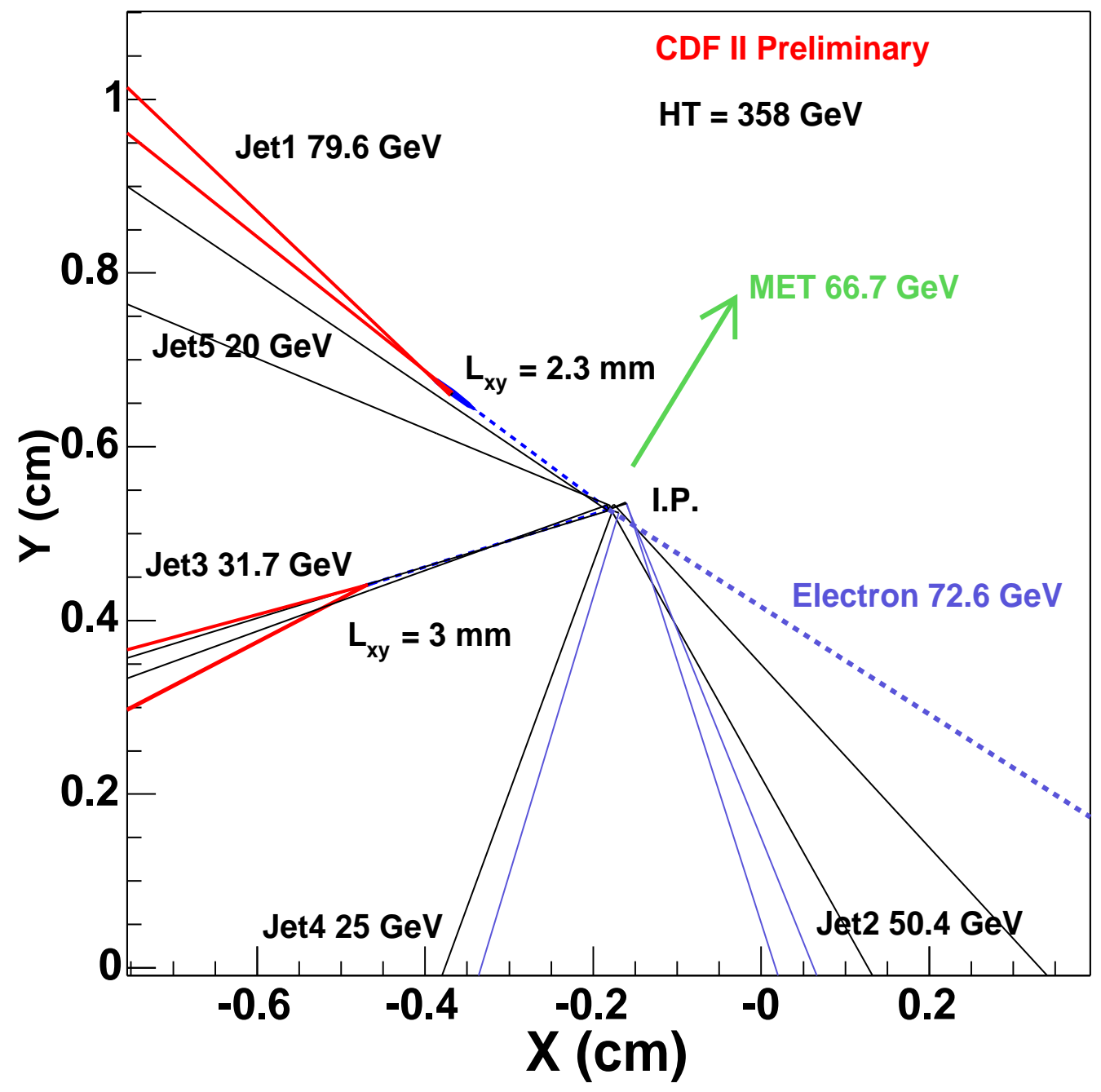

Figure 3.3: Vertex display of double tags in run 167551, event 7969376. I.P. stands for the interaction point (primary vertex). 


\section{Chapter 4}

\section{Data Samples and Event Selection}

\subsection{Data Samples}

The results reported here are based on the data which were recorded during the period March 2002 - August 2003, when the average instantaneous Tevatron luminosity was $\sim 2.0 \times 10^{31}$ $\mathrm{cm}^{-2} \mathrm{~s}^{-1}$, while the highest recorded $4.2 \times 10^{31} \mathrm{~cm}^{-2} \mathrm{~s}^{-1}$. The total integrated luminosity for this period is $197 \mathrm{pb}^{-1}$, of which $162 \mathrm{pb}^{-1}$ has good silicon tracking along with good electron and CMUP muon reconstruction, and $150 \mathrm{pb}^{-1}$ is for good CMX muons reconstruction.

As described previously, the CDF employs a three level trigger system for high momentum electrons and muons analysis for the primary data sets. The first two triggers (Level 1 and Level 2) are hardware online triggers and the third (Level 3) runs offline with a computer farm. For electron candidates, the level 1 trigger requires a track with $p_{T} \geq 8 \mathrm{GeV} / c$ matched to an EM calorimeter cell with $E_{T} \geq 8 \mathrm{GeV}$ and the ratio of the hadronic cell behind it to electromagnetic energy $\left(E_{\text {had }} / E_{\text {em }}\right)$ less than 0.125. After calorimeter clustering is made at level 2 , it requires a track with $p_{T} \geq 8 \mathrm{GeV} / c$ matched to an EM cluster with $E_{T} \geq 16 \mathrm{GeV}$. At level 3 which reconstructs electron cluster with calibration constants, $E_{T}>18 \mathrm{GeV}$ is required. For muon candidates, a track with $p_{T} \geq 8 \mathrm{GeV} / c$ matched to muon stubs in the muon chamber CMU, CMP, and CMX is required through level 1 to level 2. And then level 3 trigger requires a $p_{T} \geq$ $18 \mathrm{GeV} / c$ track. 


\subsection{Monte Carlo Programs}

The simulation of $t \bar{t}$ events relies mainly on the HERWIG v6.5 [39] and PYTHIA v6.2 [40] Monte Carlo program which employ leading order QCD matrix elements for the hard process , followed by parton showering to simulate gluon radiation and fragmentation with the CTEQ5L [41] parton distribution functions. For heavy flavor jets, the decay algorithm QQv9.1 [43] is used to provide proper modeling of B and C hadron decays. The ALPGEN program [44], which generates high multiplicity parton final states using exact leading-order matrix elements is used in our study of backgrounds in the b-tagged sample. The parton level events are then passed to HERWIG and QQ for fragmentation and B decay.

The CDF II detector simulation reproduces the response of the detector to particles produced in $p \bar{p}$ collisions. Tracking of particles through matter is performed with GEANT3 [45]. Charge deposition in the silicon detectors is calculated using a sample geometrical model based on the path length of the ionizing particle and an unrestricted Landau distribution. The drift model for the COT uses the GARFIELD package [24], with the default parameters tuned to match COT data. The calorimeter simulation uses the GFLASH [46] parameterization package interfaced with GEANT3. The GFLASH parameters are tuned to test beam data for electrons and high $p_{T}$ pions, and they are checked by comparing the calorimeter energy of isolated tracks in the collision data to their momenta as measured in the COT.

\subsection{Event Selection}

The final state of $t \bar{t}$ lepton+jets mode contains a high-momentum lepton, missing transverse energy that indicates the presence of neutrino from W leptonic decay, and four hadronic jets in which two jets are expected from $b$ quarks. To identify the candidates, we first select $\mathrm{W}+$ jets events which are mainly from non- $t \bar{t}$ process. To increase signal-to-background ratio, we require at least one $b$ jet tagged by SVX algorithm. We summarize the selection criteria below:

Only one isolated electron (muon) is required to have $E_{T} \geq 20 \mathrm{GeV}\left(p_{T} \geq 20 \mathrm{GeV} / c\right)$ and $|\eta| \leq 1.0$. Any event with two leptons satisfying the tight lepton identification (See Section 3.1) is removed. We also remove events where the second lepton is an electron in the plug calorimeter or a muon that fails the CMUP requirement, but has one CMU or CMP muon segment to remove top dilepton events $\left(t \bar{t} \rightarrow l^{+} \nu l^{-} \bar{\nu} b \bar{b} X\right)$. Missing Transverse Energy, $\boldsymbol{E}_{T}$ is required to

have greater than $20 \mathrm{GeV}$. Events with $\mathrm{Z}$ bosons candidates are removed by requiring that there is no second object which forms an invariant mass with the primary lepton within the window 
$[76,106] \mathrm{GeV} / c^{2}$. The second object is an opposite-signed isolated track with $p_{T}>10 \mathrm{GeV} / c$ for primary muons, and for primary electrons, it may be a track, an electromagnetic cluster, or a jet with $E_{T}>15 \mathrm{GeV}$ and $|\eta| \leq 2.0$ that has less than 3 tracks and high an electromagnetic energy fraction. The primary vertex of the event must be within $60 \mathrm{~cm}$ from the center of the CDF detector. The jets are clustered after removing towers containing electron clusters, and after correcting the tower $E_{T}$ for the location of the primary vertex $z$ coordinate. To minimize the contamination by initial and final state radiations, we select the events which have exactly and only four tight jets with $E_{T} \geq 15 \mathrm{GeV}$ and $|\eta| \leq 2.0$, i.e. unlike other methods [47], W + 3.5 jets (three tight jets and fourth jet with $E_{T} \geq 8 \mathrm{GeV}$ ) and $\mathrm{W}+\geq 5$ jets events are not used in this analysis. Finally, at least one SVX tagged $b$ jet is required. The number of observed events is shown in Table 4.1, together with the numbers in other jet multiplicity bin for reference. The above selection yields $22 b$ tag $\mathrm{W}+4$ jets events which are used to measure the top quark mass. As an example of the candidates, figures 4.1 and 4.2 show a vertex display and lego plot of energy deposited in calorimeters for double $b$ tagged event respectively. The overall selection efficiency for $t \bar{t}$ events in the lepton+jets channel with 4 jets is roughly $3 \%$ for the electron channel, $1 \%$ for muons in the CMP, and $0.5 \%$ for muons in the CMX.

\begin{tabular}{cccccc}
\hline \hline & W+1jet & W+2jets & W+3jets & W+4jets & W+ $\geq 5$ jets \\
\hline Electrons & 88 & 42 & 19 & 12 & 4 \\
Muons & 72 & 31 & 10 & 10 & 2 \\
\hline Total & 160 & 73 & 29 & 22 & 6 \\
\hline \hline
\end{tabular}

Table 4.1: The numbers of observed events with at least one $b$ tagged jet. 


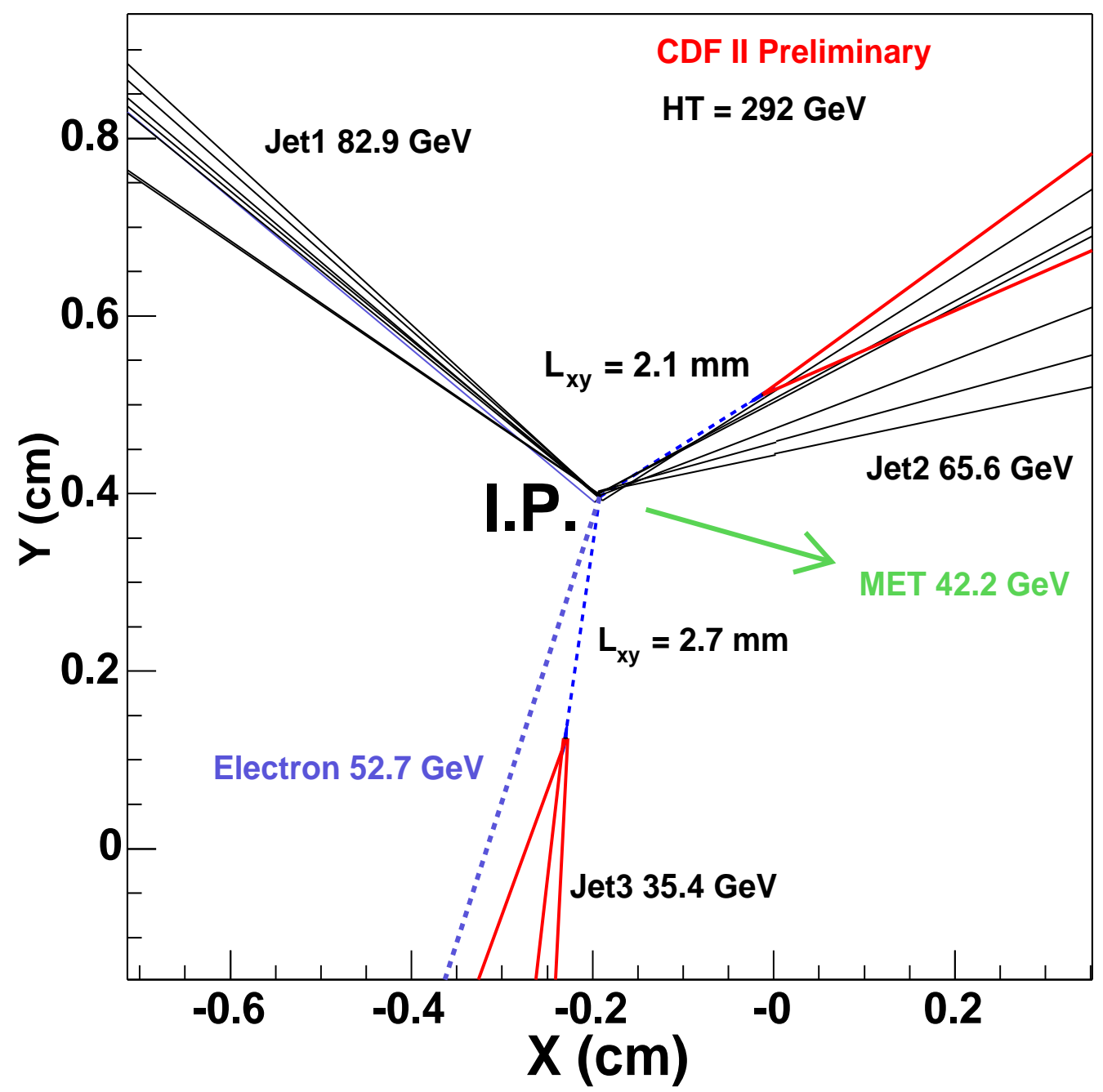

Figure 4.1: Vertex display of double tags in run 155145, event 132579 


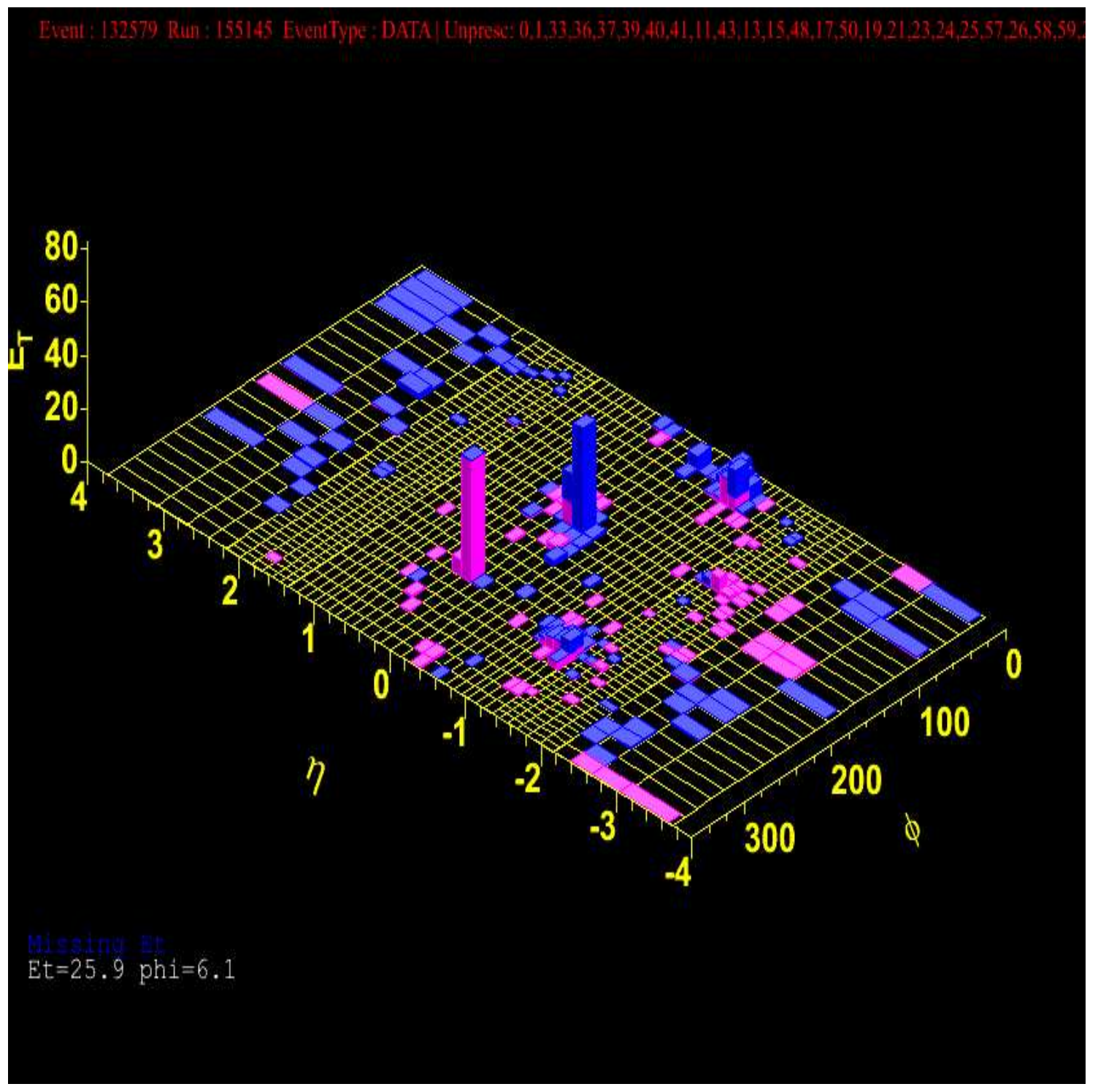

Figure 4.2: Lego plot of run 155145, event 132579, with an electron plus 3 jets 


\section{Chapter 5}

\section{Measurements of $t \bar{t}$ Production Cross Section}

\subsection{Overview}

In order to extract the top quark mass, a understanding and estimation of the background events are crucial. When we estimate the number of expected background or signal fraction in our sample, $t \bar{t}$ production cross section can be determined simultaneously. Therefore, the purpose of this chapter is to describe how the expected number of backgrounds $\left(N_{b k g}\right)$ or signal fraction $\left(f_{\text {sig }}\right)$ is determined to measure $t \bar{t}$ production cross section in CDF Run II. In our sample, following backgrounds are considered. (Note that $n p$ stands for $n$ light flavor quarks or gluons)

- Mistags : W plus light flavor quarks production with $b$ jet mis-tagging.

- $\mathrm{W} b \bar{b} 2 \mathrm{p}: \mathrm{W}$ plus heavy flavor production with $b \bar{b}$ quarks.

- $\mathrm{W} c \bar{c} 2 \mathrm{p}: \mathrm{W}$ plus heavy flavor production with $c \bar{c}$ quarks.

- Wc3p : W plus heavy flavor production with $c$ quark.

- Single top $s$ and $t$ channel: $s$-channel $q \bar{q}$ fusion and $t$-channel $W$-gluon fusion processes.

- WW/WZ/ZZ : Diboson production 
- $\mathrm{QCD}($ nonW $)$ : QCD production with fake leptons.

To estimate those contributions, two independent methods were already published in this channel (with SECVTX btagging). one is the counting method [38] and the other is the kinematic fitting method $[50,51]$. The results from these two methods are consistent each other within their uncertainty. In this section, only one of the two methods, a counting method is introduced. The number of background events to be used for this analysis is evaluated in the last section. Since the event selection for this mass measurement is slightly different from the samples used for cross section measurement in terms of the number of jets i.e. we use $W$ plus exactly four tight jets while cross section measurements use $W$ plus three or more tight jets, we need to re-evaluate the background from the one for cross section measurements.

\section{$5.2 t \bar{t}$ Production Cross Section}

\subsubsection{Counting Method}

In counting Method, the final cross section calculation, $\sigma_{t \bar{t}}=\left(N_{\mathrm{obs}}-N_{\mathrm{bkg}}\right) /\left(A_{t \bar{t}} \times \mathcal{L}\right)$, depends on the product $A_{t \bar{t}}$ of signal acceptance including selection efficiency, the expected number of non- $t \bar{t}$ background events $N_{\mathrm{bkg}}$, and the integrated luminosity $\mathcal{L}$. We need to estimate $A_{t \bar{t}}$ and $N_{\text {bkg }}$ by using Monte Carlo and data samples properly. Because the $t \bar{t}$ signal is expected to contribute significantly to the sample of events with $W+3$ jets or $W+\geq 4$ jets, an excess of observed events over the expected background with those jet multiplicities is assumed to be entirely due to $t \bar{t}$ production. The observed results for events with $W+1$ jet or $W+2$ jets, where the $t \bar{t}$ contribution is negligible, serve as a check of the background prediction. The non-t $\bar{t}$ events in the $W+$ jets sample are listed above. The estimation of each of these backgrounds is described in turn. The event selections used for this measurement are basically the same as an criteria for our top quark mass measurement, but for an optimization, we apply an additional cut using the variable $H_{T}$. $H_{T}$ is defined as the scalar sum of the transverse energy of all kinematic objects in the event, including all jets with $E_{T}>8 \mathrm{GeV}$ and $|\eta|<2.5$ :

$$
H_{T}=\sum_{\text {alljets }} E_{T}+\not E_{T}+E_{T}^{\text {electron }}\left(p_{T}^{\text {muon }}\right)
$$

A cut requiring $H_{T}>200 \mathrm{GeV}$ is found to be optimal: such a cut is expected to keep $96 \%$ of the signal, and reject $39 \%$ of the backgrounds; this would improve the signal over background ratio by $50 \%$ (from 2 to 3 ), and the total significance on the $t \bar{t}$ cross section measurement by 
$7 \%$. Systematic uncertainties due specially to the $H_{T}$ cut are small enough to be neglected in the optimization process. However, for the mass measurement, we do not apply this cut because $H_{T}$ has a strong correlation to the reconstructive top quark masses, it is better to avoid any biases that possibly happen by this requirement. We could however estimate the background contributions without $H_{T}$ cut as well.

In the following, we refer to "pretag sample" as the set of events passing all selection requirements, except for $b$-tagging.

\section{$t \bar{t}$ Signal Acceptance : $A_{t \bar{t}}$}

The acceptance is defined as the fraction of produced $t \bar{t}$ events that are actually observed. It includes trigger efficiency, reconstruction efficiency, as well as the efficiency of the kinematic cuts, and of the $b$-tagging algorithm. The branching ratio of the various decay modes of the $t \bar{t}$ pair must be taken into account as well. We split the acceptance into different contributions as follows:

$$
A_{t \bar{t}}=\epsilon_{t r i g} \cdot \epsilon_{z_{0}} \cdot \epsilon_{\text {veto }} \cdot \epsilon_{t \bar{t}}^{M C} \cdot k_{l e p t o n-i d} \cdot \epsilon_{t a g-e v e n t}
$$

where $\epsilon_{z_{0}}$ is the efficiency of the $\left|z_{0}\right| \leq 60 \mathrm{~cm}$ cut, $\epsilon_{t \bar{t}}^{\mathrm{MC}}$ is the fraction of Monte Carlo $t \bar{t}$ events which pass all the selection cuts (except for $b$-tagging), $\epsilon_{\mathrm{veto}}$ is the combined efficiency of the various vetoes (conversion removal, cosmic removal, dilepton and $Z^{0}$ rejections), $\epsilon_{\text {trig }}$ is the trigger efficiency for identifying high $p_{T}$ leptons, and $\epsilon_{\text {tag-event }}$ is the efficiency to tag at least one jet in a $t \bar{t}$ event. $k_{\text {lepton-id }}$ is a factor that corrects for the lepton identification efficiency difference between data and Monte Carlo.

The efficiency of the $z_{0}$ cut is measured from data and found to be $\epsilon_{z_{0}}=0.951 \pm 0.005$. The trigger efficiency $\epsilon_{\text {trig }}$ is different for each type of lepton trigger: $\epsilon_{\text {trig }}^{C E M}=0.9656 \pm 0.0006$, $\epsilon_{\text {trig }}^{C M U P}=0.887 \pm 0.007$, and $\epsilon_{\text {trig }}^{C M X}=0.954 \pm 0.006$. The factor $k_{\text {lepton-id }}$ is evaluated by comparing a data sample of $Z+$ jets events with a PYTHIA $Z$ sample, and found to be 1.00 for electrons, 0.95 for CMUP muons, and 0.99 for CMX muons; because there are few $Z+$ jets events at high jet multiplicity, we assign a $5 \%$ relative systematic uncertainty on $k_{\text {lepton-id }}$.

The efficiency $\epsilon_{t \bar{t}}^{\mathrm{MC}}$ is evaluated using a sample of PYTHIA $t \bar{t}$ Monte Carlo events with top quark mass $m_{t}=175 \mathrm{GeV} / c^{2}$. Table 5.1 summarizes the event selection acceptance for each type of lepton. The $b$-tagging efficiency is measured from the same Monte Carlo sample, and takes into account the $b$-tagging scale factor by randomly keeping only $82 \%$ of all the tags, and discarding the others. We find the efficiency for tagging at least one jet in a $t \bar{t}$ event (after 


\begin{tabular}{lcccc}
\hline \hline & CEM & CMUP & CMX & Total \\
\hline Sample (total) & 344,264 & 344,264 & 344,264 & 344,264 \\
\# Events w/o b-tag & 15,893 & 9791 & 3617 & 29301 \\
Acc. w/o b-tag (\%) & $4.09 \pm 0.03 \pm 0.36$ & $2.13 \pm 0.02 \pm 0.19$ & $0.959 \pm 0.016 \pm 0.085$ & $7.18 \pm 0.04 \pm 0.49$ \\
\hline \# Tagged Events & 8490 & 5202 & 1965 & 15657 \\
Tag Efficiency (\%) & $53.4 \pm 0.4 \pm 3.2$ & $53.1 \pm 0.5 \pm 3.2$ & $54.3 \pm 0.8 \pm 3.3$ & $53.4 \pm 0.3 \pm 3.2$ \\
Acc. with b-tag (\%) & $2.19 \pm 0.02 \pm 0.23$ & $1.14 \pm 0.01 \pm 0.12$ & $0.512 \pm 0.009 \pm 0.054$ & $3.84 \pm 0.03 \pm 0.40$ \\
Integ. Lumi. $\left(\mathrm{pb}^{-1}\right)$ & $162 \pm 10$ & $162 \pm 10$ & $150 \pm 9$ & \\
\hline \hline
\end{tabular}

Table 5.1: Summary table of the $t \bar{t}$ acceptance, for a top quark mass of $175 \mathrm{GeV} / c^{2}$.

all other cuts have been applied, including $H_{T}>200 \mathrm{GeV}$ ) to be $53.4 \pm 0.3$ (stat.) \pm 3.2 (syst.)\%. The systematic uncertainty comes from the measurement of the $b$-tagging scale factor. The overall acceptance is $3.84 \pm 0.03$ (stat.) \pm 0.40 (syst.)\%, including all systematic effects. Table 5.2 summarizes the dominant sources of uncertainty for the acceptance.

\section{Non-W QCD Background}

The non- $W / Z$ background is estimated directly from the data separately for the electrons and muons. These events include fake lepton and missing energy as well as semileptonic B decays. Real W events have the isolated primary lepton from a W decay and large $\boldsymbol{E}_{T}$ due to the neutrino, while non-W events tend to be opposite type. To extrapolate the number of non-W events in the sample, we use 2-dimensional plot of $\boldsymbol{E}_{T}$ and lepton isolation by defining four regions as,

- A: isolation $>0.2$ and $\boldsymbol{E}_{T}<15 \mathrm{GeV}$,

- B: isolation $<0.1$ and $\boldsymbol{E}_{T}<15 \mathrm{GeV}$,

- C: isolation $>0.2$ and $\boldsymbol{E}_{T}>20 \mathrm{GeV}$,

- D: isolation $<0.1$ and $\boldsymbol{F}_{T}>20 \mathrm{GeV}$.

A typical plot of $\boldsymbol{E}_{T}$ vs isolation can be shown in figure 5.1. Clearly, region D corresponds to W signal events. For the non-W QCD background, these two variables are uncorrelated, therefore the ratio of fakes at low and high isolation in the low $\boldsymbol{E}_{T}\left(N_{B} / N_{A}\right)$ is the same as the one in 


\begin{tabular}{lc}
\hline \hline Quantity & Relative error (\%) \\
\hline$\epsilon_{z_{0}}$ & 0.5 \\
Tracking Efficiency & 0.4 \\
Energy Scale & 4.9 \\
PDF & 2.0 \\
ISR/FSR & 2.6 \\
MC modeling & 1.4 \\
Lepton ID & 5.0 \\
$b$-tagging & 6.0 \\
\hline \hline
\end{tabular}

Table 5.2: Relative systematic uncertainties on the signal acceptance which are common to all lepton types.

the high $\boldsymbol{E}_{T}\left(N_{D} / N_{C}\right)$. The number of QCD contamination in region $\mathrm{D}$ is then calculated as, $N_{D}(\mathrm{QCD})=\frac{N_{B} \times N_{C}}{N_{A}}$.

Since $\mathrm{W}+$ Heavy Flavor backgrounds $(W b \bar{b}, W c \bar{c}$, and $W c)$ and mistags are estimated by normalizing to the number of the pretag(before $b$ tag) events, the contributions of non-W background for both the pretag and the tagged sample have to be measured even though we use only the tagged sample estimate in this mass measurement directly. To evaluate the expected number of events in the tagged sample, we use two methods: One is to estimate $N_{D}$ directly from the tagged sample, however it is limited by the low statistics, hence we lower the isolation boundary from 0.2 to 0.1 for region A and C. A second method is to scale the estimate in the pretag sample by tagging rate for QCD events. The events in region B with 2 or more jets is used to obtain a reliable tag rate [38]. The final estimate is obtained from the weighted average of two methods. These two estimates were found to be consistent with each other.

\section{Mistags}

SVX tags in a jet without a heavy flavor quark mainly due to symmetric track resolution is called a "mistag". The mistag rate per jet is measured from a large amount of inclusive jet data sample without relying on the detector simulation, by parameterizing as a function of the number of tracks, the raw jet $E_{T}$, the $\eta$ and $\phi$ of the jet, and the sum of the $E_{T}$ for all jets in the event with $E_{T}>10 \mathrm{GeV}$ and $|\eta|<2.4$. To estimate the size of the mistag background, each jet is weighted with its mistag rate in the pretag sample and then the sum of the weights over all jets in the 


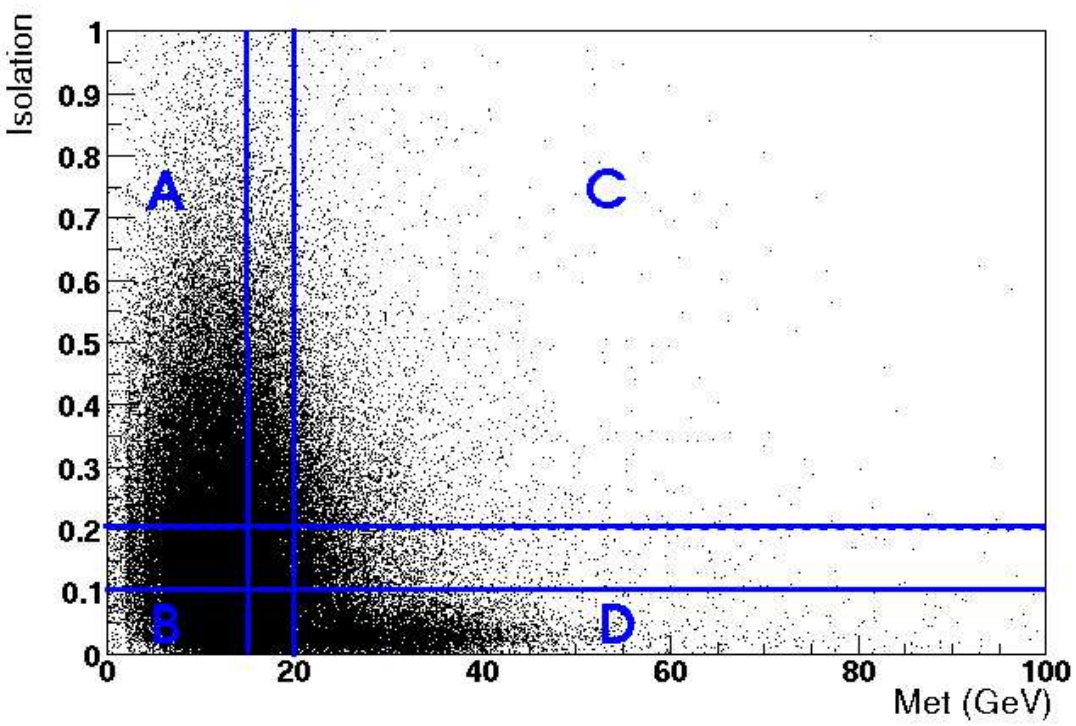

Figure 5.1: Met vs lepton isolation plot. Region D corresponds to our signal samples.

\begin{tabular}{|c|c|c|c|c|c|c|}
\hline \multirow[b]{2}{*}{ Jet multiplicity } & \multirow[b]{2}{*}{1 jet } & \multirow[b]{2}{*}{2 jets } & \multicolumn{2}{|c|}{$H_{T}>0$} & \multicolumn{2}{|c|}{$H_{T}>200 \mathrm{GeV}$} \\
\hline & & & 3 jets & $\geq 4$ jets & 3 jets & $\geq 4$ jets \\
\hline \multicolumn{7}{|c|}{ Electrons } \\
\hline Pretag non- $W$ QCD Fraction & $0.14 \pm 0.04$ & $0.17 \pm 0.04$ & \multicolumn{4}{|c|}{$0.20 \pm 0.05$} \\
\hline Tag Rate Method & $16.3 \pm 4.7$ & $7.4 \pm 2.2$ & \multicolumn{2}{|c|}{$3.2 \pm 1.0$} & \multicolumn{2}{|c|}{$2.1 \pm 0.6$} \\
\hline Tag Sample Method & $21.8 \pm 3.8$ & $10.0 \pm 2.2$ & \multicolumn{2}{|c|}{$4.9 \pm 1.3$} & \multicolumn{2}{|c|}{$2.6 \pm 0.8$} \\
\hline Combined Tag Estimate & $19.6 \pm 3.0$ & $8.7 \pm 1.6$ & $2.7 \pm 0.6$ & $1.1 \pm 0.2$ & $1.3 \pm 0.3$ & $1.0 \pm 0.3$ \\
\hline \multicolumn{7}{|c|}{ Muons } \\
\hline Pretag non- $W$ QCD Fraction & $0.034 \pm 0.010$ & $0.043 \pm 0.011$ & \multicolumn{4}{|c|}{$0.075 \pm 0.023$} \\
\hline Tag Rate Method & $4.0 \pm 1.3$ & $1.2 \pm 0.6$ & \multicolumn{2}{|c|}{$0.7 \pm 0.3$} & \multicolumn{2}{|c|}{$0.5 \pm 0.1$} \\
\hline Tag Sample Method & $4.8 \pm 1.1$ & $1.8 \pm 0.5$ & \multicolumn{2}{|c|}{$1.3 \pm 0.4$} & \multicolumn{2}{|c|}{$1.0 \pm 0.3$} \\
\hline Combined Tag Estimate & $4.5 \pm 0.8$ & $1.5 \pm 0.4$ & $0.7 \pm 0.2$ & $0.2 \pm 0.1$ & $0.3 \pm 0.1$ & $0.3 \pm 0.1$ \\
\hline Electron+Muon & $24.3 \pm 3.5$ & $10.5 \pm 1.9$ & $3.4 \pm 0.7$ & $1.3 \pm 0.3$ & $1.6 \pm 0.4$ & $1.2 \pm 0.4$ \\
\hline
\end{tabular}

Table 5.3: non- $W$ QCD background estimate. Results from the tag rate method and the tag sample method are the number of events expected in the b-tagged lepton + jets sample. 
sample is counted after scaling down by the fraction of pretag events which are due to non-W background to avoid a double counting between these two background estimates. It is a good estimate that the number of mistagged jets is used as the number of mistagged events, because the mistag rate per jet is sufficiently low, typically $1 \%$. This method is tested by comparing the negative SVX tags observed and predicted for the pretag sample as a function of the jet $E_{T}$. We have observed a fair agreement in the shape and normalization of the prediction [38].

\section{W + Heavy Flavor Backgrounds}

The production of $\mathrm{W}$ bosons accompanied by QCD production of heavy flavor quarks in the processes $W b \bar{b}, W c \bar{c}$, and $W c$ produces very similar signature to $t \bar{t}$ events in the final state and a significant part of the background for the tagged sample. In order to evaluate this background, we use the Monte Carlo models ALPGEN+HERWIG [44,39] to estimate the fraction of the inclusive $\mathrm{W}+$ jets events containing $\mathrm{W} b \bar{b}, \mathrm{~W} c \bar{c}$, and $\mathrm{W} c$ by matching final state partons to reconstructed jets and multiply the heavy flavor fractions by the pretag event count, after subtracting the non-W backgrounds. The heavy flavor fractions are measured to be approximately $2 \sim 3 \%$ for $W b \bar{b}$ and $W c \bar{c}$, and $6 \%$ for $W c$ events and were calibrated by comparing di-jet Monte Carlo events with that of data. Estimates of the tagged background are then obtained by multiplying the tagging efficiency of each background source. More details on all calculations can be found in $[38]$.

\section{Other Backgrounds}

The $W W, W Z$, and $Z Z$ background, $Z \rightarrow \tau \tau$, and electroweak single top of both $s$-channel $q \bar{q}$ fusion and $t$-channel $W$-gluon fusion processes are evaluated based on the predictions from Monte Carlo simulation by multiplying the acceptances for these processes as determined from the PYTHIA Monte Carlo program using their production cross sections [48, 49] and measured integrated luminosity. The Monte Carlo acceptance is corrected for the differences between the Monte Carlo and the data of the lepton identification and trigger efficiencies with their uncertainties. And also the $b$ tagging efficiency is scaled by the $\mathrm{MC} /$ data tagging scale factor which was described in Sec. 3.3. 


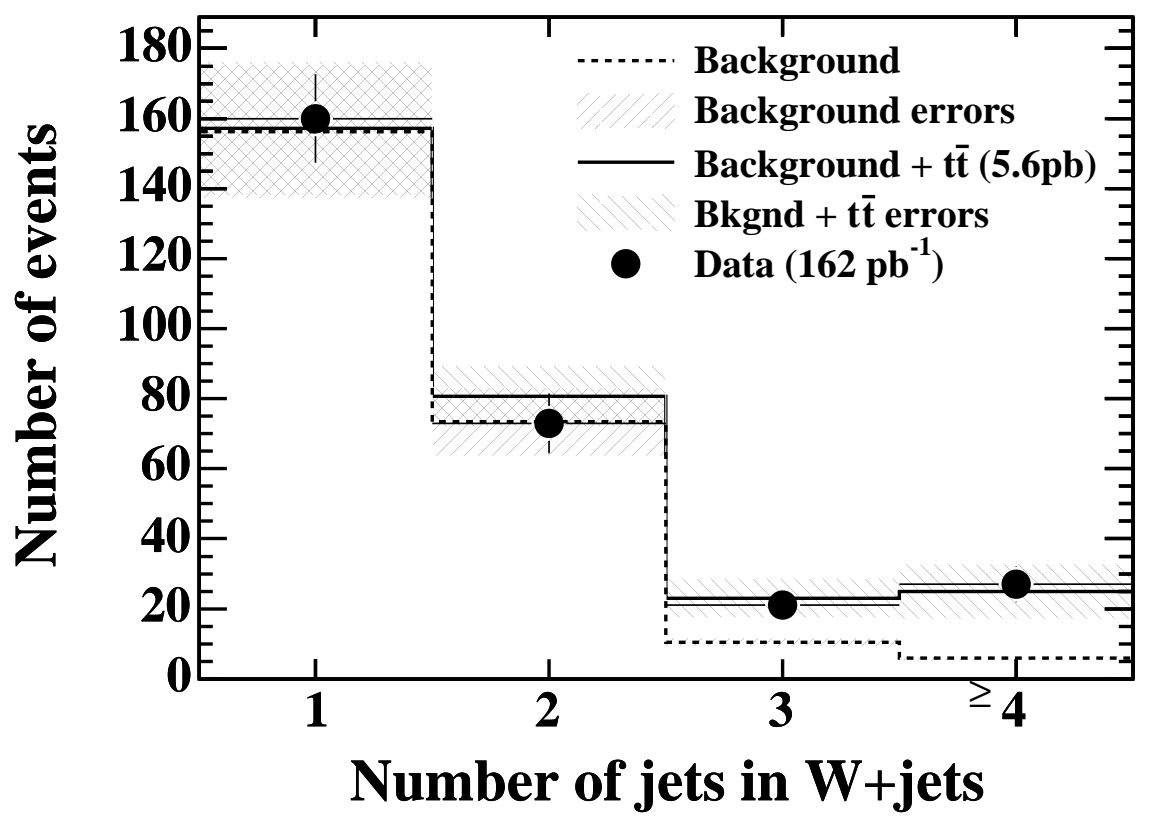

Figure 5.2: Background and $t \bar{t}$ signal expectation (based on measured $t \bar{t}$ cross section) as a function of jet multiplicity. Events with three or more jets are required to have $H_{T}>200 \mathrm{GeV}$.

\section{Summary}

Figure 5.2 shows that the contribution of the different backgrounds for each jet bin compared to the observed number of tagged events in the data. By looking at good agreement between the background and the data in the 1 and 2 jet bins, our calculation is found to be well validated. The excess of tags in the 3 and 4 jet bins is attributed to $t \bar{t}$ signal events.

The production cross section follows from the acceptance measurement and the background estimate:

$$
\sigma_{t \bar{t}}=\frac{N_{\mathrm{obs}}-N_{\mathrm{bkg}}}{\epsilon_{t \bar{t}} \times \mathcal{L}}
$$

where $N_{\text {obs }}$ and $N_{\text {bkg }}$ are the number of total observed and background events, respectively, in the $W+\geq 3$ jet bins (see Table 5.4); $\epsilon_{t \bar{t}}$ is the signal acceptance (see Table 5.1); and $\mathcal{L}$ is the integrated luminosity. Many of the predicted backgrounds are based on the number of pretag data events, but that number includes a significant contribution from $t \bar{t}$ events. After subtracting this contribution from the pretag sample, the dependent backgrounds are recalculated. The final background contributions for the single-tag selection are summarized in 


\begin{tabular}{ccc|cccc}
\hline \hline & & \multicolumn{2}{|c}{$H_{T}>0 \mathrm{GeV}$} & \multicolumn{2}{c}{$H_{T}>200 \mathrm{GeV}$} \\
Jet multiplicity & $W+1$ jet & $W+2$ jets & $W+3$ jets & $W+\geq 4$ jets & $W+3$ jets & $W+\geq 4$ jets \\
\hline Pretag & 15314 & 2448 & 387 & 107 & 179 & 91 \\
Mistags & $40.9 \pm 6.1$ & $17.0 \pm 2.4$ & $5.2 \pm 0.7$ & $2.6 \pm 0.4$ & $3.3 \pm 0.4$ & $2.3 \pm 0.3$ \\
$W b \bar{b}$ & $37.0 \pm 11.2$ & $22.5 \pm 6.5$ & $5.0 \pm 1.3$ & $1.6 \pm 0.5$ & $2.8 \pm 0.8$ & $1.4 \pm 0.4$ \\
$W c \bar{c}$ & $13.7 \pm 3.4$ & $8.0 \pm 2.2$ & $1.6 \pm 0.5$ & $0.6 \pm 0.2$ & $0.9 \pm 0.3$ & $0.5 \pm 0.2$ \\
$W c$ & $34.5 \pm 9.0$ & $7.7 \pm 2.0$ & $1.4 \pm 0.4$ & $0.3 \pm 0.1$ & $0.7 \pm 0.2$ & $0.3 \pm 0.1$ \\
non- $W$ QCD & $2.2 \pm 0.4$ & $2.5 \pm 0.4$ & $0.6 \pm 0.1$ & $0.1 \pm 0.0$ & $0.3 \pm 0.1$ & $0.1 \pm 0.0$ \\
single top & $2.6 \pm 3.5$ & $10.5 \pm 1.9$ & $3.4 \pm 0.7$ & $1.4 \pm 0.4$ & $1.7 \pm 0.4$ & $1.2 \pm 0.3$ \\
$Z+\mathrm{HF}$ & $1.1 \pm 0.3$ & $0.6 \pm 0.2$ & & $0.2 \pm 0.1$ & $0.8 \pm 0.1$ & $0.2 \pm 0.0$ \\
Total & $156.3 \pm 19.1$ & $73.4 \pm 9.8$ & $18.5 \pm 2.2$ & $6.9 \pm 0.9$ & $10.5 \pm 1.3$ & $6.0 \pm 0.8$ \\
Corrected Total & $156.3 \pm 19.1$ & $73.4 \pm 9.8$ & & $23.1 \pm 3.0$ & & $13.5 \pm 1.8$ \\
Data & 160 & 73 & 29 & 28 & 21 & 27 \\
\hline \hline
\end{tabular}

Table 5.4: Background summary for the single-tag selection. The total backgrounds are given before and after the correction for $t \bar{t}$ events in the pretag $W+$ jets sample.

Table 5.4 and represented in Figure 5.2.

The properties of the selected candidate events are consistent with the expectations for $t \bar{t}$ pair production and background contributions. Figures 5.3 shows the distribution of the event $H_{T}$. For the optimized selection with the $H_{T}$ requirement, and for a top quark mass $m_{\mathrm{t}}=175 \mathrm{GeV} / c^{2}$,

$$
\left.\sigma_{t \bar{t}}=5.6_{-1.1}^{+1.2} \text { (stat. }\right)_{-0.6}^{+0.9}(\text { syst.)pb. }
$$

without $H_{T}$ cut,

$$
\sigma_{t \bar{t}}=5.3_{-1.1}^{+1.3}(\text { stat. })_{-0.7}^{+1.2}(\text { syst. }) \text { pb. }
$$

The systematic uncertainty is due to uncertainties on the signal acceptance ( $10 \%$ relative), luminosity measurement (6\%), and background estimate (5\%). The acceptance, and therefore the measured cross section, changes with the top quark mass as shown in Table 5.5. 


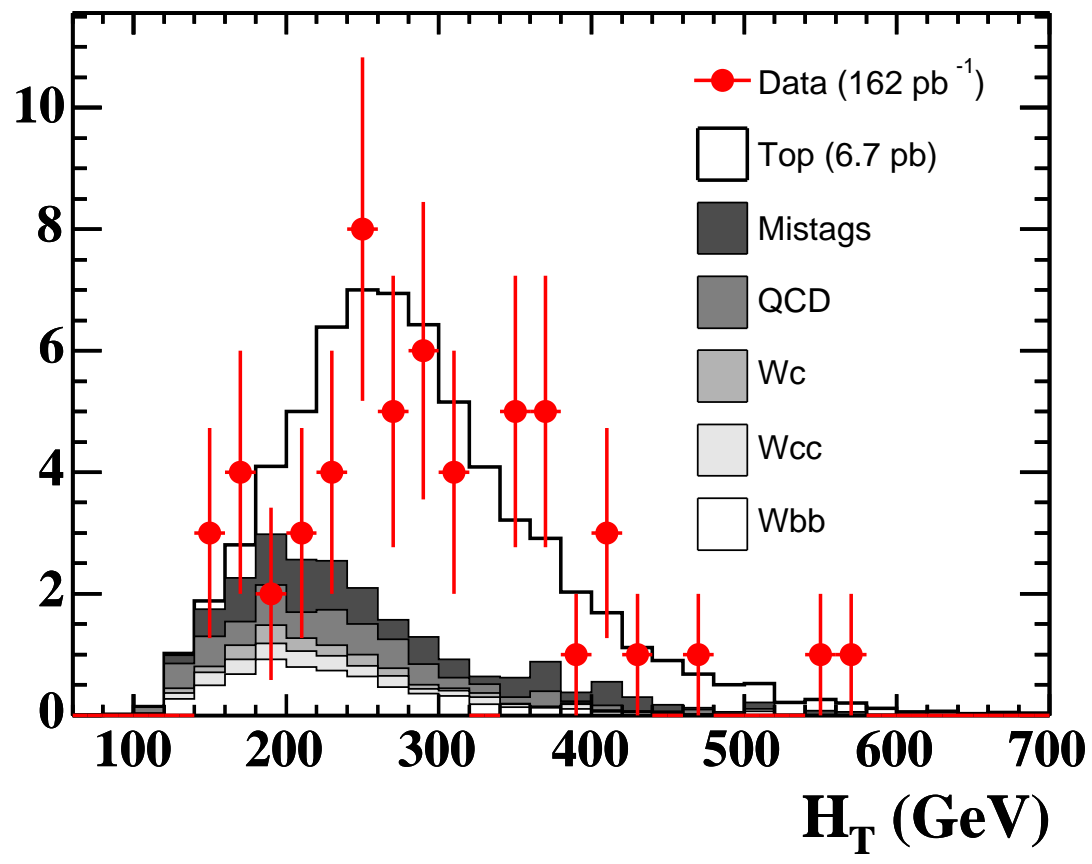

Figure 5.3: $H_{T}$ distribution of the 57 tagged events with three or more jets, compared to the expected background and $t \bar{t}$ signal (normalized to the theoretical cross-section of $6.7 \mathrm{pb}$ ).

\begin{tabular}{cccc}
\hline \hline mass $\left(\mathrm{GeV} / c^{2}\right)$ & 170 & 175 & 180 \\
\hline cross section $(\mathrm{pb})$ & $5.8 \pm 1.2$ & $5.6 \pm 1.2$ & $5.4 \pm 1.1$ \\
\hline \hline
\end{tabular}

Table 5.5: Measured cross section for different top quark mass assumptions. 


\subsection{Background Estimation used for this analysis}

As descrbied above, the measurements of $t \bar{t}$ cross section and its background estimations are done by using three or more jets bin in which three jets bin and four or more jets bin are estimated seperately. On the other hand, we use exactly four jets events for the mass measurement to reconstruct the top quark mass (3jets events can not form $t \bar{t}$ pair) and to minimize the contamination from initial/final state gluon radiations. Therefore we need to re-evaluate the background fraction in our candidate events. This has been done with very simple way: For each background, we calcuate the fraction between $\geq 4$ jets and $==4$ jets bin using Monte Carlo except for mistag and QCD backgrounds which are directly estimated from data. Typically the fraction of selection efficiency, $\epsilon(==4 \mathrm{jets}) / \epsilon$ ( $\geq 4 \mathrm{jets})$, is estimated to be about $80 \%$. By scaling the number of events in $\geq 4$ jets bin to those in $==4$ jets bin, we finally obtain all of the background contribution in our sample which are summarized in Table 5.6. We evaluate the total number of background events to be $4.2 \pm 0.7$. Also the expected number of signal events is 20.9 events assuming a cross section of $6.7 \mathrm{pb}$ [9]. These estimates are consistent with the number of observed events of 22 within the statistical fluctuation.

\begin{tabular}{c|c|c}
\hline \hline Model & Source & $\mathrm{W}+4$ jets bin \\
\hline $\mathrm{W} 4 \mathrm{p}(\mathrm{MC})$ & mistag & $1.2 \pm 0.37$ \\
$\mathrm{~W} b \bar{b}(\mathrm{MC})$ & $\mathrm{W} b \bar{b}$ & $0.7 \pm 0.29$ \\
$\mathrm{~W} c \bar{c}(\mathrm{MC})$ & $\mathrm{W} c \bar{c}$ & $0.3 \pm 0.12$ \\
$\mathrm{~W} c(\mathrm{MC})$ & $\mathrm{Wc}$ & $0.2 \pm 0.12$ \\
$\mathrm{WW}(\mathrm{MC})$ & $\mathrm{WW} / \mathrm{WZ} / \mathrm{ZZ}$ & $0.08 \pm 0.05$ \\
single top $(\mathrm{MC})$ & single top & $0.17 \pm 0.03$ \\
large iso(data) & $\mathrm{QCD}(\mathrm{nonW})$ & $1.6 \pm 0.38$ \\
\hline \multicolumn{2}{c}{ Total } & $4.2 \pm 0.71$ \\
\hline \multicolumn{2}{c}{ (t) expected $(6.7 p b)$} & 20.9 \\
\hline \hline
\end{tabular}

Table 5.6: The numbers of expected events for individual background sources in $\mathrm{W}+4 \mathrm{jets}$ sample. 


\section{Chapter 6}

\section{Measurement of Top Quark Mass}

\subsection{Analysis Overview}

The analysis proceeds as follows. A likelihood for the mass of top is calculated by the method called dynamical likelihood method (DLM) described in Section 6.2. In DLM, the likelihood is defined as the differential cross section per unit phase space of final partons in the elementary process. To infer parton momenta, we define the transfer function which correlates the observed quantities and the corresponding parton quantities: four quarks ( $q \bar{q}^{\prime}$ from $W, b$ and $\left.\bar{b}\right)$ and four jets. The transfer function is obtained from PYTHIA Monte Carlo $t \bar{t}$ signal samples. Section 6.3 describes the details and performance checks of the transfer function. There are 12 and 4 combinations of identifications of jets with partons, depending on whether 1 or 2 jets are btagged. Instead of selecting one good combination (e.g. giving maximum likelihood value) out of those, we sum likelihoods for all possible jet combinations in an event and such event likelihoods are multiplied each other to obtain the joint likelihood function of the data. After calculating the top quark mass based on the assumption that all events are signal $t \bar{t}$, effects of backgrounds are corrected by using a mapping function that provides a mass-dependent correction factor for the background contamination. The mapping function is extracted by pseudo-experiments mostly using Monte Carlo samples where background fraction is Poisson distributed. Finally, we extract our measured value of top quark mass using expected background fraction already estimated in Section 5.3. 


\subsection{Dynamical Likelihood Method}

The method we use in this paper is the Dynamical Likelihood Method(DLM) which was originally proposed in 1988[55] and developed[56][57], and details of the latest formulation are described in [58]. In DLM, we assume the standard model and use the differential cross section per unit phase space to infer the parton kinematics and the mass of the top quark. This section describes the definition of the likelihood and the procedure of the reconstruction.

\subsubsection{Differential cross section}

The parton process in a $p \bar{p}$ collision can be generally written as,

$$
a / p+b / \bar{p} \rightarrow C, \quad C \equiv \sum_{i=1}^{n} c_{i}
$$

where $a$ and $b$ are the initial partons, a quark or anti-quark or gluon, in the proton and antiproton, respectively, and $c_{1}, c_{2}, \ldots c_{n}$ are final state partons. States of partons are after initialand before final-state radiation. In the case of the $t \bar{t}$ lepton+jets channel, the initial parton set $(a, b)$ is $(q, \bar{q}),(\bar{q}, q)$ or $(g, g)$, and the final partons are $l, \nu, q, \overline{q^{\prime}}, b, \bar{b}$, where $l=e$ or $\mu$, Throughout this paper, a particle symbol $p$ represents its 4-momentum, and $\boldsymbol{p}$ its 3 -momentum. The final partons are assumed to have their pole masses so that the 3-momenta define their states unambiguously.

In general, the hadronic cross-section for $n$-body final state partons is given by

$$
d \sigma=d z_{a} d z_{b} d^{2} \boldsymbol{p}_{T} f_{a / p}\left(z_{a}\right) f_{b / \bar{p}}\left(z_{b}\right) f_{T}\left(p_{T}\right) d \hat{\sigma}(a+b \rightarrow C ; \boldsymbol{\alpha})
$$

where $d \hat{\sigma}$ is the parton level cross section[22],

$$
d \hat{\sigma}(a+b \rightarrow C ; \boldsymbol{\alpha})=\frac{(2 \pi)^{4}}{4 \sqrt{(a \cdot b)^{2}-m_{a}^{2} m_{b}^{2}}}|\mathcal{M}(a+b \rightarrow C ; \boldsymbol{\alpha})|^{2} d \Phi_{n}(a+b ; C) .
$$

In Eq. (6.2) symbol $\boldsymbol{\alpha}$ represents a set of dynamical constants to be measured, e.g. masses, decay widths and coupling constant ratios. In this analysis, $\boldsymbol{\alpha}$ is simply the top quark mass $M_{\text {top }}$. Variables $z_{a}$ and $z_{b}$ are the energy fractions of $a$ and $b$ in hadrons $p$ and $\bar{p}$ respectively, and $\boldsymbol{p}_{T}$ is the total momentum of the initial and final systems in the plane transverse to the beam axis. Functions $f_{a / p}\left(z_{a}\right)$ and $f_{b / \bar{p}}\left(z_{b}\right)$ denote the Parton Distribution Functions(PDFs), while $f_{T}$ is the probability function for the total transverse momentum of the system acquired by initial state radiation. In Eq. (6.3), $\mathcal{M}$ is the matrix element of the process which is being 
studied (in our case, $t \bar{t}$ production and decay), and $d \Phi_{n}$ is the Lorentz invariant phase space element,

$$
d \Phi_{n}=\delta^{4}(a+b-C) d \Phi_{n}^{(f)}
$$

where

$$
d \Phi_{n}^{(f)}=\prod_{i=1}^{n} \frac{d^{3} \boldsymbol{c}_{i}}{(2 \pi)^{3} 2 E_{i}} .
$$

We use Eqs.(6.2) and (6.3) to formulate the posterior probability, i.e. the parton level likelihood. The basic postulate is that final partons occupy an $n$-dimensional unit phase space in the neighborhood of $\boldsymbol{c}\left(c_{1}, \cdots, c_{n}\right)$. When momentum set $\boldsymbol{c}$ is given, the total probability for this final state to occur is obtained by integrating Eq. (6.2) with initial state variables, $z_{a}, z_{b}$ and $\boldsymbol{p}_{T}$, as

$$
\frac{d \sigma}{d \Phi_{n}^{(f)}}=I(a, b)|\mathcal{M}(a+b \rightarrow C ; \boldsymbol{\alpha})|^{2}
$$

where

$$
I(a, b)=\frac{(2 \pi)^{4}}{4|\boldsymbol{p} \| \overline{\boldsymbol{p}}| \sqrt{(a \cdot b)^{2}-m_{a}^{2} m_{b}^{2}}} f_{a / p}\left(z_{a}\right) f_{b / \bar{p}}\left(z_{b}\right) f_{T}\left(p_{T}\right)
$$

is the integration factor for the initial state. Because of the $\delta$-function in Eq. (6.3), the initial parton momenta $a$ and $b$ are uniquely defined by that of $C$.

When $\boldsymbol{c}$ is given, the parton level likelihood $L_{1}^{p}$ for $\boldsymbol{\alpha}$ is defined by

$$
L_{1}^{p}(\boldsymbol{\alpha} \mid \boldsymbol{c})=l_{0} \frac{d \sigma}{d \Phi_{n}^{(f)}}
$$

where $l_{0}$ is the expectation(average) value of the integrated luminosity to produce a single event. If a data set has a total a total number of $N_{e v}$ events with the integrated luminosity of $L_{i n t}, l_{0}$ is defined as

$$
\frac{L_{i n t}}{N_{e v}} \rightarrow l_{0}=\frac{1}{\sigma_{T}\left(\boldsymbol{\alpha}_{0}\right)} \quad\left(N_{e v} \rightarrow \infty\right)
$$

where $\sigma_{T}\left(\boldsymbol{\alpha}_{0}\right)$ is the total cross section for the true value of $\boldsymbol{\alpha}, \boldsymbol{\alpha}_{0}$.

\subsubsection{Transfer functions for observables}

Final partons are not directly observed; they undergo hadronization and are observed by detectors with finite resolution, and quarks and gluons are reconstructed as jets. General jet energy corrections are calibrated by QCD jets, so we need additional corrections for $b$ jets and W daughter jets in $t \bar{t}$ process. To describe the correlation between the final parton state and observed quantities (observables), we introduce the transfer function $w\left(\boldsymbol{x}|\boldsymbol{y}| \mid M_{t o p}\right)$, where $\boldsymbol{y}$ represents 
a set of observables and $\boldsymbol{x}$ is a parton variable set that corresponds to $\boldsymbol{y}$. In the lepton+jets process, $\boldsymbol{y}$ consists of the momenta of the $e$ or $\mu$ and of the 4 jets, and the missing transverse momentum $\left(\boldsymbol{E}_{T}\right)$.

The differential probability for the parton variables $\boldsymbol{x}$ to be observed as $\boldsymbol{y}$ is defined by transfer function $w(\boldsymbol{y} \mid \boldsymbol{x})$ as

$$
d P(\boldsymbol{y})=w(\boldsymbol{y} \mid \boldsymbol{x}) d \boldsymbol{y}
$$

We assume $w(\boldsymbol{y} \mid \boldsymbol{x})$ can be factorized into the individual observable components,

$$
w(\boldsymbol{y} \mid \boldsymbol{x})=\prod_{i} w\left(y_{i} \mid x_{i}\right)
$$

and require conservation of the probability,

$$
\int w\left(y_{i} \mid x_{i}\right) d y_{i}=1
$$

The transfer functions are obtained by using a $t \bar{t}$ Monte Carlo sample.

\subsubsection{Resonances}

When process (6.1) includes resonance $r$, i.e. the internal line of the Feynman graph, as

$$
\begin{gathered}
a / p+b / \bar{p} \rightarrow r+c_{j+1}+\cdots+c_{n} \\
r \rightarrow c_{1}+\cdots+c_{j}
\end{gathered}
$$

we assume the matrix element factorizes as

$$
|\mathcal{M}(a+b \rightarrow C ; \boldsymbol{\alpha})|^{2}=\left|\mathcal{M}_{\text {prod }}\right|^{2} \mathcal{P}\left(s_{r}\right)\left|\mathcal{M}_{\text {dec }}\right|^{2}
$$

where $\mathcal{M}_{\text {prod }}$ and $\mathcal{M}_{d e c}$ are matrix elements for process (6.13) and (6.14) respectively, and $s_{r}$ is the virtual mass of $r$, which satisfies

$$
s_{r}=\left(\sum_{i=1}^{j} c_{i}\right)^{2} .
$$

$\mathcal{P}\left(s_{r}\right)$ is the propagator factor,

$$
|\mathcal{P}|^{2} \equiv \Pi\left(s_{r}\right)=N \frac{M_{r} \Gamma_{r}}{\left(s_{r}-M_{r}^{2}\right)^{2}+M_{r}^{2} \Gamma_{r}^{2}},
$$

which satisfies the conservation of probability[40],

$$
\int_{0}^{\infty} \Pi(s) d s=1
$$

with $\mathrm{N} \approx \frac{1}{\pi}$. In $t \bar{t}$ production, $t, \bar{t}, W^{+}$and $W^{-}$are treated as resonances. 


\subsubsection{Reconstruction of parton kinematics}

The reconstruction of parton kinematics $c$ can be made by selecting $3 n$ components of variables out of the observable set $\boldsymbol{y}$ and a set of invariant masses of resonance $\boldsymbol{s}_{r}$. The condition for the reconstruction is observed quantity $\boldsymbol{y}$. We make statistical inference for possible parton kinematics by random generations of $\boldsymbol{c}$ and/or $\boldsymbol{s}_{r}$. We call a single set of parton kinematics a path. For many paths we generate, a phase space consideration is necessary.

The recursion formula for process (6.13) followed by (6.14) is given by

$$
\begin{array}{r}
d \Phi_{n}\left(a+b: c_{1}, \cdots, c_{n}\right)=d \Phi_{j}\left(r: c_{1}, \cdots, c_{j}\right)(2 \pi)^{3} 2 \delta\left(s_{r}-\left(\sum_{i=1}^{j} c_{i}\right)^{2}\right) d s_{r} \\
\times d \Phi_{n-j+1}\left(a+b: r, c_{j+1}, \cdots, c_{n}\right) .
\end{array}
$$

If all final partons are observed, $\boldsymbol{c}$ can be inferred with $w(\boldsymbol{y} \mid \boldsymbol{x})$, and the differential cross section for a single path is given by

$$
\frac{d \sigma}{d \Phi_{n}^{(*)}}=\left(\frac{d \sigma}{d \Phi_{n}^{(f)}}\right)_{\boldsymbol{c}}
$$

If $s_{r}$ is used for the reconstruction, whether all daughters are observed or not, the phase space of one parton e.g. $c_{1}$ is determined from $\left(r, c_{2}, \cdots, c_{j}\right)$ by Eq. (6.19). In this case, the cross section form to be used is derived from Eq. (6.19) as

$$
\frac{d \sigma}{d \Phi_{n}^{(*)}}=\frac{d \sigma}{d \Phi_{1}^{(r)} d \Phi_{n-1}^{(f)}}=\frac{E_{r}}{E_{1}} \frac{d \sigma}{d \Phi_{n}^{(f)}},
$$

where $E_{r}$ is the energy of resonance $r$, and $d \Phi_{n-1}^{(f)}$ is the phase space factor for $\left(c_{2}, \cdots, c_{n}\right)$.

\subsubsection{Likelihood for an event}

To calculate the likelihood for an event, we start with the parton kinematics reconstruction $\boldsymbol{c}$ as follows:

(1) We assume momentum of $e$ or $\mu$ is precisely measured.

(2) Jets are assigned to quarks. We call this assignment "topology". We identify the momentum direction of each jet with that of identified quarks. Transfer variables $\boldsymbol{x}$ are chosen as quark energies $\left(E_{b}, E_{\bar{b}}, E_{q}, E_{\bar{q}^{\prime}}\right)$ and $\boldsymbol{y}$ as energies of the corresponding jets. The correspondence between $\boldsymbol{x}$ and $\boldsymbol{y}$ is topology dependent, hence we denote the transfer function by $w\left(I_{t}, \boldsymbol{y} \mid \boldsymbol{x}\right)$, where $I_{t}$ is the topology number. The transverse components of the neutrino $\left(\nu_{x}, \nu_{y}\right)$ are inferred from $\boldsymbol{E}_{\boldsymbol{T}}$ with compensation for jet energy inferences. 
(3) By (1) and (2), parton momenta are defined except for $\nu_{z}$, unmeasured $z$-component of the neutrino momentum. To get $\nu_{z}$ we infer $s_{W}$ according to $\Pi\left(s_{W}\right)$, and $\nu_{z}$ is obtained by solving Eq. (6.16). The two solutions $\left(\nu_{z 1}, \nu_{z 2}\right)$ are assigned with a solution number $I_{s}=1,2$. According to Eq. (6.22), the cross section is given by

$$
\frac{d \sigma}{d \Phi_{6}^{(*)}}=\frac{d \sigma}{d \Phi_{1}^{(W)} d \Phi_{5}^{(f)}}=\left(\frac{E_{W}}{E_{\nu}}\right)_{I_{s}} \frac{d \sigma}{d \Phi_{6}^{(f)}},
$$

where $d \Phi_{5}^{(f)}$ is the phase space of $\left(l, b, \bar{b}, q, \bar{q}^{\prime}\right)$.

(4) By (1), (2) and (3), a single kinematics path, i.e. a single topology and a single set of $\boldsymbol{x}$, is defined. For an inferred path, we scan $M_{t o p}$ in its search region.

The $k$-th event likelihood is then defined by the average of likelihood from all inferred paths,

$$
\boldsymbol{L}\left(M_{t o p} \mid \boldsymbol{y}^{(k)}\right)=l_{0} \frac{1}{N_{x}} \sum_{i=1}^{N_{x}} \sum_{I_{s}=1}^{2} \frac{d \sigma}{d \Phi_{6}^{(*)}} w\left(\boldsymbol{x}_{i} \mid I_{t}, \boldsymbol{y}^{(k)}\right)
$$

where $N_{x}$ is the total number of paths including jet- parton assignments. To obtain the top quark mass $M_{t o p}$ from a total of $N_{e v}$ events, we multiply the event likelihood functions each other, and define the following quantity,

$$
\boldsymbol{\Lambda}\left(M_{t o p}\right)=-2 \ln \left(\prod_{k=1}^{N_{e v}} \boldsymbol{L}\left(M_{t o p} \mid \boldsymbol{y}^{(k)}\right)\right) .
$$

Then the top quark mass is determined from the maximum likelihood estimator of $M_{\text {top }}$,

$$
\hat{M}_{t o p}=M_{t o p} \text { at minimum } \boldsymbol{\Lambda}\left(M_{t o p}\right) \text {. }
$$

\subsubsection{Matrix Element Calculation in the lepton+jets channel}

In this channel, the reconstruction path from observed $\boldsymbol{y}$ to parton momenta $\boldsymbol{c}\left(\boldsymbol{c}_{1}, \cdots, \boldsymbol{c}_{n}\right)$ are specified by the assignment of 4 jets to quarks $(b, \bar{b}, q, \bar{q})$, the value of $\boldsymbol{x}$, and two solutions for the neutrino longitudinal momentum $\left(\nu_{z}\right)$. All possible paths are mutually exclusive, and we define the i-th event likelihood as a sum of the likelihoods for all paths. Since we require at least one b-tagged jet, we consider 12 or 4 combinations for the reconstruction depending on whether 1 or 2 jets are b-tagged. The parton distribution functions $f_{a / p}\left(z_{1}\right)$ and $f_{b / \bar{p}}\left(z_{2}\right)$ are obtained from PYTHIA[40] using the leading order PDF, CTEQ5L. Other PDF sets are used as a systematic uncertainty. The transverse distribution function $f_{T}\left(p_{T}\right)$ is obtained by running the PYTHIA generator. 
The spin averaged matrix element squared $|\mathcal{M}|^{2}$ is factorized into 3 parts: (1) $t \bar{t}$ production $(\mid$ $\left.\left.\mathcal{M}_{t \bar{t}}\right|^{2}\right)$, (2) the propagators of the top and anti-top $\left(\left|\mathcal{P}_{t l}\right|^{2}\right.$ and $\left|\mathcal{P}_{t h}\right|^{2}$ ), and (3) the decay matrices for leptonic and hadronic top decays $\left(\left|\mathcal{D}_{t l}\right|^{2}\right.$ and $\left.\left|\mathcal{D}_{t h}\right|^{2}\right)$, where $t l$ and $t h$ indicate leptonic and hadronic decay channels of top respectively. Namely,

$$
|\mathcal{M}|^{2}=\left|\mathcal{M}_{t \bar{t}}\right|^{2}\left|\mathcal{P}_{t l}\right|^{2}\left|\mathcal{P}_{t h}\right|^{2}\left|\mathcal{D}_{t l}\right|^{2}\left|\mathcal{D}_{t h}\right|^{2}
$$

The production matrix element for the $q \bar{q}$ process is

$$
\left|\mathcal{M}_{t \bar{t}}(q \bar{q} \rightarrow t \bar{t})\right|^{2}=\frac{g_{s}^{4}}{9}\left(2-\beta^{2} \sin ^{2} \theta^{*}\right)
$$

where $\theta^{*}$ is the angle between the top quark and the incident quark/gluon in the proton in the $t \bar{t}$ center of mass system, $\beta$ is the velocity of the top and $g_{s}$ is the strong coupling constant.

For the $g g$ process, the matrix element can be expressed as

$$
\left|\mathcal{M}_{t \bar{t}}(g g \rightarrow t \bar{t})\right|^{2}=g_{s}^{4}\left(\frac{1}{6 \tau_{1} \tau_{2}}-\frac{3}{8}\right)\left(\tau_{1}^{2}+\tau_{2}^{2}+\rho-\frac{\rho^{2}}{4 \tau_{1} \tau_{2}}\right)
$$

where

$$
\tau_{1}=\frac{2(q \cdot t)}{\hat{s}}, \tau_{1}=\frac{2(\bar{q} \cdot t)}{\hat{s}}, \rho=\frac{4 M^{2}}{\hat{s}} .
$$

In these equations, the $t \bar{t}$ spin correlations for both the $q \bar{q}$ and $g g$ processes are averaged out, since correlations do not affect the mass measurement. Likewise, whether the $t \bar{t}$ is produced via $q \bar{q}$ or $g g$ has no effect on the mass measurement, hence the likelihoods for the two processes are summed up in the event likelihood with the appropriate PDF weight.

The decay matrix elements for the leptonic and hadronic channels are expressed as

$$
\begin{aligned}
\left|\mathcal{D}_{t l}\right|^{2} & =\frac{g_{w}^{4}}{4} \frac{(t \cdot l)(b \cdot \nu)}{\left(S_{l \nu}-M_{W}^{2}\right)^{2}+M_{W}^{2} \Gamma_{W}^{2}} \\
\left|\mathcal{D}_{t h}\right|^{2} & =\frac{g_{w}^{4}}{4} \frac{1}{2} \sum_{i \leftrightarrow j}^{2} \frac{\left(\bar{t} \cdot j_{i}\right)\left(\bar{b} \cdot j_{j}\right)}{\left(S_{2 j}-M_{W}^{2}\right)^{2}+M_{W}^{2} \Gamma_{W}^{2}}
\end{aligned}
$$

where $S_{l \nu}$ and $S_{2 j}$ represent the invariant masses squared of the lepton+neutrino and the 2jets from the $\mathrm{W}$ respectively. As for the mass and the decay width of $\mathrm{W}$, we assume the world average values, $M_{W}=80.4 \mathrm{GeV} / c^{2}$ and $\Gamma_{W}=2.1 \mathrm{GeV} / c^{2}$. Since the z-component of the neutrino momentum is not measured, $S_{l \nu}$ is inferred with $\Pi\left(s_{W}\right)$. The generated value of $S_{l \nu}$ gives the z-component of the neutrino momentum via Eq. (6.16). In Eq. (6.32), we make both possible assignments of the 2 jets to $q$ and $\bar{q}^{\prime}$ from the W, and the likelihoods corresponding to the two possibilities are averaged. 


\subsection{Transfer Function}

As described in the preceding Section, the transfer functions deal with relations between parton variables and observables. Since generic jet correction in the CDF experiments is based on the QCD process, we make further corrections for $b$ jets and $W$ daughter jets by using Monte Carlo generator for the process.

\subsubsection{Definition and Performance}

The transfer functions we use in this analysis are for energies of individual jets and corresponding partons. Thus variable set $(x, y)$ represents energies of a single parton and a jet. By running a Monte Carlo event generator with full detector simulation, we get a 2-dim histogram $D\left(x_{i}, y_{j}\right.$ : $\left.M_{t o p}\right)$, i.e. the number of events in bin $\left(x_{i}, y_{j}\right)$. In this analysis, we use Monte Carlo generators PYTHIA and HERWIG combined with CDF detector simulator. The distribution for $x$ of parton is given by

$$
n_{x i}=L_{i n t}\left(\frac{d \sigma}{d x}\right)_{i}=\sum_{j} D\left(x_{i}, y_{j}: M_{t o p}\right) .
$$

A distribution function defined by

$$
w\left(x_{i}, y_{j}: M_{t o p}\right)=\frac{1}{n_{x i}} D\left(x_{i}, y_{j}: M_{t o p}\right),
$$

correlates $x$ and $y$ without the cross section information, $d \sigma / d x$, and satisfies the normalization condition,

$$
\sum_{j} w\left(x_{i}, y_{j}: M_{t o p}\right)=1 .
$$

In practice, the transfer function $w(y \mid x)$ can be conveniently expressed as a function of variable $\xi$ as

$$
w\left(\boldsymbol{x} \mid \boldsymbol{y} \| M_{t o p}\right)=f(\xi) .
$$

We call variable $\xi$ a "response function" or a "transferred variable" in this paper and defined as

$$
\xi=\frac{E(\text { parton })-E(j e t)}{E(\text { parton })}
$$

where $E(j e t)$ is after generic corrections described in Section 3.2 and $E$ (parton) is a parton energy. This is done for $b$ jets and $W$ daughter jets separately.

As illustrated in figure 6.1 and figure 6.2, the T.F.'s strongly depend on Et of jets, and slightly depend on pseudo-rapidity $\eta$. Therefore we introduce these dependences by slicing Et of jets into 9 bins, from 15 to $>95 \mathrm{GeV}$ with $10 \mathrm{GeV}$ step(cut for jets: $E_{T}>15 \mathrm{GeV}$ ), and 
$|\eta|$ into 3 bins, 0.0-0.7-1.3-2.0(cut for jets: $|\eta|<2.0$ ) which correspond to different blocks of calorimeter tower geometry. ("Central", "Wall" and "Plug" region) [21].

The CDF has employed traditionally mean, median or MPV of the response distributions and fitted them by exponential functions with $p_{T}$ of the jets as a parameter [13, 47]. The distribution is, however, asymmetric due to mismatch between the jet clustering algorithm and hadronization. We do not fit the distribution with any functional form in this analysis, but generate random numbers according to "the raw distributions of variables" ("raw" means distributions themselves.)
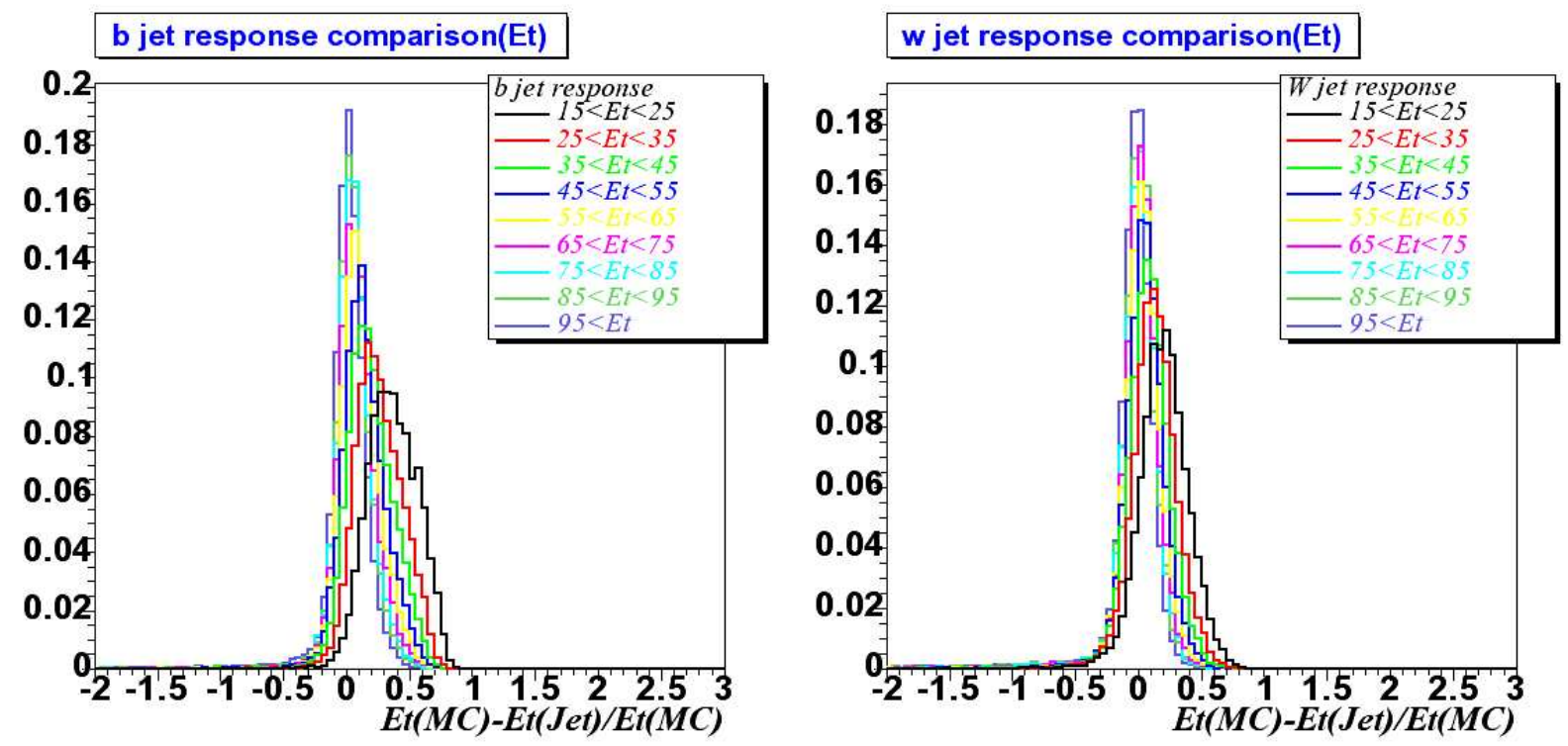

Figure 6.1: The response variables(transfer function) for $b$ jets(left) and $w$ jets(right) with different $E_{T}$ of the jets.

To validate the transfer function performance within Monte Carlo sample, We investigated the invariant mass of 2 jets from $\mathrm{W}$ and 3 jets from top quark by the following procedures.

1. jet-parton matching

Exclusive matching for jets and partons by requiring the distance $(\Delta R)$ between jets direction and partons direction is less than 0.4 to find correct correspondence between jets and partons. "Exclusive matching" means that if two or more jets are within $\Delta \mathrm{R}<0.4$ along a parton direction, the event matching is failed.

2. Applying the transfer function 

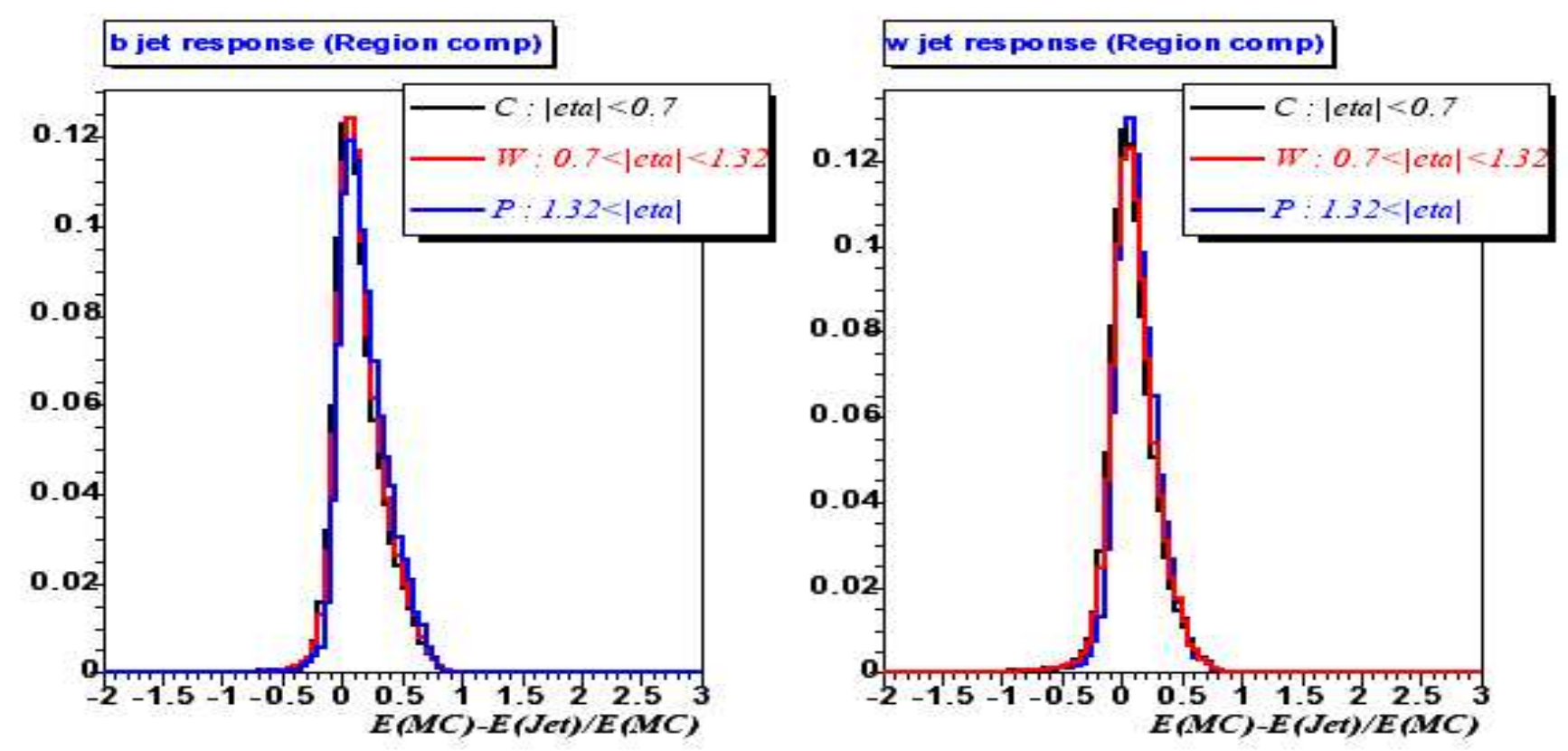

Figure 6.2: The response function in each detector $\eta$ of the jets bins for $b$ jets(left) and $w$ jets(right). Central $(<0.7)$ in black, Wall $(0.7<\eta<1.32)$ in red and Plug $(>1.32)$ in blue.

This is performed by random generations of the response variable along the each distribution. Energies of the partons are obtained by,

$$
E(\text { parton })=\frac{E(j e t)}{(1-\xi)}
$$

And then 2 jets(W) and 3jets(top) invariant mass are calculated. The random number generation is repeated more than $10 \mathrm{~K}$ times(we call it "scanning", Note that this is corresponding to integration of $\boldsymbol{x}$ in Eq. (6.24). After the scanning, distributions of 2jets/3jets invariant mass are obtained for each event.

3. Pick up the mean of the distribution obtained in procedure 2 by fitting the distribution in one event with Gaussian function. And fill the output mean value into the histogram.

The invariant masses of 2jets and 3jets before and after applying the transfer function can be shown in figure 6.3 and its mean and RMS are also summarized in Table 6.1. The mean value is reflecting the real (generated input) mass and resolutions are also improved after the transfer function. In addition, The left plots in figure 6.4 shows the detector $\eta$ dependence of those invariant masses, and $p_{T}$ dependence is shown in the right plots. There is a large $p_{T}$ 
dependence in the plots before the transfer function(right after applied generic jet correction), the transfer function however, eliminates it.
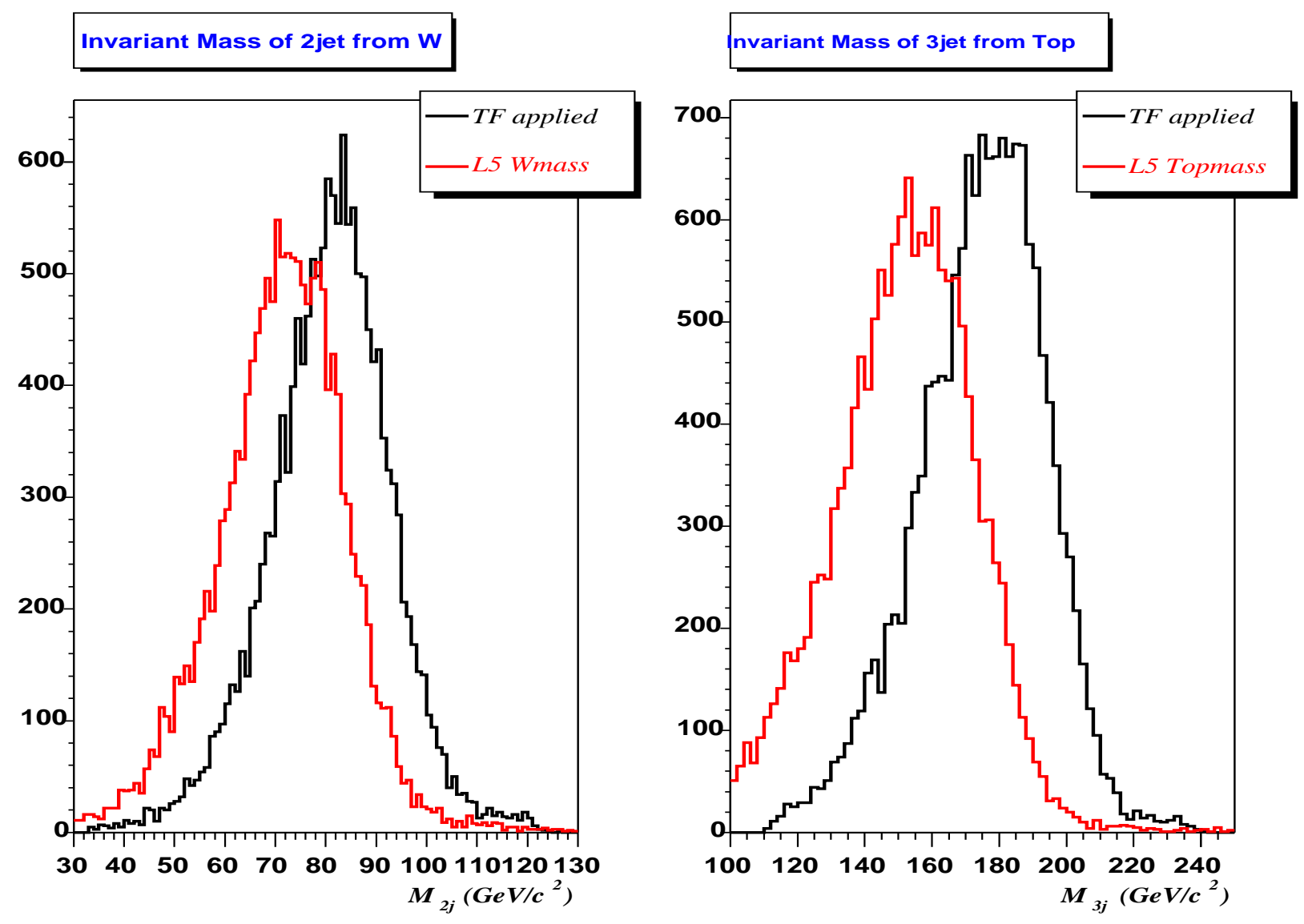

Figure 6.3: Comparisons of reconstructed 2 jets W(left) and 3 jets top(right) invariant masses before and after transfer function applied. Masses right after generic corrections are shown in black and red line corresponds to the ones after generic corrections plus transfer function.

\begin{tabular}{c|c||c|c}
\hline \hline \multicolumn{2}{c||}{ 2jets invariant mass of $\mathrm{W}\left(\mathrm{GeV} / c^{2}\right)$} & \multicolumn{2}{c}{ 3jets invariant mass of top $\left(\mathrm{GeV} / c^{2}\right)$} \\
\hline Before T.F. & After T.F. & Before T.F. & After T.F. \\
\hline$<71.4>12.8$ & $<80.9>12.2$ & $<152.1>20.4$ & $<174.8>19.1$ \\
\hline \hline
\end{tabular}

Table 6.1: The comparison of the 2jets/3jets mass of $\mathrm{W} /$ top before and after the transfer function applied. $<$ Mean $>$ and RMS are shown. 

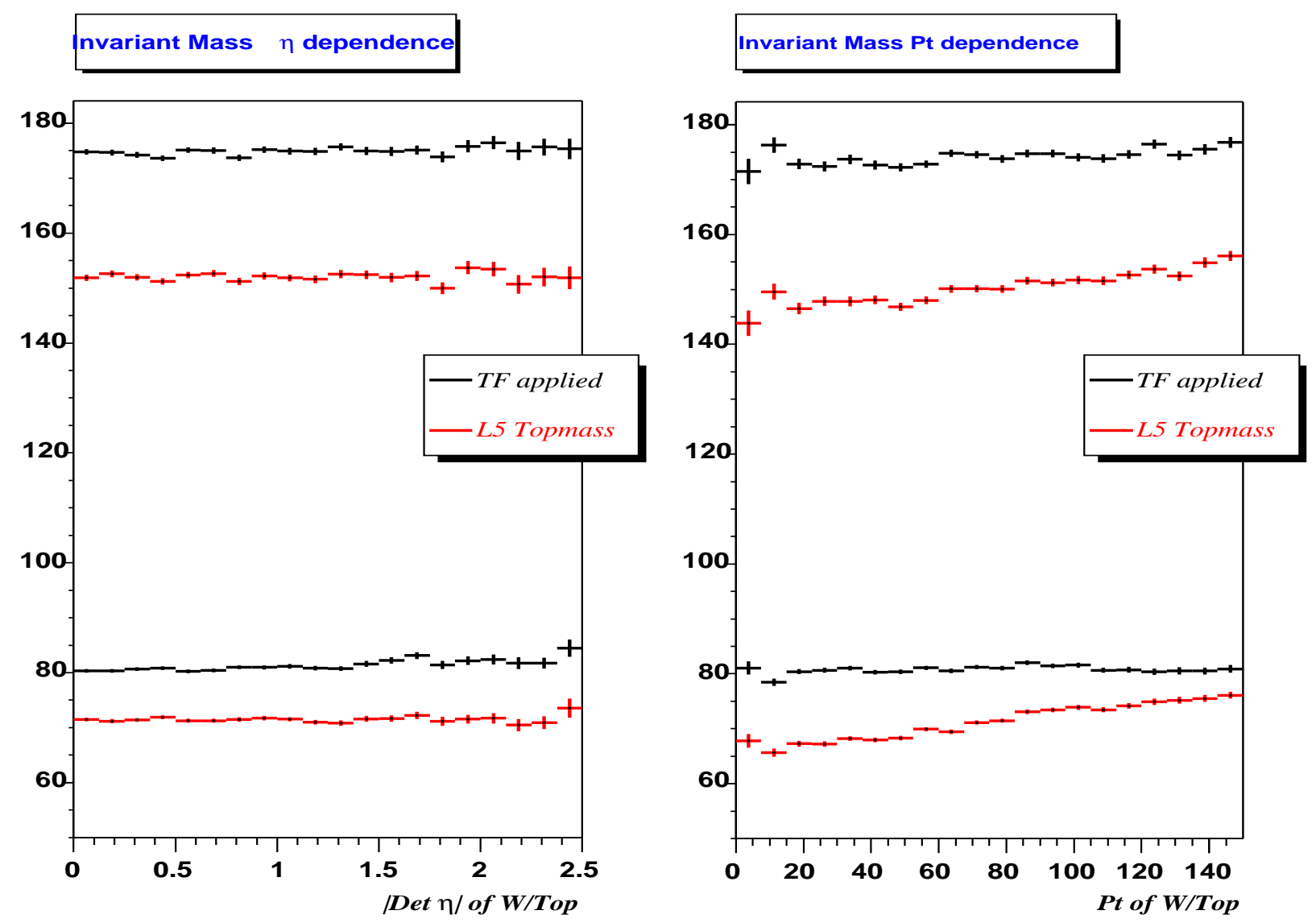

Figure 6.4: Comparisons of generic jet correction (black) and transfer function (red) in terms of reconstructed 2 jets $\mathrm{W}$ and 3 jets Top invariant masses: Detector $\eta$ of $\mathrm{W} /$ Top dependence(left) and $p_{T}$ of $\mathrm{W} /$ Top dependence(right).

We have investigated Monte Carlo dependence by comparing PYTHIA and HERWIG, even the fragmentation modeling is different between these two generators, but no significant discrepancies are observed. Since different top quark mass samples are made by HERWIG at CDF, we use the transfer function from PYTHIA as a default to avoid any unexpected bias within the generator. And we also have checked some variables to be used as a response function such as $E, E_{T}, p, p_{T}$ of the jets. Similar to the generator dependence, no differences are found in terms of the shape and mean of the response function and reconstructed invariant mass of 2 jets from $\mathrm{W}$ and 3jets from top quark. In DLM, the transfer functions described above are applied only to the four highest $E_{T}$ jets in the event, which are assumed to be coming from $t \bar{t}$ decays. 


\subsubsection{Missing Transverse Energy Treatments}

The "raw $\mathbb{E}_{T}$ " which is defined in Eq. (3.1) is corrected by two steps. The first step is for the generic jet energy corrections, and second is for the transfer functions. First, the definition of missing transverse energy can be rewritten using the observed objects in our sample to take into account the generic jet corrections as,

$$
-\boldsymbol{E}_{\boldsymbol{T}}=\boldsymbol{E}_{\boldsymbol{T}}(\text { lepton })+\sum_{i=1}^{4} \boldsymbol{E}_{\boldsymbol{T}}^{\boldsymbol{i}}(\text { jets })+\boldsymbol{X}_{\boldsymbol{T}}
$$

where $\boldsymbol{E}_{\boldsymbol{T}}^{i}$ (jets) is $E_{T}$ of the generic corrected jets and $\boldsymbol{X}_{\boldsymbol{T}}$ corresponds to all other calorimeter deposited energies.(within $\boldsymbol{X}_{\boldsymbol{T}}$, if there are jets satisfying $E_{T}>8 \mathrm{GeV}$ and $|\eta|<2.5$, the generic corrections are applied to those jets.) The above expression shows that the $\mathscr{E}_{T}$ measurement is highly correlated with the jet energy measurements and corrections. Therefore, it is not considered as an independent measurement in this analysis. To obtain the transverse component of the neutrino momentum $\boldsymbol{\nu}_{\boldsymbol{T}}$ from leptonic W decay, we recalculate it as follows,

$$
\boldsymbol{\nu}_{T}=\boldsymbol{E}_{T}+\sum_{j=1}^{4}\left(\boldsymbol{E}_{\boldsymbol{T}}^{j}(\text { corr })-\boldsymbol{E}_{\boldsymbol{T}}^{j}(\text { jets })\right)
$$

where $\boldsymbol{E}_{\boldsymbol{T}}^{j}$ (jets) and $\boldsymbol{E}_{\boldsymbol{T}}^{j}$ (corr) are $E_{T}$ of the jets after generic jet corrections and $E_{T}$ of the jets after generic and transfer function correction applied to leading four jets, respectively.

\subsection{Performances of the Method}

This section describes the top quark mass reconstructions for $t \bar{t}$ signal events and backgrounds events based on Monte Carlo simulation. In DLM algorithm in this analysis, all events are assumed to be signal and the likelihood is calculated. Therefore we first present the mass reconstruction using only signal events, and afterward, background effects are investigated. Lastly we derive the correction function to obtain the top quark mass by parameterizing a correction factor as a function of the fraction of backgrounds.

\subsubsection{Tests using Signal Monte Carlo Simulation}

The method is first validated by using only HERWIG $t \bar{t}$ sample with an input top quark mass of $175 \mathrm{GeV} / c^{2}$. Fig 6.5 shows the pull distribution from 2000 sets of 22 events pseudo experiment of reconstructed top quark mass. The variable "pull" is defined as,

$$
p u l l=\frac{M_{r e c}-M_{i n p u t}}{\sigma}
$$


where $M_{r e c}$ and $M_{\text {input }}$ are reconstructed top mass (output of DLM) and generated top mass (input of the sample) respectively. If $M_{r e c}>(<) M_{\text {input }}, \sigma$ is defined as $\sigma^{-(+)}$. In the case of an asymmetric uncertainty, variable pull is symmetries properly. The Gaussian fit to the pull distribution gives a mean of $-0.05 \mathrm{GeV} / c^{2}$. And the width is 1.01 which is consistent with a unit.

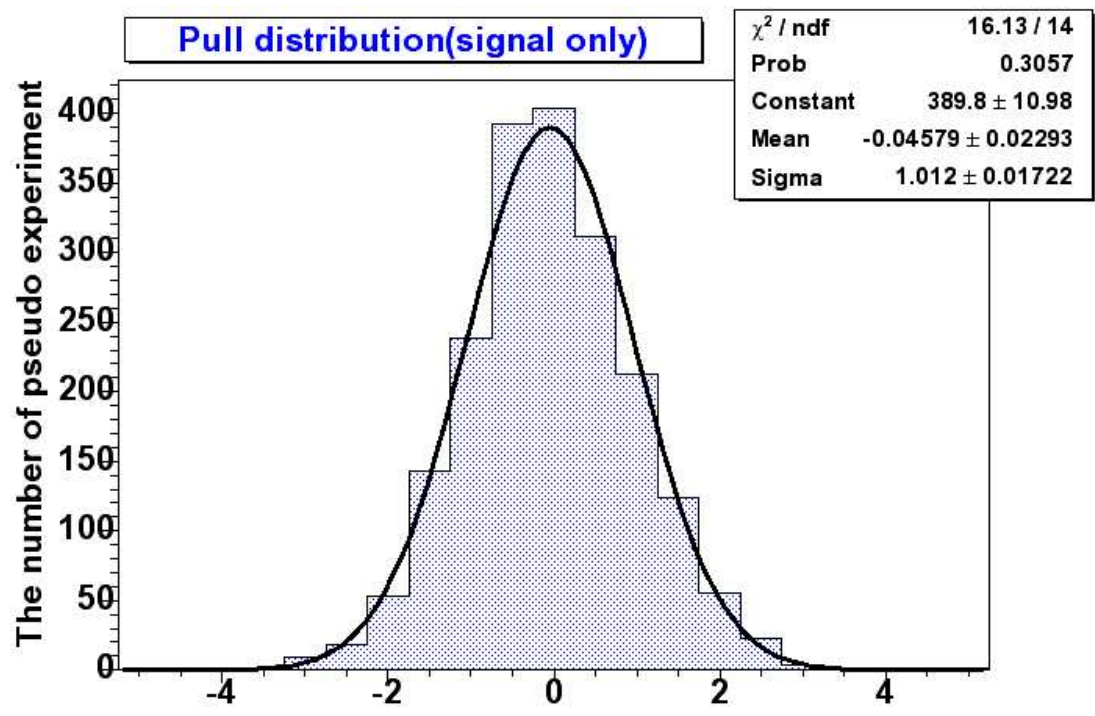

Figure 6.5: Pull distribution from 2000 sets of 22 events pseudo experiment of $t \bar{t}$ sample. Gaussian fit returned the mean of -0.05 and the sigma of 1.01 .

We try to determine the top quark mass with different top quark mass samples in the range of $[150-200] \mathrm{GeV} / c^{2}$ from HERWIG simulation. Figure 6.6 shows the reconstructed top quark mass as a function of input mass. Ideally, the slope has to be 1.0. Our results unfortunately returned the slope of 0.84 (red line in figure 6.6). We consider that the non-unit-slope is due mainly to the transfer functions, since we applied the transfer function for $M_{t o p}=175 \mathrm{GeV} / c^{2}$ PYTHIA to samples with different masses. In fact, the slope of the blue plots in figure 6.6, which was obtained by using the transfer functions from each mass samples, is 0.98. As we remarked before, the statistics of different mass samples are too limited to obtain a complete set of the mass-dependent transfer functions. In this paper, we use the transfer function from $M_{\text {top }}=175 \mathrm{GeV} / c^{2}$ sample alone. We do not think that this creates a critical problem or any bias for the reconstructions, if we use the non-unit-slope(red) as a mapping function to correct mass shift because we know how much the top quark mass is shifted due to transfer functions. 
As a matter of fact, figures 6.7 and 6.8 show sanity check and width of pull distributions from pseudo experiments as a function of input top quark mass after correcting mass shift by the mapping function(slope of 0.84 ). Center of pull distribution is consistent with 0.0 and its width is 1.0, hence we can reconstruct the top quark mass correctly in the wide range of input top quark mass[150-200] GeV $/ c^{2}$. Furthermore, as we see in the next section, backgrounds also pull down the output mass, even if we made the slope of 1.0. Thus we integrate the two effects, mass-dependence of the transfer function and the backgrounds, into a single mapping function described in Section 6.4.3 in this paper.

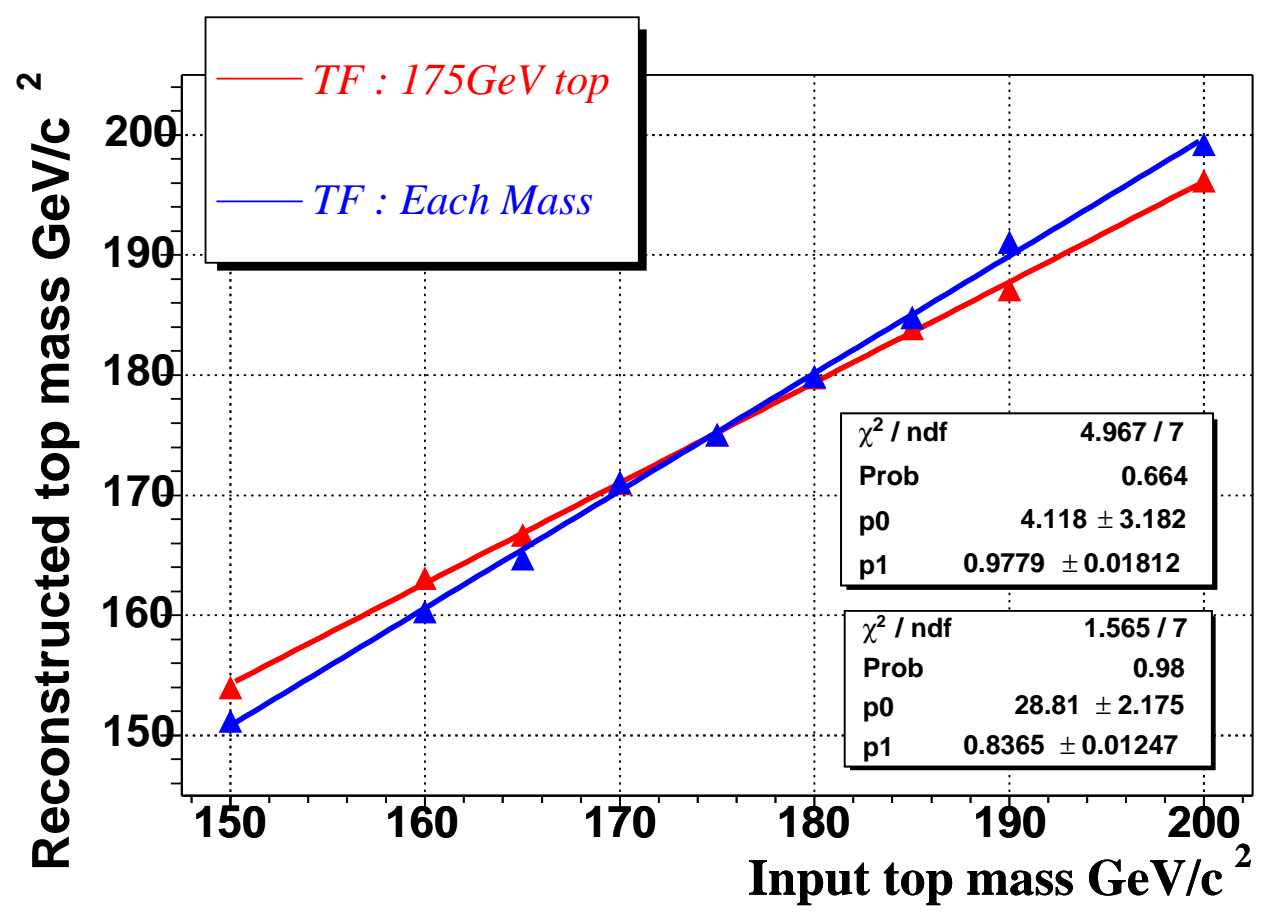

Figure 6.6: The reconstructed top quark mass with different mass samples. The linear fit in red is from applying the transfer function of $M_{t o p}=175 \mathrm{GeV} / c^{2}$ to all mass samples. Blue line shows the results using the transfer function obtained from each mass samples. The slopes are 0.84 and 0.98 for red and blue fit respectively.

\subsubsection{Backgrounds Effect on the Likelihood}

We use the ALPGEN Monte Carlo with CDF detector simulation to model mistags, $\mathrm{W}+$ heavy flavor events. $\mathrm{W}+$ four light flavor partons (W4p) process can be used for mistags since the 


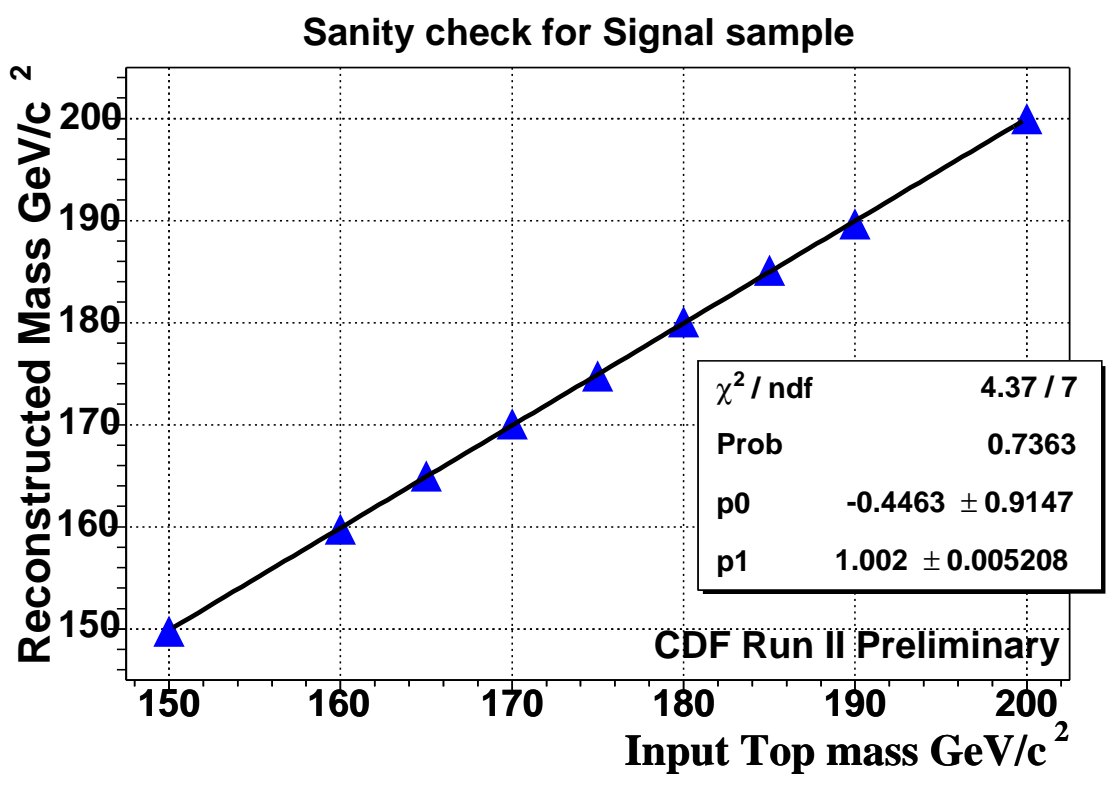

Figure 6.7: Sanity check. Reconstructed top quark mass as a function of its input top quark mass after taking into account mass shift by mapping functions.

mistags come from the false secondary vertex which is mainly due to track and vertex resolution. For QCD non-W background, we use non-isolated lepton sample(isolation $I>0.2$ but $\not_{T}>20$ $\mathrm{GeV}$ ) from real data. WW and signle top backgrounds are modeled by PYTHIA Monte Carlo samples. All events are used after event selection described in Section 4.3.

The likelihood distribution and the mass-likelihood peak are expected to be changed by the existence of background events. To understand the background effects more closely, we first calculate the dynamical likelihood defined by Eq. (6.24) for each background sample. Joint likelihood maximum peaks are summarized in Table 6.2. The maximum points are mainly from lepton and jets energy cut thresholds. Mistag, Wbb̄2p and QCD samples give almost the same maximum likelihood point near the lower limit of the search region, while the single top sample has slightly higher mass and Wcc, Wc and WW have slightly lower mass. Each background has no characteristic reconstructed mass in the search mass range, differently from the case of $t \bar{t}$.

To investigate the effect of backgrounds on $t \bar{t}$ signal events, figure 6.9 illustrates reconstructed top quark mass from 22 events pseudo experiments with varying the background fractions to look at how signal reconstructed mass $\left(M_{t o p}=175 \mathrm{GeV} / c^{2}\right)$ is shifted by the background. We can see the peak is shifted lower and the sigma is also larger as background fraction gets higher. 


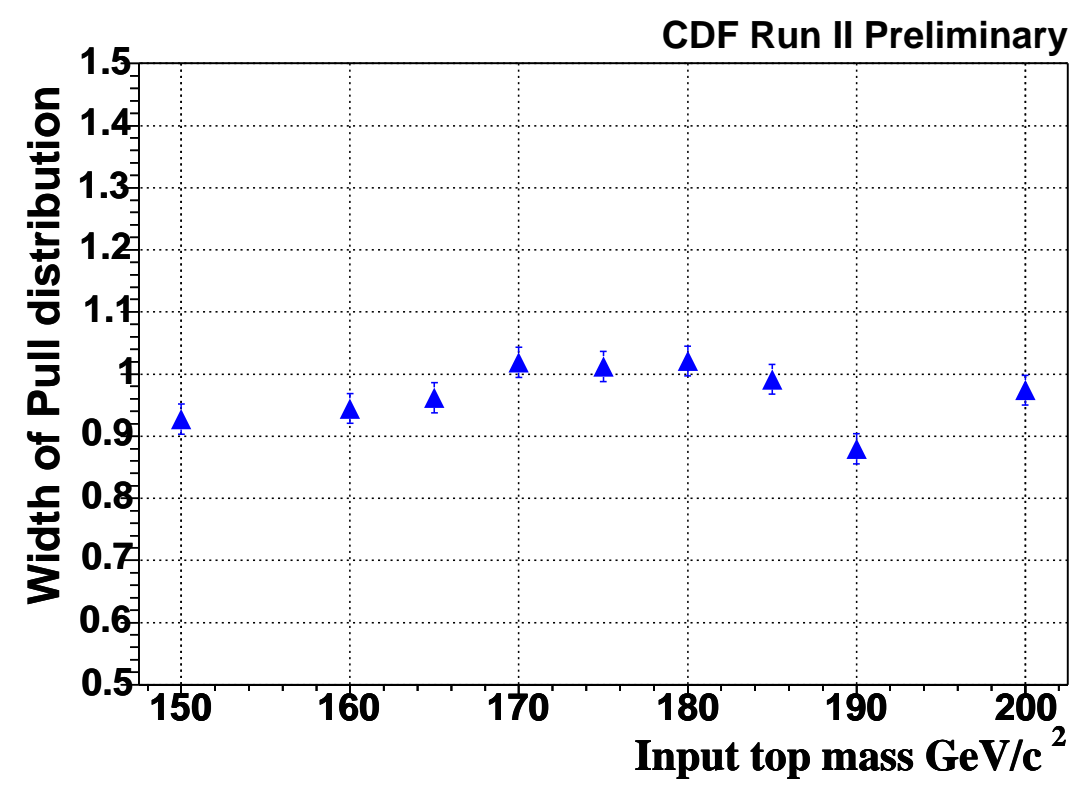

Figure 6.8: Width of Pull distribution as a function of input top quark mass after taking into account mass shift, which is consistent with a unit.

\begin{tabular}{c|c}
\hline \hline Source & Maximum likelihood point \\
\hline Mistag(W4p) & $\sim 162 \mathrm{GeV} / c^{2}$ \\
Wbb & $\sim 162 \mathrm{GeV} / c^{2}$ \\
Wc $\bar{c}$ & $\sim 157 \mathrm{GeV} / c^{2}$ \\
Wc & $\sim 158 \mathrm{GeV} / c^{2}$ \\
QCD(nonW) & $\sim 161 \mathrm{GeV} / c^{2}$ \\
Single Top & $\sim 170 \mathrm{GeV} / c^{2}$ \\
WW & $\sim 160 \mathrm{GeV} / c^{2}$ \\
\hline \hline
\end{tabular}

Table 6.2: The maximum likelihood masses for various background sources. 


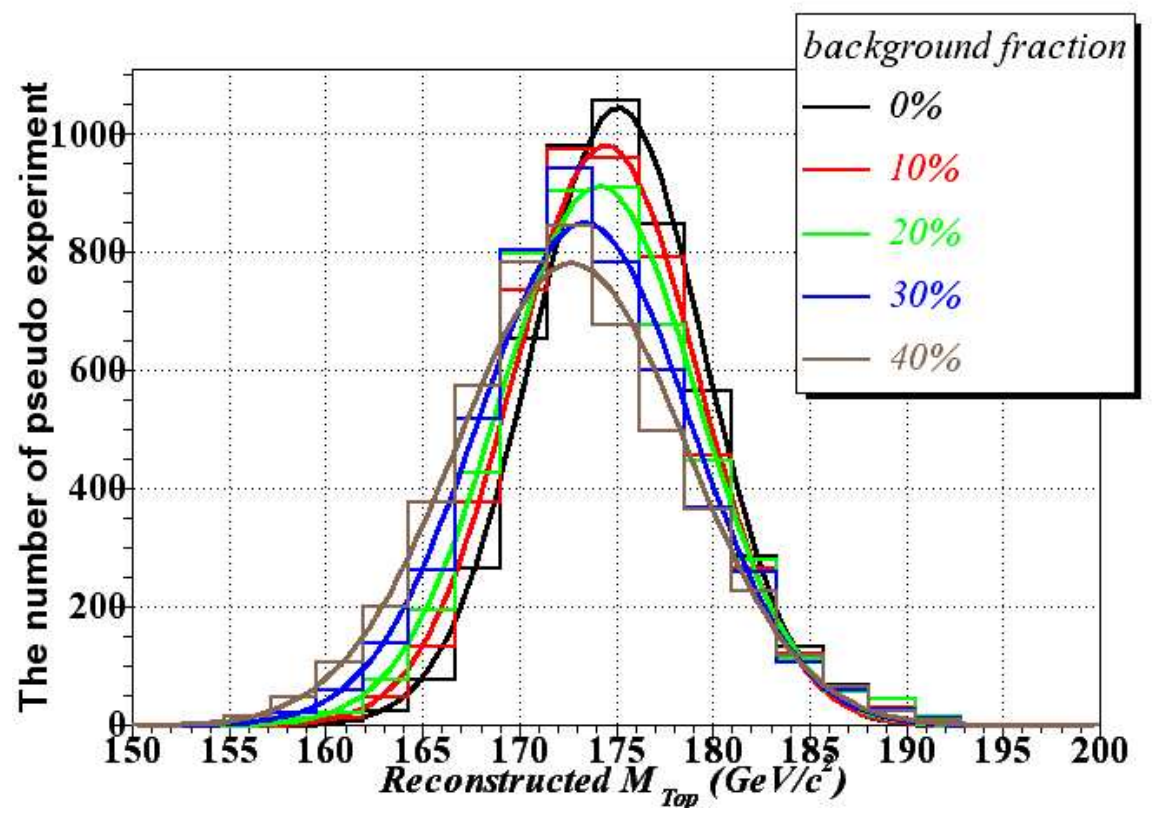

Figure 6.9: The reconstructed mass (input $M_{t o p}=175 \mathrm{GeV} / c^{2}$ ) with varying the background fraction between $0 \%$ to $40 \%$. Each entry contains 22 events for pseudo experiments.

In addition, it is good to know the effect of each of the backgrounds on the mass determination of the signal sample. Figure 6.10 shows the signal $t \bar{t}\left(M_{t o p}=175 \mathrm{GeV} / c^{2}\right)$ reconstructed mass shifted by individual background contaminations as a function of background fraction from [0-40]\% by pseudo experiment of 22 total number of events and the number of background events are fluctuated by Poisson function. We do not see large differences between the effects of $\mathrm{W} b \bar{b} 2 \mathrm{p}$, of mistag and of $\mathrm{QCD}($ nonW) samples which are more than $80 \%$ of the background. Hence all combined (solid red line in figure 6.10) is dominated by these three sources. From this result, the shift rate by the background is not sensitive to the relative fraction of Wbb $2 \mathrm{p}$, mistag and QCD(nonW). On the other hand, single top sample makes relatively smaller shift and Wcc/Wc/WW make larger shift than our dominant samples of mistag/Wbb/QCD. This is all consistent with the results in Table 6.2.

In summary, the backgrounds pull down the likelihood peak, and we need to evaluate the size of mass shifts systematically in order to extract true top quark mass. This correction is discussed in the next section as a mapping function. 


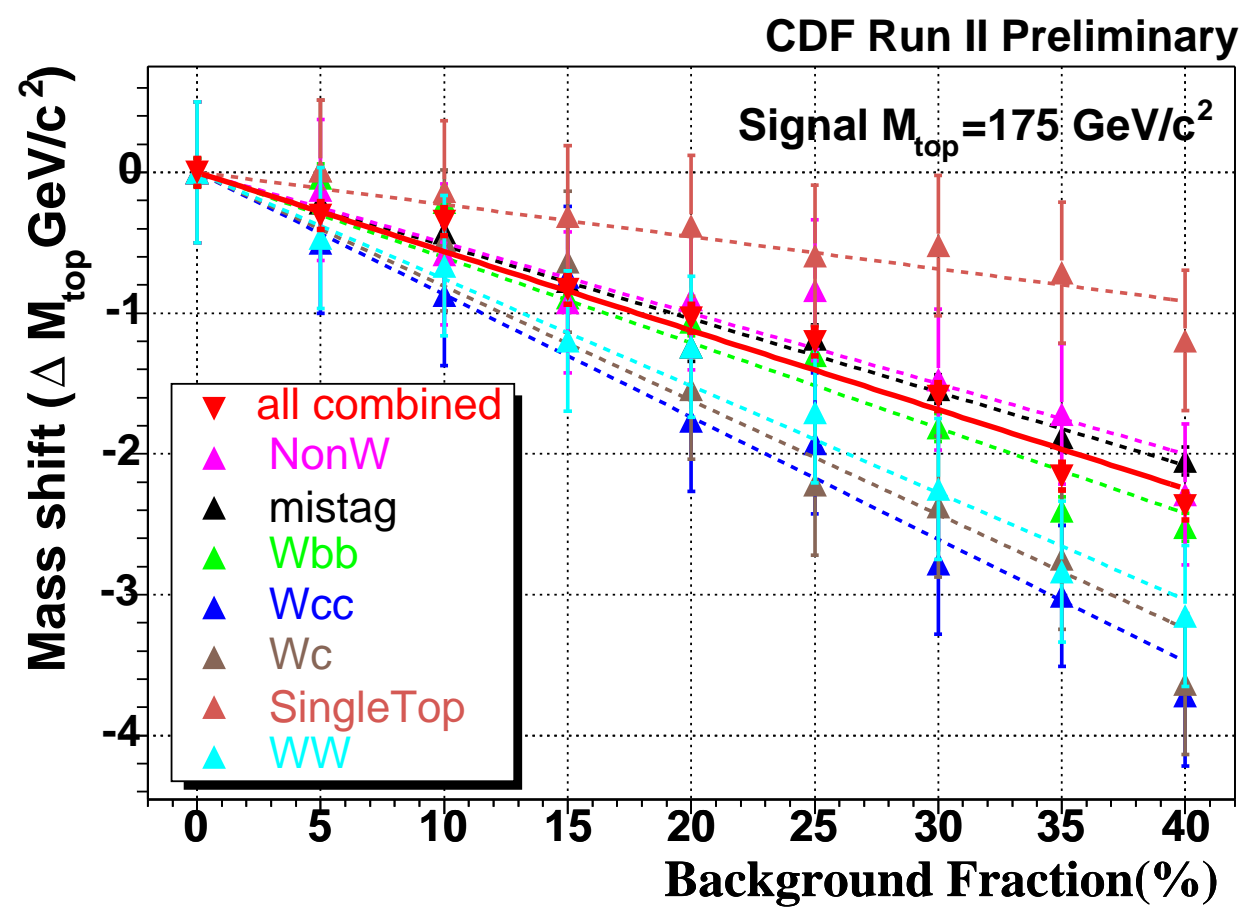

Figure 6.10: Mass shift $\left(M_{r e c}-M_{\text {input }}\right)$ due to individual background source using signal sample of $M_{\text {top }}=175 \mathrm{GeV} / c^{2}$ as a function of background fraction. Solid red line represents all combined background with expected number of events in Table 5.6.

\subsubsection{The mapping function to extract the top quark mass}

The mass-dependent correction factor, which we call the mapping function, is obtained with 2000 sets of pseudo-experiments. There are two sources that cause a non-unit-slope between the input top quark mass and the reconstructed top quark mass. One is the top quark mass dependence of the transfer function, and the other is the effect of background. We integrate the two effects into a single mapping function. Figure 6.11 shows the reconstructed top quark mass as a function of its input mass with various background fractions. The background fraction is changed from $0 \%$ to $40 \%$, where the relative fraction of each background source is Poisson fluctuated using the mean numbers given in Table 5.6 and the total number of events is fixed to 22 . As one can see in the figure, even with $0 \%$ background the reconstructed top quark mass does not have unit slope. Since we use only the transfer function from $M_{t o p}=175 \mathrm{GeV} / c^{2}$ in the present analysis, the mass-dependence of the transfer function is taken into account by this slope. In addition, we assume all events are $t \bar{t}$ candidates. If background events are included in the 
data sample, the likelihood peak is pulled down by these background events, since we multiply the event likelihoods. The reconstructed top quark mass is shifted lower as the background fraction increases. Figure 6.12 shows that the slope of linear fit in figure 6.11 as a function of background fractions. One can see very stable behavior up to background fraction of $40 \%$. Our estimated background fraction of approximately $19 \%$ is used to extract the top quark mass.

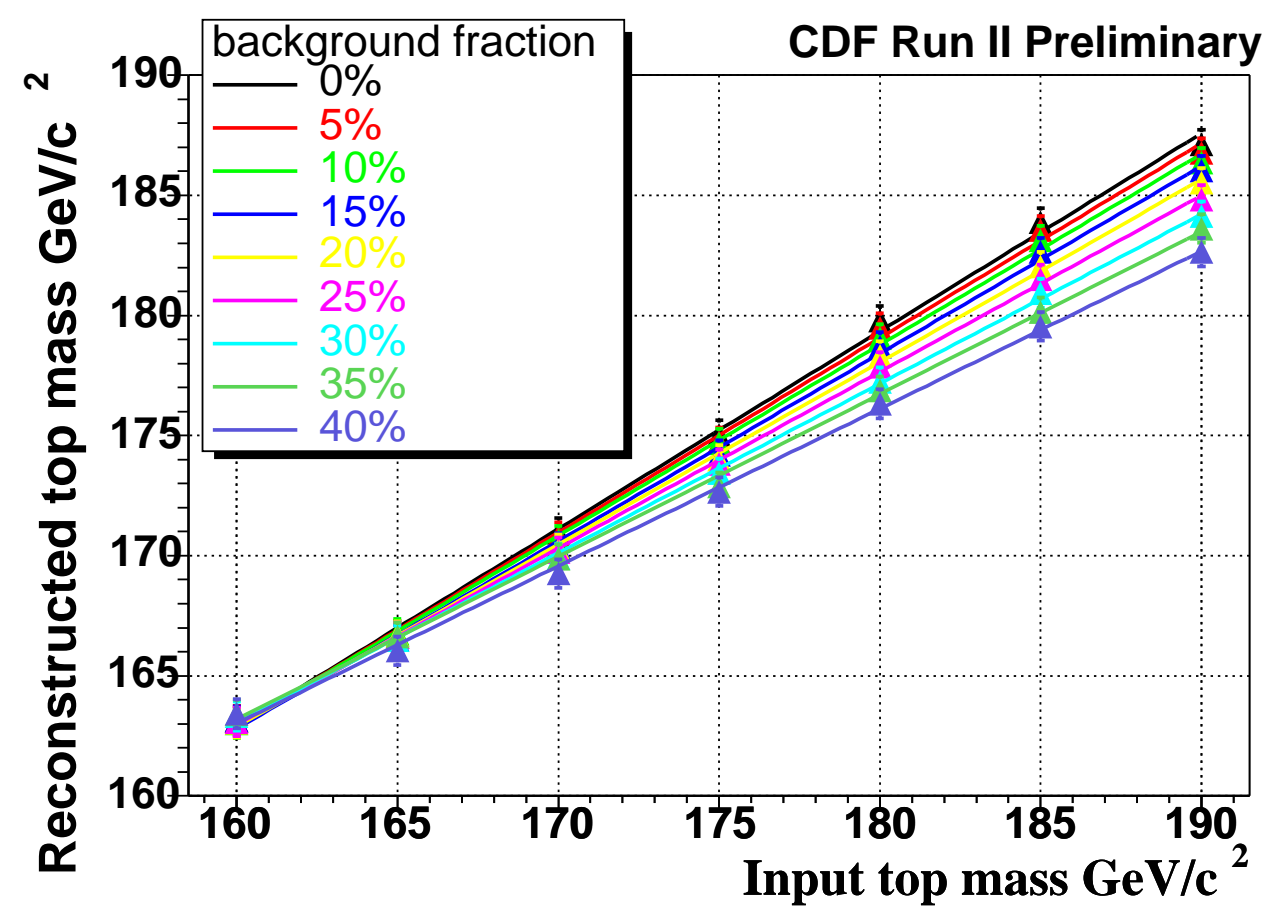

Figure 6.11: The reconstructed mass as a function of input mass with the background fraction from $0 \%$ to $40 \%$.

\subsubsection{Sanity check: mass pull distribution after using the mapping functions}

Each set of 22 events in a pseudo-experiment consists of on average 17.8 signal events and 4.2 background events, with each background source Poisson fluctuated. After applying mapping functions for a $19 \%$ background fraction to each pseudo-experiment, we obtain a slope of 1.0 between the input and reconstructed masses as illustrated in figure 6.13 (sanity check). The width of the pull distribution as a function of input top quark mass is shown in figure 6.14. We conclude that the top mass is reconstructed without bias over a wide range of input masses by applying the mapping function. 


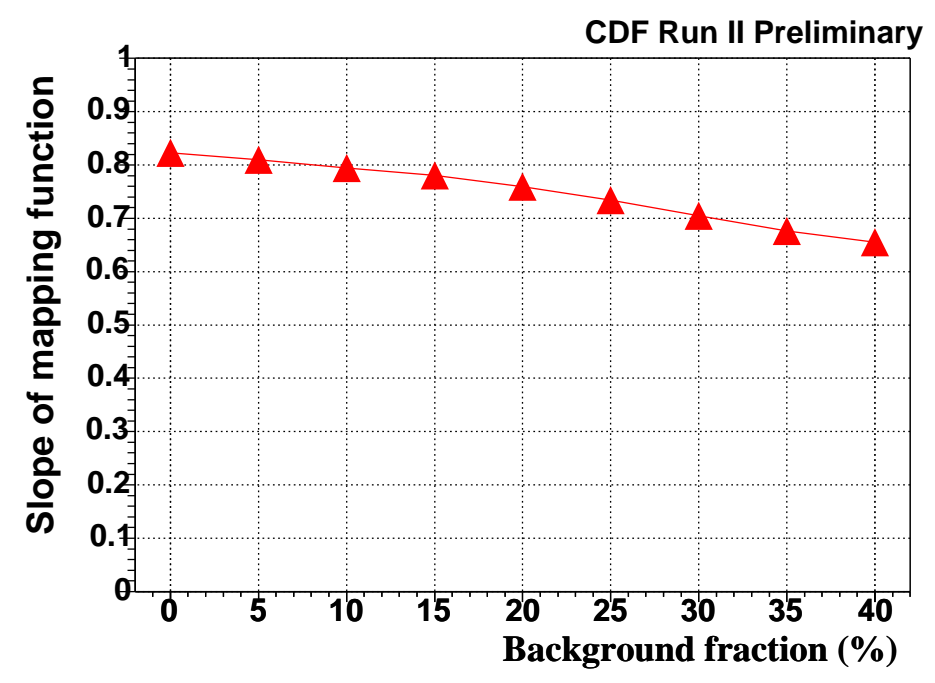

Figure 6.12: The slope of linear fit in figure 6.11 as a function of the background fraction. This can be used as a mapping function to extract the top quark mass from background pulling.

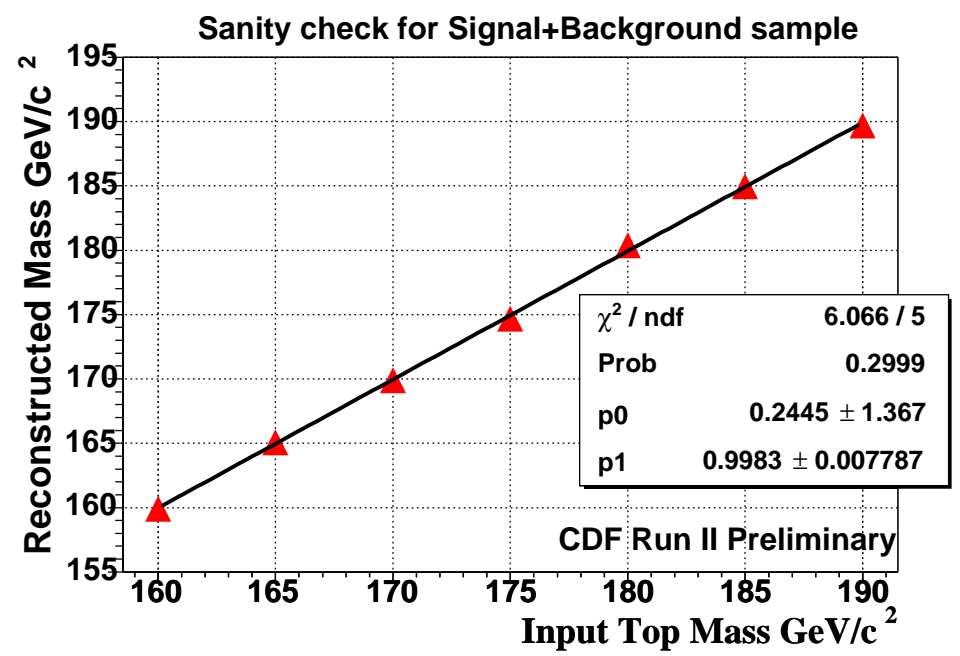

Figure 6.13: Sanity check after applying the mapping functions with a background of $19 \%$. The slope of the linear fit between the reconstructed top quark mass and its input mass is consistent with 1.0 


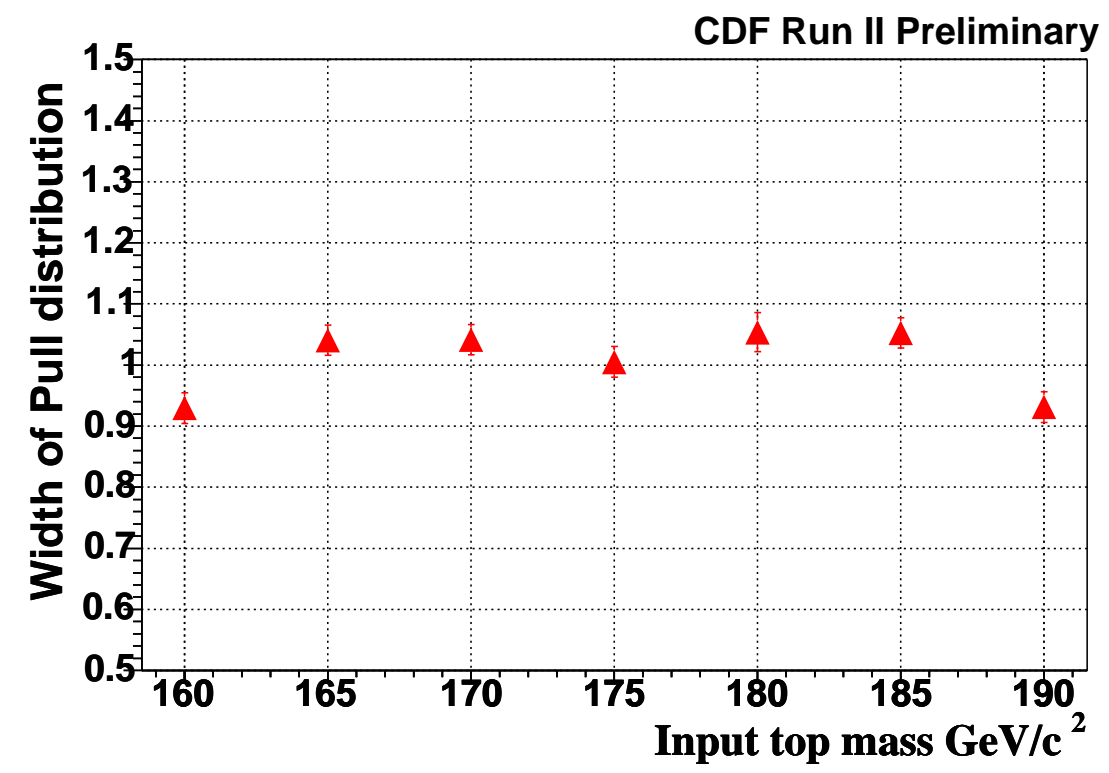

Figure 6.14: The width of the mass pull distribution, which is consistent with 1.0. 


\subsubsection{Blind Test Results}

There exist several techniques and more than 30 people have tried to measure the top quark mass at CDF. To invesitgate each analysis method, The Top Quark Mass Working Group [62] had generated six "blind samples". Blind sample by definition, blinds its input (true) top quark mass. Users were not informed input top masses and which Monte Carlo generator was used for those samples. Users were informed that only input top quark masses are in a reasonable range which is between 130 and $220 \mathrm{GeV} / \mathrm{c}^{2}$ and the samples contain only signal $t \bar{t}$ events. Each sample contains two hundred thousand events and there were about four thousand events available after event selections that is statistically enough to determine the top quark mass. Figure 6.15 shows the results of DLM. As one can clearly say, DLM has no biases in the wide range of input top quark mass. Since generator and the parton distribution function (PDF) employed for this samples were unknown, results were allowed to have offsets at the level of systematic uncertainty due to generator difference and PDF, which was estimated to be about $2 \mathrm{GeV} / c^{2}$ (see Section 8). All results are however, found to be consistent with input top masses within $1 \mathrm{GeV} / c^{2}$.

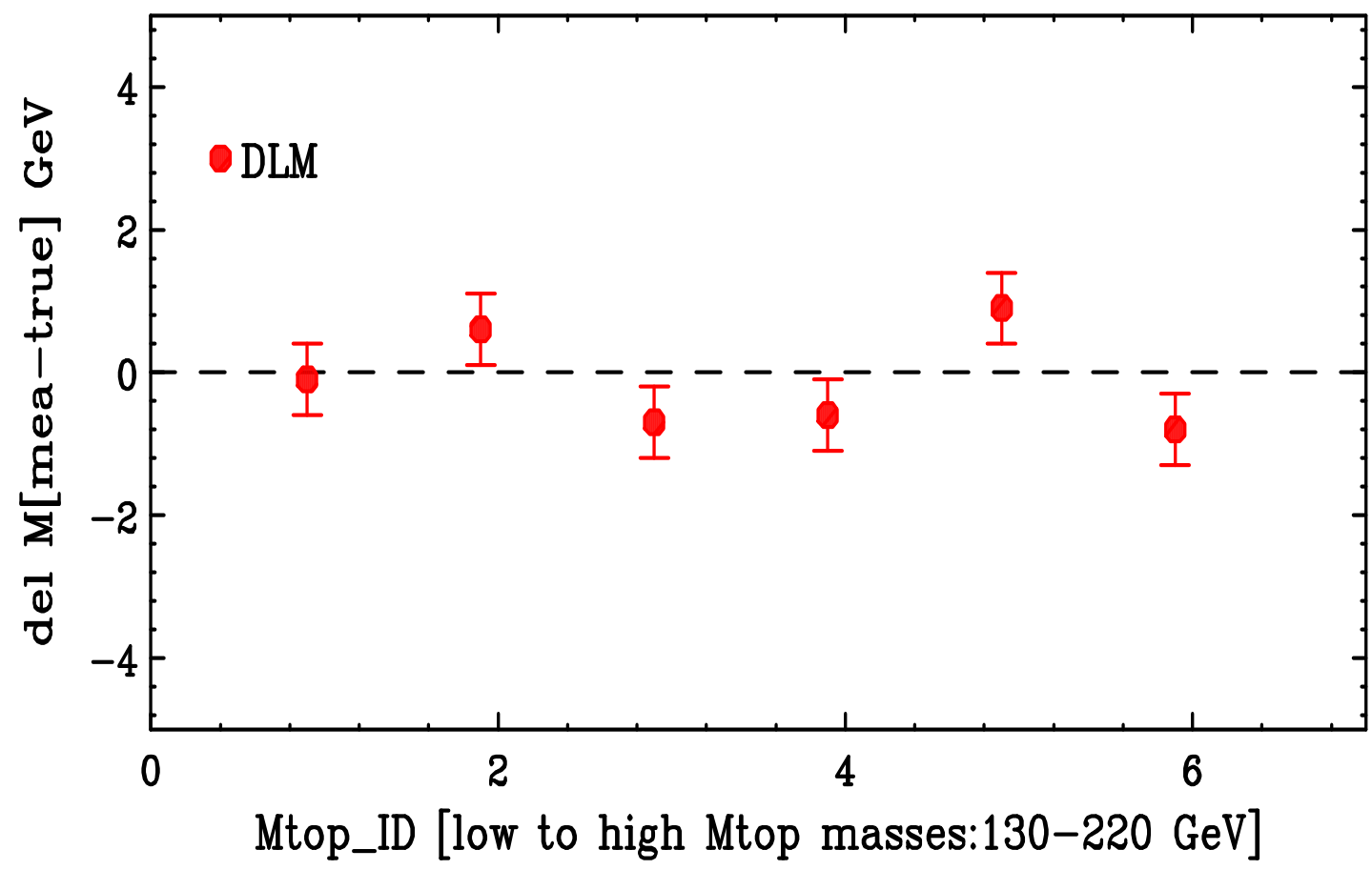

Figure 6.15: The results of blind tests. Plots are $\Delta M=M_{r e c}-M_{\text {true }}$ from low to high top quark mass. 


\section{Chapter 7}

\section{Results}

\subsection{Extracting Top Quark Mass}

We have twenty-two $t \bar{t}$ candidates in which twelve events have an electron, the rest is muon channel, passing our event selection criteria using an integrated luminosity of $162 \mathrm{pb}^{-1}$ taken at CDF Run II. 4.2 events are estimated as backgrounds (see Section 5.3). As previously described, each event contains one central isolated lepton (electron or muon) and a large missing transverse energy indicating an existence of a neutrino from $\mathrm{W}$ boson, and in our case, exactly four jets. A likelihood is calculated as a function of top quark mass for event by event, then an each event likelihood is multiplied each other to extract the top quark mass. Figure 7.1 shows the result of the joint likelihood of these events. It is fitted with a second order polynomial. From the fit, we obtain $M_{\text {top }}=176.5_{-3.8}^{+3.4}$ (stat.only) $\mathrm{GeV} / \mathrm{c}^{2}$, assuming there is no background. We apply the mapping function to remove the mass-pulling effect of the background. Figure 7.2 shows the extracted top mass as a function of the background fraction. The top quark mass changes by less than $1.5 \mathrm{GeV} / c^{2}$ in the background fraction range of [0\%-20\%].

For the final result, we use the estimated $19 \%$ background fraction, which gives $M_{t o p}=$ $177.8_{-5.0}^{+4.5}$ (stat.only) $\mathrm{GeV} / c^{2}$. Note that the statistical uncertainty must be scaled by the slope of the mapping function mass shift. Figure 7.3 shows a change of the statistical uncertainties as a function of background fraction. $+3.4(-3.8) \mathrm{GeV} / \mathrm{c}^{2}$ uncertainty has to be scaled up to $+4.5(-5.0) \mathrm{GeV} / \mathrm{c}^{2}$ at a background fraction of $19 \%$. About $32 \%$ scaling is due to effects from transfer function $\left(\mathrm{M}_{\text {top }}=175 \mathrm{GeV} / \mathrm{c}^{2}\right)$ and backgrounds, corresponding a mapping slope of 0.76 
in figure 6.12 .

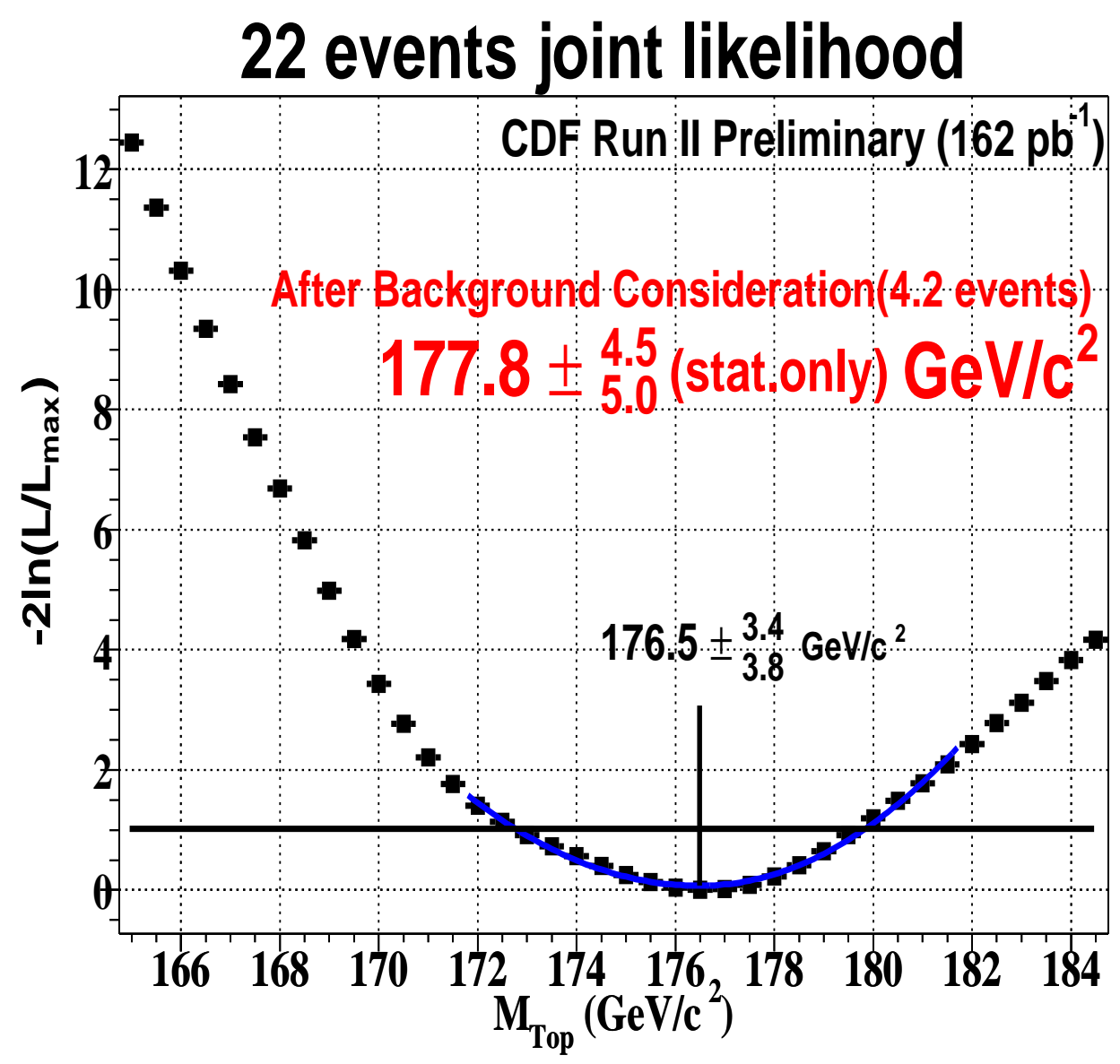

Figure 7.1: The joint negative log likelihood distribution of the 22 events gives $M_{t o p}=176.5_{-3.8}^{+3.4}$ $\mathrm{GeV} / \mathrm{c}^{2}$ 


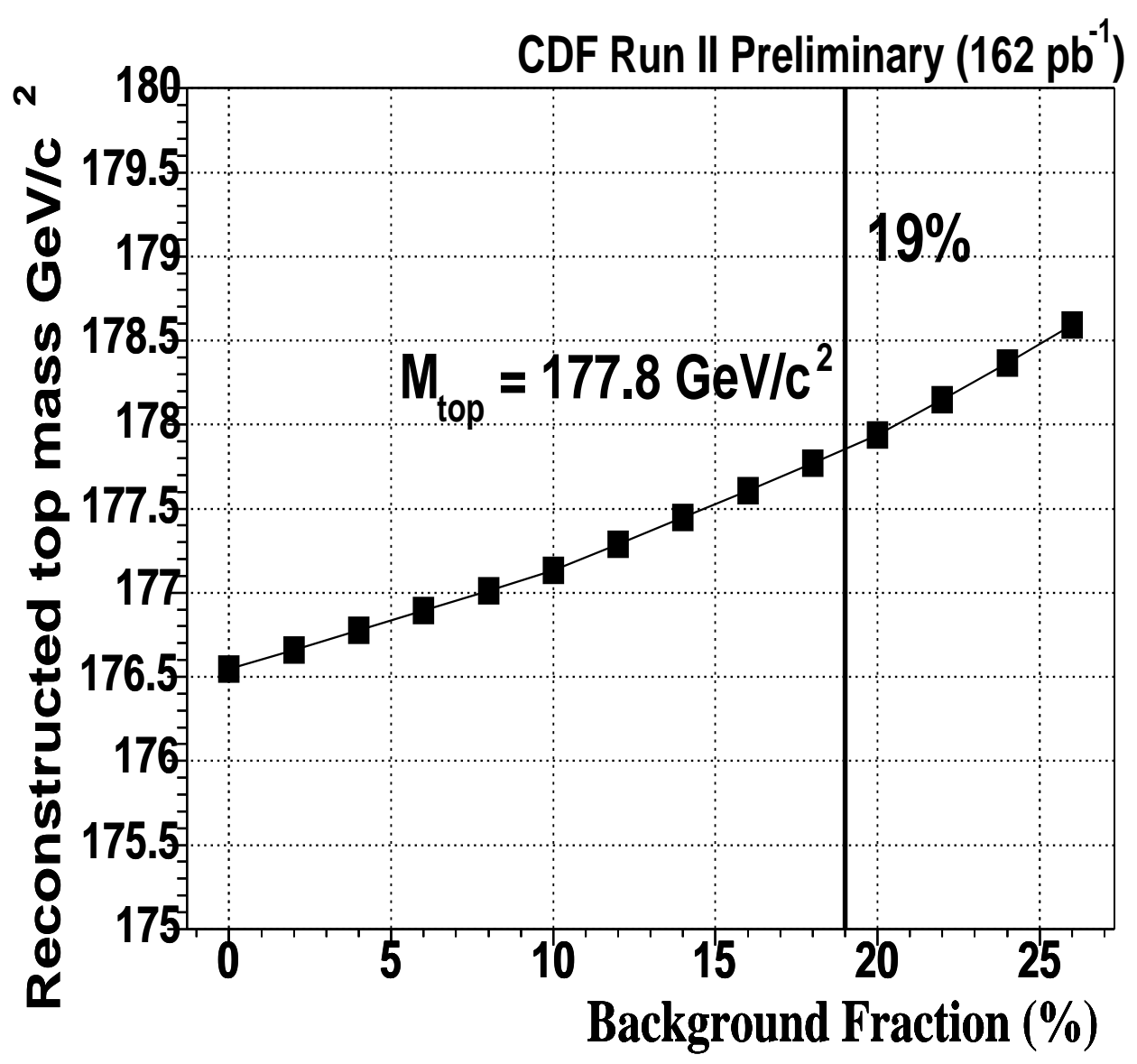

Figure 7.2: Extracted top quark mass using the mapping function as a function of the background fraction. 


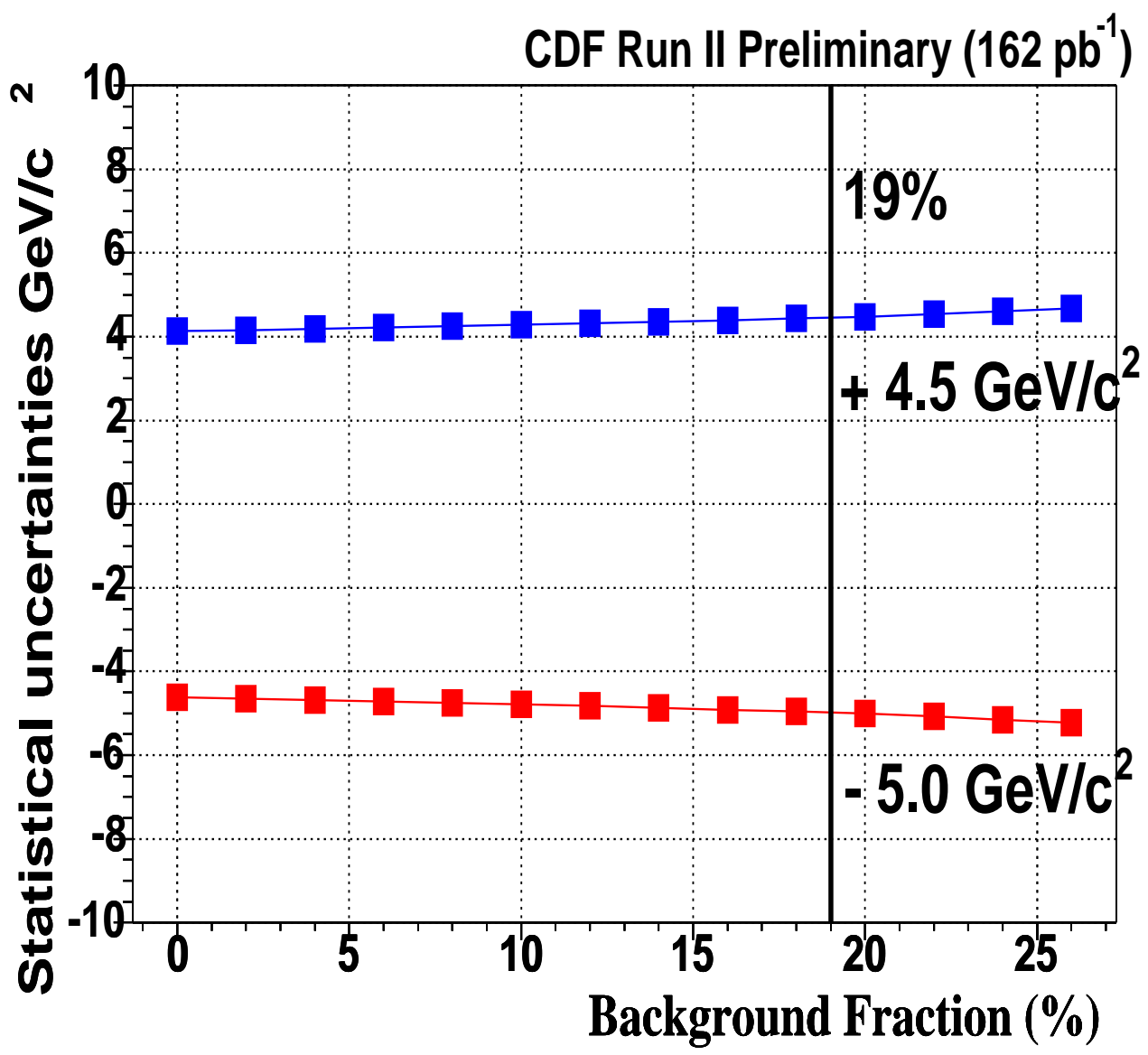

Figure 7.3: Estimated statistical uncertainties on top quark mass as a function of the background fraction. 


\subsection{The Statistical Uncertainty}

We check that the statistical uncertainty seen in the data is consistent with expectations for our measured mass. Figure 7.4 shows the expected negative and positive statistical uncertainties distribution for an input top mass of $175 \mathrm{GeV} / \mathrm{c}^{2}$, where the black arrows indicate the statistical uncertainties in the data. Each output is a results of pseudo experiment where a total number of events are fixed to be twenty-two, and contains background of 4.2 events which is Poisson fluctuated using expected numbers of events in table 5.6. The means of expectations are +5.4 , $-5.0 \mathrm{GeV} / \mathrm{c}^{2}\left(\mathrm{MPV}:+4.5,-4.1 \mathrm{GeV} / \mathrm{c}^{2}\right)$, where the data gives $+4.5,-5.0 \mathrm{GeV} / \mathrm{c}^{2}$.

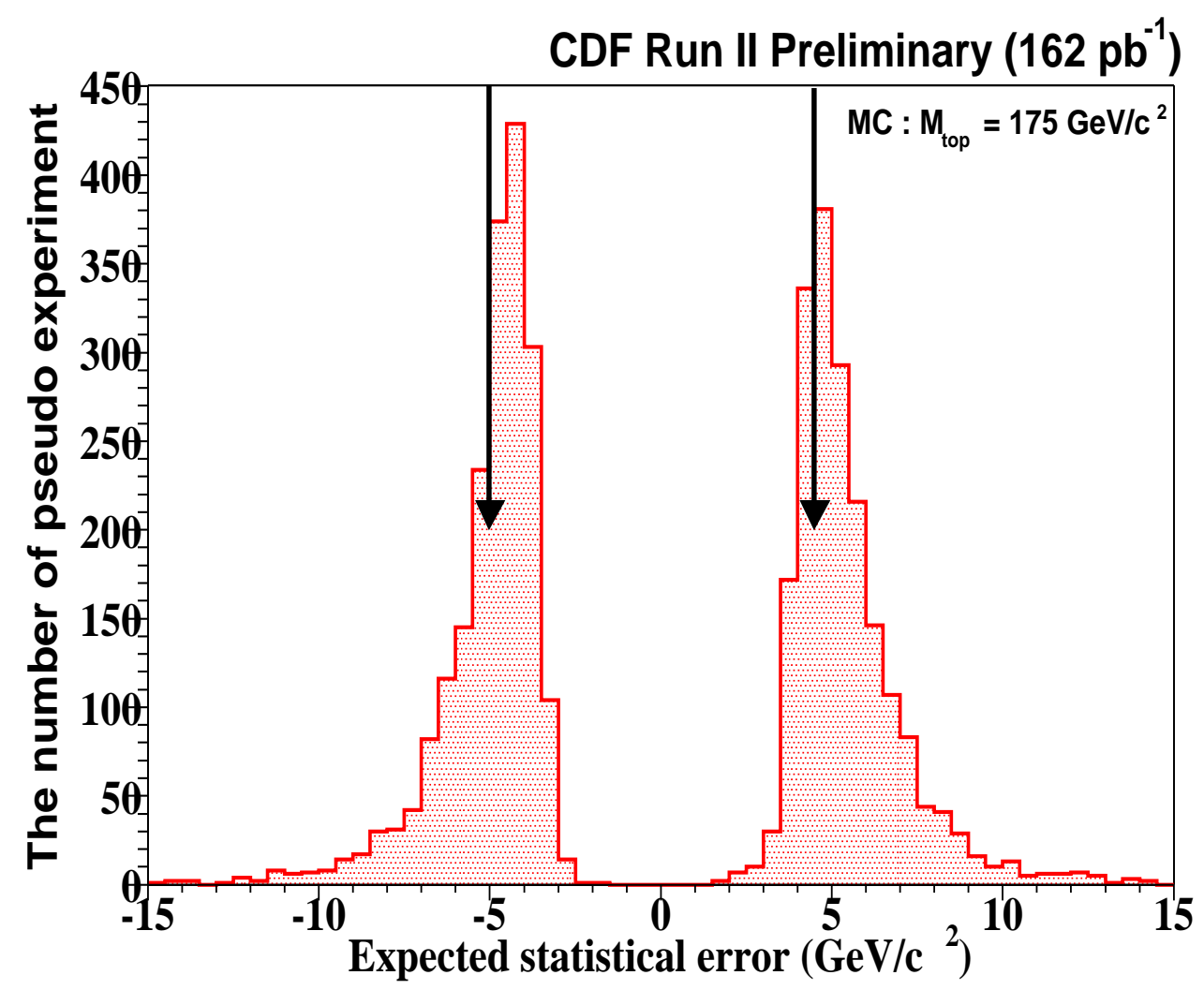

Figure 7.4: The expected positive and negative statistical uncertainties from 22-event pseudoexperiments from Monte Carlo events. An input top quark mass of $175 \mathrm{GeV} / \mathrm{c}^{2}$ is used for the comparison. The black arrows indicate those in the data. 


\section{Chapter 8}

\section{Systematic Uncertainty}

Systematic uncertainties arise whenever the simulated events may not accurately model the data. In this section, we have performed systematics studies with respect to known sources. For a given source of uncertainty, we make a change in the input sample and estimate the impact on the reconstructed top quark mass based on a number of pseudo experiments using Monte Carlo simulations, in order to avoid any biases due to the particular set of events in the data, where a signal input top quark mass is set to be $175 \mathrm{GeV} / c^{2}$. The reconstructed mass from all input samples for various systematic sources is calculated by the same procedure as used on the data sample, i.e. the likelihood computations, an application of mapping function with expected fraction of background of $19 \%$. Nominal values to be used is the reconsructed mass from HERWIG with an input top quark mass of $175 \mathrm{GeV} / c^{2}$ which was shown in figure 6.13.

\subsection{Jet energy scale}

In terms of the jet energy corrections, we performed two steps to obtain parton momenta from $t \bar{t}$ decay, one is the generic corrections calibrated by the QCD process, and another is the transfer function for top specific corrections of $b$ and $W$ daughter jets. We evaluate these two corrections separately by shifting the jet correction factor with $\pm \sigma$, and take one half of reconstructed mass difference between the two. The details of the generic jet energy corrections are described in Section 3.2. There are significant uncertainties on many aspects of the measurement of jet energies. Some of those are in the form of uncertainties on the energy measurements themselves, some are uncertainties on the detector simulation, which is used to derive many corrections, and 
ultimately to extract the top quark mass. Still others are best understood as uncertainties on jet production and fragmentation models used in the generators. Each uncertainties on each correction level is briefly described below:

- Level 1 : This is based on the dijet balancing procedure. The uncertainties take into account residual spline-interpolation effects, biases in the dijet balancing procedure, especially in the Wall (Crack) region, variation of plug calorimeter response with them, differences seen in the $\gamma$-jet balancing when it is checked, and half to differences between the corrections for the data and simulation. Table 8.1 summarizes the uncertainty which depends on $\eta$ of the jets.

- Level 2 : The uncertainty of the correction for the central calorimeter stability is assigned to be $1 \%$ and this is only for the data.

- Level 3 : The difference between Run I and Run II is studied using the $\gamma$-jet balancing and is measured to be $5 \%$, out of which $>2 \%$ difference comes from the difference in the CEM scale definition and the difference in the material before the tracking volume. The remaining $3 \%$ is taken as a systematic uncertainty in the data.

- Level 4 : Since in this data taking period, multiple interaction effect is very small, uncertainty is negligible as well as a small scale factor of this correction. (less than $1 \%$ )

- Level 5 : This systematic uncertainty is due to uncertainties in the modeling of jet showering and hadronization, which are tested by varying relevant parameters in the generator. Since the calorimeter response is non-linear, different fragmentation can affect the overall jet energy scale. This uncertainty only accounts for variations that affect the energy inside the jet cone $(\mathrm{R}=0.4)$.

We apply $\pm \sigma$ shift factor to both signal and background events and make event selection cuts on shifted samples. The reconstructed masses are then calculated by the DLM analysis procedure. As an example, the distribution of reconstructed masses from the uncertainty of level 1 Monte Carlo simulation ( $\pm \sigma$ shifted) are shown in figure 8.1. We take the half the difference between the means of $\pm \sigma$ distributions. The total uncertainty is taken as the quadrature sum of all uncertainties and is found to be $\pm 5.3 \mathrm{GeV} / c^{2}$ as summarized in table 8.2.

As previously mentioned, in this analysis, we use only generic jet energy correction level up to 5 . Instead, the transfer function is used for $t \bar{t}$ specific events. In principle, the transfer 


\begin{tabular}{ccc}
\hline \hline$\eta$ range & data & Simulation \\
\hline$|\eta|<0.2$ & $3 \%$ & $1 \%$ \\
$0.2<|\eta|<0.6$ & $0.5 \%$ & $1 \%$ \\
$0.6<|\eta|<1.0$ & $2 \%$ & $1 \%$ \\
$1.0<|\eta|<1.4$ & $4 \%$ & $7 \%$ \\
$1.4<|\eta|<2.0$ & $2 \%$ & $6 \%$ \\
$|\eta|>2.0$ & $7 \%$ & $7 \%$ \\
\hline \hline
\end{tabular}

Table 8.1: Systematic uncertainties on the relative jet corrections (Level 1) for data and Monte Carlo simulation.

\begin{tabular}{l|c|c}
\hline \hline Level & Contents & $\Delta \mathrm{M}_{\text {top }} \mathrm{GeV} / \mathrm{c}^{2}$ \\
\hline Level 1(MC) & $\eta$ Dependent Calibration & $2.0 \pm 0.1$ \\
Level 1(Data) & $\eta$ Dependent Calibration & $1.6 \pm 0.1$ \\
Level 2(Data) & Calorimeter Stability & $1.0 \pm 0.1$ \\
Level 3(MC) & Raw Scale & $3.2 \pm 0.1$ \\
Level 3(Data) & Raw Scale & $2.4 \pm 0.1$ \\
Level 5 & Absolute Scale & $2.0 \pm 0.1$ \\
\hline
\end{tabular}

Table 8.2: The systematic uncertainties for jet energy corrections in individual levels. Two entries in Level 1 and 3 are due to the correction factor differences between Monte Carlo simulation (MC) and Data. 


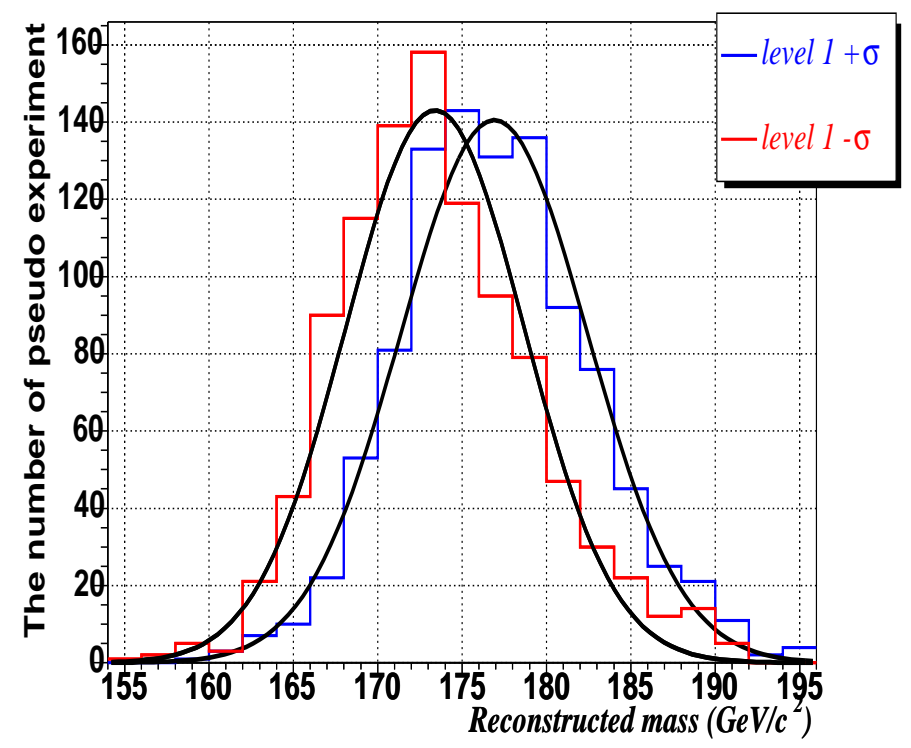

Figure 8.1: The systematic shift of the reconstructed mass from 1000 pseudo experiments due to jet energy correction (level 1: Relative correction) uncertainty. $+\sigma$ in blue, $-\sigma$ in red for the correction, where input top quark mass is $175 \mathrm{GeV} / c^{2}$.

functions should take uncertainties from the out of cone correction related to the fragmentation ambiguity, color flow, and the possible prediction of difference between the data and Monte Carlo simulations. Therefore, we use the uncertainties comes from out of cone correction corresponds to correction level 7. The uncertainty on the fraction of energy contained in the jet cone (also due to jet fragmentation modeling) is estimated in two parts, one between cone radius $R=0.4$ and 1.0 and the other between $R=1.0$ and $\infty$. We estimated an impact of this uncertainty on top quark mass to be $1.6 \mathrm{GeV} / c^{2}$. However, our assumption to extract the parton momenta strongly relies on Monte Carlo simulation, so we assign final uncertainty due to the transfer function to be $2.0 \mathrm{GeV} / \mathrm{c}^{2}$.

\subsection{Initial and final state hard radiation (ISR, FSR)}

For the initial state radiation effect on the mass, we have two samples which set the range of PYTHIA parameters using Drell-Yan $(e e, \mu \mu)$ data with lower $\Lambda_{Q C D}=100 \mathrm{MeV}$ and higher $\Lambda_{Q C D}=384 \mathrm{MeV}$ [63], where default value of $\Lambda_{Q C D}=192 \mathrm{MeV}$ which returned an uncertainty of $\pm 0.3 \pm 0.2 \mathrm{GeV} / c^{2}$. We have also tried to use PYTHIA tune A and tune B [40] which have 
different underlying energy tuning and found to be $\pm 0.2 \mathrm{GeV} / c^{2}$. We take statistically dominant value of $0.5 \mathrm{GeV} / c^{2}$ as a systematic error due to the initial state radiation. For the final state radiation, since ISR and FSR shower algorithms are the same, the same variations in $\Lambda_{Q C D}$ is used to generate FSR systematic samples. We estimated $0.5 \mathrm{GeV} / c^{2}$ as a systematic uncertainty due to FSR. For the comparison, we calculated the differences between FSR set ON and OFF. Of course, FSR off sample has no physics meaning at all, and provide an overestimated value of $1.6 \mathrm{GeV} / c^{2}$.

\subsection{Parton Distribution Function (PDF)}

In principle, the calculation of the top quark invariant mass is not affected by the choice of input PDF as long as we use the jets from final state $t \bar{t}$ partons. However we use PDF in our likelihood calculation and changing the PDF changes the size of ISR. Therefore, we need to estimate the uncertainty comes from PDF ambiguity. To do this, we added in quadrature uncertainties from three sources: the differences from the 20 pairs of CTEQ6M [42] uncertainty eigenvectors $( \pm \sigma)$, MRST using two different $\alpha_{s}$ values $\left(\Lambda_{Q C D}=228 \mathrm{MeV}\right.$ and $300 \mathrm{MeV}$ correspond to MRST72 and MRST 75), and the difference between the CTEQ5L [41] and MRST central values. The total uncertainty of PDF is estimated to be $2.0 \mathrm{GeV} / c^{2}$.

\subsection{Next-to-Leading Order uncertainty}

We are using leading order (LO) matrix element and CTEQ5L which is LO PDF for the likelihood so that next to leading order (NLO) effect has to be considered as a systematic error. We add together $q \bar{q}$ and $g g$ matrix element with PDF weight in the likelihood. Since we use CTEQ5L as a $\mathrm{PDF}$ in the likelihood computation, ratio between $q \bar{q}$ and $g g$ is not the one in data. LO(CTEQ5L) gives $6 \%$ for $g g$ and $94 \%$ for $q \bar{q}$, while NLO is $15 \%$ of $g g$ and $85 \%$ of $q \bar{q}$. We measured the mass 1) only $q \bar{q}, 2$ ) only $g g$ in the likelihood. The results are $172 \mathrm{GeV}$ for $g g$ only, and $176 \mathrm{GeV}$ for $q \bar{q}$ only. If the $g g$ fraction moves $\sim 10 \%$, we expect the error to be $\sim 0.4 \mathrm{GeV}$. Therefore, We take $0.4 \mathrm{GeV} / \mathrm{c}^{2}$ in this analysis. 


\subsection{Monte Carlo modeling}

\subsubsection{Jet energy resolution differences}

From the studies of $\gamma$-jet balance, it turned out that the CDF simulation underestimates the jet energy resolution by about $15 \%$. To see this effect, we performed 22 events pseudo experiments including the background of 4.2 events by an extra $15 \%$ of smearing. The top quark mass shift due to this extra smearing is found to be $-0.6 \mathrm{GeV} / c^{2}$. We take this as an uncertainty due to the jet energy resolution difference between data and Monte Carlo.

\subsubsection{Unclustered energy for $\boldsymbol{E}_{T}$}

We define "unclustered energy" as the calorimeter energy deposit which is not associated with jets that have Et $>8 \mathrm{GeV}$ and $|\eta|<2.5$. Even though jet energy scale is validated well by lots of works and each scale uncertainties is taken into account, unclustered energy can still contribute to missing Et so that the top quark mass may be affected by this differences between data and Monte Carlo. The top quark mass shift due to this effect is estimated to be $0.1 \mathrm{GeV} / c^{2}$ by adding up $1.0 \mathrm{GeV} / c^{2}$ and taking into account resolution discrepancy between MC and Data by a factor of 1.1 for unclustered energy contribution to missing Et. The factors of $1.0 \mathrm{GeV} / c^{2}$ and 1.1 resolution scale were determined by comparing data and Monte Carlo in each jet bin up to 4 jets.

\subsection{Background modeling and fraction}

To get the correct top quark mass, we apply the mapping function with the background fraction of $19 \%$ out of 22 events. We take into account the uncertainties of the fraction with $\pm 5 \%$. This gives $0.5 \mathrm{GeV} / c^{2}$ differences for top quark mass. Both estimates from using Monte Carlo and directly data give the same error of $0.5 \mathrm{GeV} / c^{2}$ for this uncertainty. And also background modeling uncertainty is $0.5 \mathrm{GeV} / c^{2}$ taken from the largest discrepancy in figure 6.10 at $19 \%$ background to take into account the difference of the size of mass-pulling in each background.

\subsection{Monte Carlo generators}

Monte Carlo generator must be considered as one of sources for the systematic uncertainties. We compare PYTHIA and HERWIG, HERWIG deals with spin correlations in the production and 
decays of $t \bar{t}$, while PYTHIA does not, although this should not affect the mass determination. The main difference between the two is in the fragmentation model, where PYTHIA uses the string model while HERWIG adopts the cluster model. We estimated it to be $0.6 \mathrm{GeV} / c^{2}$.

\subsection{Summary of systematic uncertainties}

The systematic uncertainties are summarized in Table 8.3. The largest one comes from the uncertainty in the jet energy measurement. The sum in quadrature of all the systematic uncertainties is $6.2 \mathrm{GeV} / \mathrm{c}^{2}$.

\begin{tabular}{cc}
\hline \hline Source & $\Delta \mathrm{M}_{\text {top }} \mathrm{GeV} / \mathrm{c}^{2}$ \\
\hline Jet Energy Corrections & 5.3 \\
ISR & 0.5 \\
FSR & 0.5 \\
PDFs & 2.0 \\
Generator & 0.6 \\
Spin correlation & 0.4 \\
NLO effect & 0.4 \\
Transfer Function & 2.0 \\
Background fraction( $\pm 5 \%)$ & 0.5 \\
Background modeling & 0.5 \\
Monte Carlo modeling & 0.6 \\
\hline Total & 6.2 \\
\hline \hline
\end{tabular}

Table 8.3: The summary of systematic uncertainties 


\section{Chapter 9}

\section{Cross Checks}

\subsection{Event by event comparisons}

It is good to look at other outputs of DLM and investigate any unexpected discrepancy between the data and Monte Carlo expectations. To do this, we compare the data and Monte Carlo for (1) the absolute value of the likelihood and (2) the maximum likelihood mass. The signal likelihood for the $i$-th event is defined as

$$
\boldsymbol{L}_{\text {event }}^{i}=\int L^{i}(M) d M
$$

where the integration is over the search region $[155-195] \mathrm{GeV} / \mathrm{c}^{2}$. We compare the log of the event likelihoods in the data and Monte Carlo samples in figure 9.1. The expectation values for the simulation sample are normalized to the numbers in the data (17.8 events for signal, and combined background of 4.2 events). Even though an absolute value itself does not have any physics meaning in DLM, by directly comparing the data with Monte Carlo expectations, Our calculation can be validated. As one can clearly see, the data is well understood by expectations.

Another check uses the event-by-event maximum likelihood mass. We use the maximum likelihood mass in each event which is shown in figure 9.2. Because the signal Monte Carlo sample was generated with $\mathrm{M}_{t o p}=175 \mathrm{GeV} / \mathrm{c}^{2}$, the data looks slightly higher than the Monte Carlo, as expected for a measured top quark mass of $177.8 \mathrm{GeV} / \mathrm{c}^{2}$. Note that the resolution of one event for signal top quark mass is found to be around $22 \mathrm{GeV} / \mathrm{c}^{2}$ from this study when a maximum likelihood mass is picked up. 


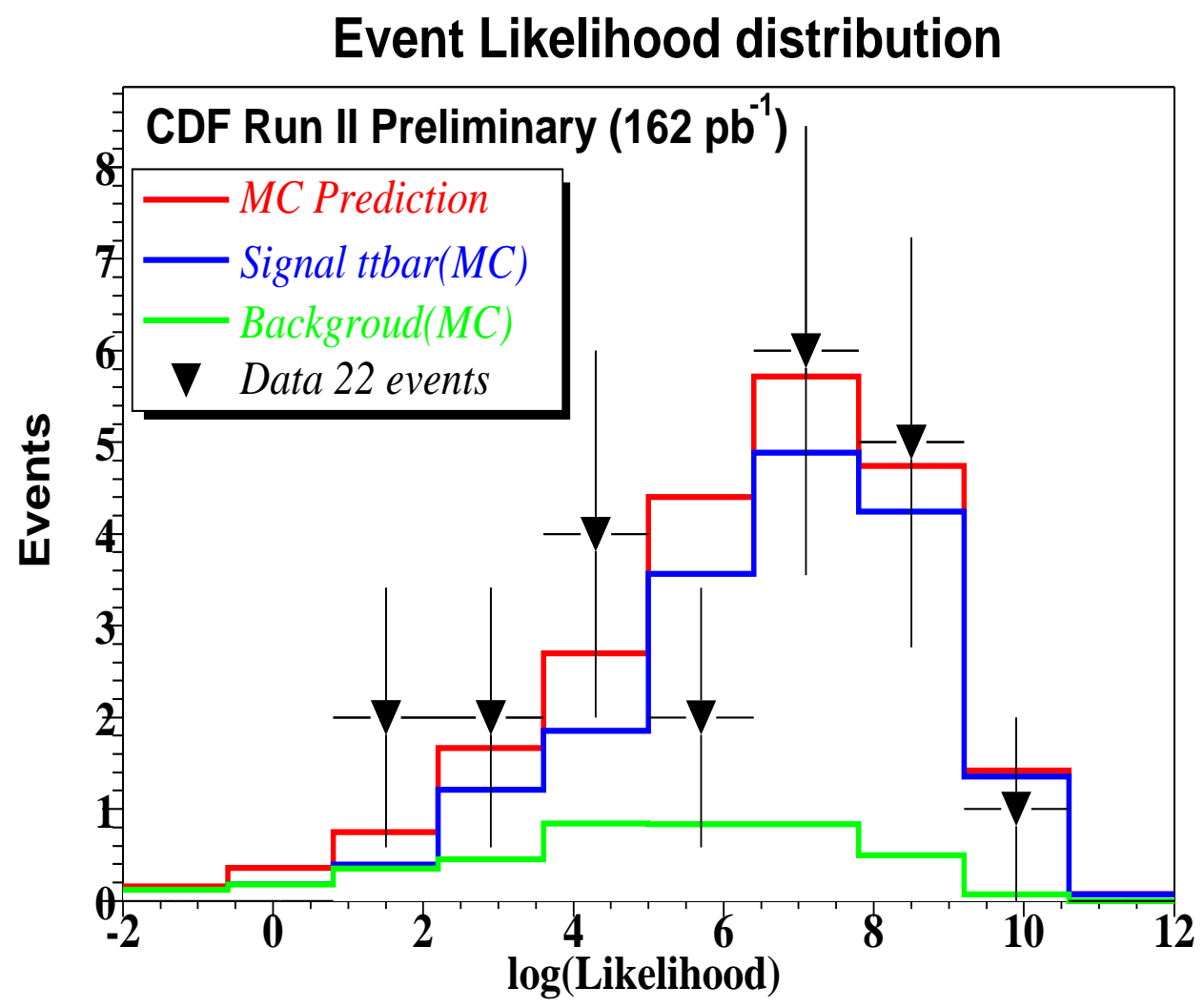

Figure 9.1: Event likelihood distribution. The number of signal and background events is normalized to the number of observed events (22 events). Blue is signal events and green is background, The red line corresponds to the combined Monte Carlo expectation. The triangles are the 22 data events. 


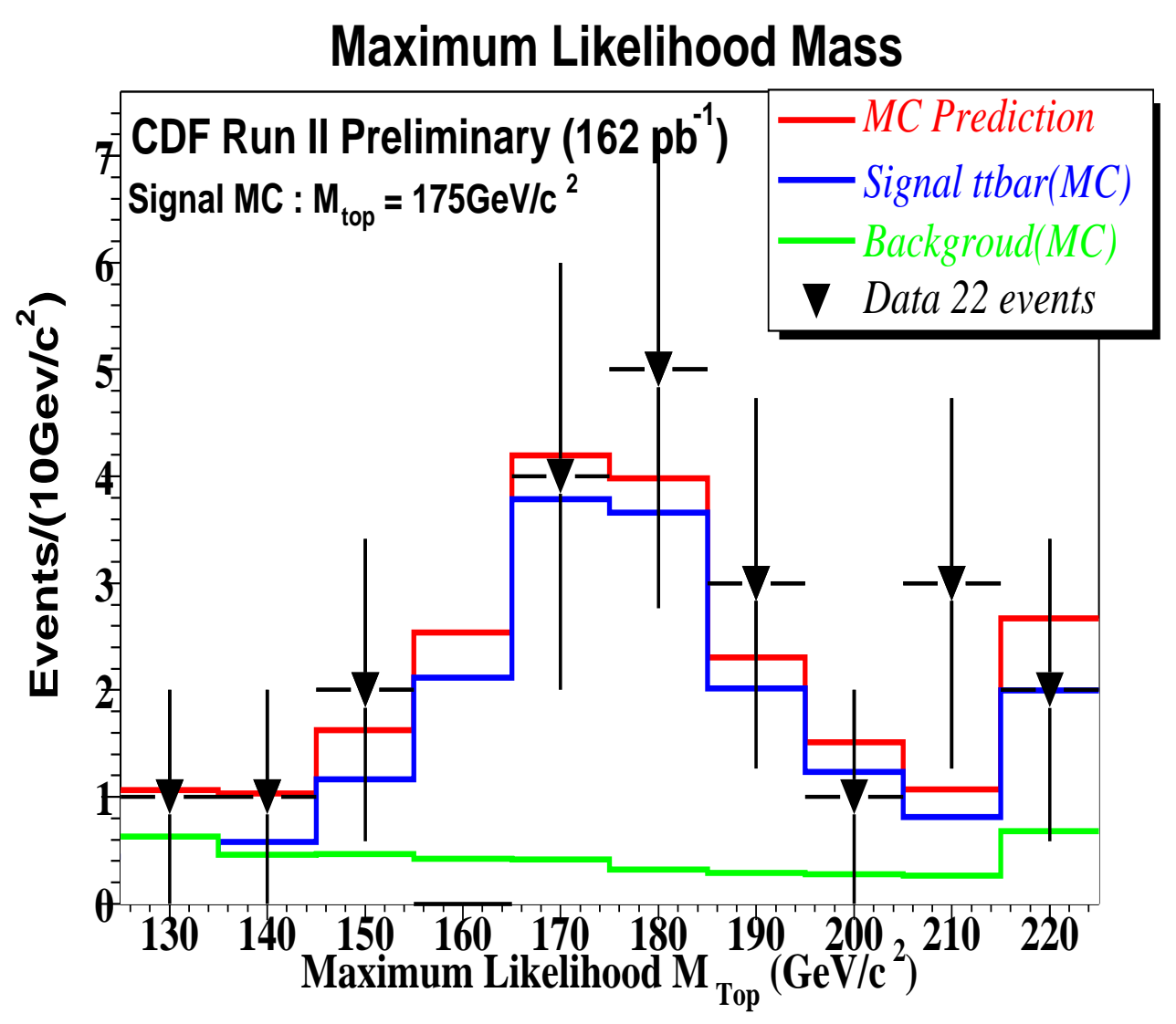

Figure 9.2: Maximum mass distribution in data and Monte Carlo. The signal Monte Carlo sample is for $\mathrm{M}_{t o p}=175$. The first(last) bin includes under(over)flow.Maximum mass distribution in data and Monte Carlo. The signal Monte Carlo sample is for $\mathrm{M}_{t o p}=175$. The first(last) bin includes under(over)flow 


\section{Chapter 10}

\section{Conclusions}

In conclusion, by using the dynamical likelihood method, we have measured the top quark mass to be $177.8_{-5.0}^{+4.5} \pm 6.2 \mathrm{GeV} / \mathrm{c}^{2}$ from 22 events corresponding to an integrated luminosity of $162 p b^{-1}$ accumulated by the CDF RunII experiment. Adding the uncertainties in quadrature gives a top mass of $177.8_{-8.0}^{+7.8} \mathrm{GeV} / \mathrm{c}^{2}$. Figure 10.1 shows the best results for each channel measured by $\mathrm{CDF}$ and $\mathrm{D} \varnothing$ experiments in RunII by far. The result of this analysis gave the smallest uncertainty of any of other analyses done in Run II so far. Furthermore, it is consistent with the world average which combined results in Run I. As previously described, a measurement of the top quark mass, along with a measurement of W boson mass, place limits on the Higgs boson. This result can contribute this expectation with other measurements.

For at least next three or four years, Fermilab will be still only accelerator in the world that will be able to produce top quarks. The CDF will keep taking data and collect at least $2-4 \mathrm{fb}^{-1}$. With this new data, it is hoped to measure the top quark mass within a total uncertainty of $2 \mathrm{GeV} / \mathrm{c}^{2}$. DLM is a very powerful technique not only for the mass measurement, but also to determine other properties and to probe even new physics beyond the standard model. This result supports the validity of this method, and it will provide us with further studies in particle physics field. 


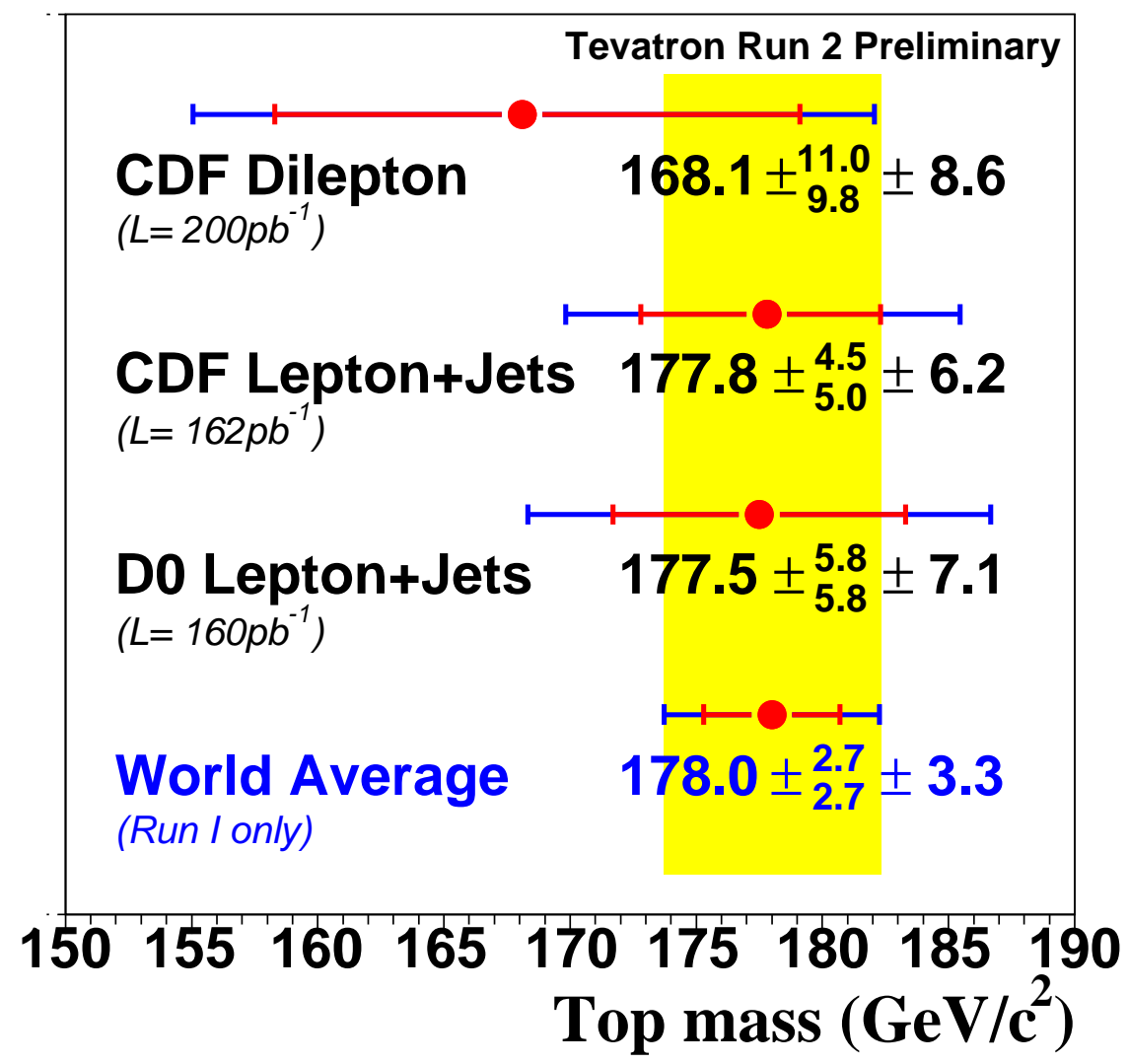

Figure 10.1: Summary of the best top quark mass measurements obtained from RunII Tevatron experiments so far. The result from DLM is referred as CDF Lepton+jets which is the best of all, and consistent with the world average extracted by an combination of all Run I results (blue bottom). 


\section{Bibliography}

[1] D. Gross, F. Wilczek, Asymptotically Free Gauge Theory, Phys. Rev. Lett. 30, 1343 (1973)

[2] M. Gell-Mann, A Schematic Model of Baryons and Mesons, Phys. Lett. 8, 214-215 (1964)

[3] R. P. Feynman, QED. The Strange Theory of Light and Matter, Princeton Science Library $(1985)$

[4] F. Halzen, A. Martin Quarks and Leptons, John Wiley and Sons (1984)

[5] R. K. Ellis, W. J. Stirling, B. R. Webber QCD and Collider Physics, Cambridge Monographs on Particle Physics, Nuclear Physics and Cosmology (1996)

[6] http://www-ed.fnal.gov/projects/exhibits/searching/

[7] L. M. Lederman The Discovery of the Upsilon, Bottom Quark, and B Mesons, 3rd International Symposium on the History of Particle Physics (1992)

[8] F. Abe et al. [CDF Collaboration], Phys. Rev. Lett. 73, 225 (1994); Phys. Rev. D50, 2966 (1994); S. Abachi et al., Phys. Rev. Lett. 74 (1995) 2632.

[9] M. Cacciari et al., JHEP 404 (2004) 68.

[10] S. Baroiant et al., Proceedings 4th International Workshop on Very High Multiplicity Physics, Alushta, Crimea, Ukraine, May (2003) FERMILAB-CONF-03/322-E

[11] F. Abe et al., Phys. Rev. Lett. 82 (1999) 2808.

[12] B. Abbott et al., Phys. Rev. D60 (1999) 052001.

[13] T. Affolder et al., Phys. Rev. D60 (2001) 032003.

[14] B. Abbott et al., Phys. Rev. D58 (1998) 052001. 
[15] F. Abe et al., Phys. Rev. Lett. 79 (1997) 1992.

[16] V.M. Abazov et al., Nature 429 (2004) 638. ; J.C. Estrada Vigil, hep-ex/0302031

[17] Tevatron Elecroweak Working Group, hep-ex/0404010. http://tevewwg.fnal.gov/

[18] LEP Electroweak Working Group, Phys. Lett. B565 (2003) 61; Update August 2004: http://lepewwg.web.cern.ch/LEPEWWG.

[19] http://lephiggs.web.cern.ch/LEPHIGGS/www/Welcome.html

[20] J.F. de Troconiz and F. J. Yndurain, Phys. Rev. D65 (2002) 093002.

[21] F. Abe et al. [CDF Collaboration], Nucl. Instrum. Meth. A 271, 387 (1988) and FERMILAB-PUB-96/390-E.

[22] K. Hagiwara et al. [Particle Data Group Collaboration], Phys. Rev. D 66, 010001 (2002).

[23] A. Sill et al., Nucl. Instrum. and Methods A447 (2000) 1.

[24] T. Affolder et al., Nucl. Instrum. and Methods 526 (2004) 249.

[25] F. Bedeschi et al. [CDF Collaboration], Nucl. Instrum. Meth. A 268, 50 (1988).

[26] L. Balka et al. [CDF Collaboration], Nucl. Instrum. Meth. A 267, 272 (1988).

[27] S. Bertolucci et al. [CDF Collaboration], Nucl. Instrum. Meth. A 267, 301 (1988).

[28] K. Byrum et al., Nucl. Instrum. Meth. A 364, 144 (1995).

[29] F. Abe et al. [CDF Collaboration], Phys. Rev. Lett. 73, 2662 (1994) [Erratum-ibid. 74, $1891(1995)]$.

[30] K. Byrum et al. [CDF Collaboration], "The Cdf Forward Muon System," Nucl. Instrum. Meth. A 268, 46 (1988).

[31] G. Ascoli et al., "Cdf Central Muon Detector," Nucl. Instrum. Meth. A 268 (1988) 33.

[32] T. Dorigo et al., Nucl. Instrum. and Methods A461 (2001) 560.

[33] W. Wagner, H. Stadie, T. Arisawa, K. Ikado, K. Maeshima, H. Wenzel and G. Veramendi, FERMILAB-CONF-02-269-E, International Europhysics Conference on HighEnergy Physics (HEP 2001), Budapest, Hungary, 12-18 Jul 2001 
[34] B. Angelos et al. [CDF Collaboration], International Conference on Computing in HighEnergy Physics and Nuclear Physics (CHEP 2000), Padova, Italy, 7-11 Feb 2000

[35] P. Canal et al., IEEE Trans. Nucl. Sci. 47 (2000) 240.

[36] F. Abe et al., Phys. Rev. D45 (1992) 1448.

[37] T. Affolder et al., Phys. Rev. D64 (2001) 032002.

[38] D. Acosta et al., submitted to Phys. Rev. D (2004).

[39] G. Marchesini et al., Comp. Phys. Commun. 67 (1992) 465 ; G. Corcella et al., JHEP 0101 (2001) 010.

[40] T. Sjostrand et al., Comp. Phys. Commun. 135 (2001) 238.

[41] J. Pumplin et al., JHEP 0207 (2002) 012. hep-ph/0201195.

[42] D. Stump et al., JHEP 0210 (2003) 046, hep-ph/0303013.

[43] P. Avery, K. Read, and G. Trahern (1985) CLEO Report CSN-212 (unpublished)

[44] M. L. Mangano et al., J. High Energy Phys. JHEP 07 (2003) 001.

[45] R. Brun and F. Carminati, CERN Programming Library Long Writeup W5013 (1993).

[46] G. Grindhammer, M. Rudowicz, and S. Peters, Nucl. Instrum. and Methods A290 (1990) 469.

[47] D. Acosta et al., (Template Method in RunII) to be submitted to Phys. Rev. D (2005).

[48] J. Campbell and R. Ellis, Phys. Rev. D60 (1999) 113006

[49] B. Harris et al., Phys. Rev. D66 (2002) 054024

[50] Kinematic fit + b tag Xsec paper: D. Acosta et al., submitted to Phys. Rev. D (2004).

[51] Kinematic fit w/o b tag Xsec paper : D. Acosta et al., to be submitted to Phys. Rev. D (2004).

[52] The Top Averaging Group for the CDF and D0 collaborations, L. Demortier et al., FERMILAB-TM-2084 (1999) 
[53] R. Bonciani, S. Catani, M.L. Mangano, and P. Nason, Nucl. Phys. B 529, 424 (1998); hep-ex/0303085.

[54] D. Acosta et al., F. Abe et al., Phys. Rev. D45 (1992) 1448. Phys. Rev. Lett. 93 (2004) 142001.

[55] K. Kondo, J.Phys. Soc. Jpn. 57 (1988) 4126.

[56] K. Kondo, J.Phys. Soc. Jpn. 60 (1991) 836.

[57] K. Kondo, T. Chikamatsu, S.H.Kim: J.Phys. Soc. Jpn. 62 (1993) 1177.

[58] K. Kondo, RISE Technical Reprot 2005-5, Advanced Research Institute for Science and Engineering, Waseda University.

[59] R.H. Dalitz and G.R. Goldstein Phys. Rev. D45 (1992) 1531

[60] K. Hagiwara et al., : Phys.Rev. D66, (2002) 010001.

[61] T. Sjostrand, L. Lonnblad, S. Mrenna, P. Skand : hep-ph/0108264 (2002)

[62] Top Mass Working Group: http://fcdfwww.fnal.gov/internal/physics/top/run2mass/

[63] http://www-cdf.fnal.gov/internal/physics/top/ISR/ISR.html 


\section{Publication List}

\section{Published Papers}

1. D. Acosta et al. [CDF Collaboration], "Measurement of the $t \bar{t}$ Production Cross Section in $p \bar{p}$ Collisions at $\sqrt{s}=1.96 \mathrm{TeV}$ Using Dilepton Events," Phys. Rev. Lett. 93, 142001 (2004)

2. D. Acosta et al. [CDF Collaboration], "Search for Electroweak Single Top Quark Production in $p \bar{p}$ Collisions at $\sqrt{s}=1.96 \mathrm{TeV}, "$ Phys. Rev. D 71, 012005 (2005)

3. K. Yorita et al.(15 authors) "Making the Most of Aging Scintillator," Nucl. Instrum. Meth. A453, 245 (2000)

\section{Oral Reports}

1. K. Yorita, "Measurement of the top quark mass with Template Method and DLM in CDF RunII", The Division of Particle Physics (DPF), Riverside, California USA, August. 2004

2. K. Yorita, "Measurement of the top quark mass using DLM in CDF RunII", Fermilab Joint Experimental-Theoretical Seminar, Fermilab, Batavia, Illinois USA, June 2004

3. K. Yorita, "Measurement of the top quark mass using DLM in CDF RunII", American Physics Society (APS), Colorado USA, May 2004

4. K. Yorita and K. Kondo, " $t \bar{t}$ events in the lepton plus jets channel studies", The Physical Society of Japan (JPS), Touhoku Gakuin University , Sendai, March 2003

5. K. Yorita, K. Kondo and R. Tsuchiya, "Full Reconstruction of $t \bar{t}$ events using DLM in the lepton plus jets channel", The Physical Society of Japan (JPS), Ritsumei-Kan University, Kyoto, March 2002 
6. K. Yorita, K. Kondo, R. Tsuchiya and K. Ebina, "A Test of CP violation mechanism in $t \bar{t}$ pair production", The Physical Society of Japan (JPS), Okinawa International University, Okinawa, September 2001

\section{Poster Award}

1. K. Yorita, "Top Quark Mass Measurement by Dynamical Likelihood Method at CDF RunII" New Perspectives Conference, Fermi National Accelerator Laboratory, Batavia, Illinois USA 3-4 June 2004. George Michail Memorial Poster Award (Awarded Third Prize) 